\title{
Total Synthesis and Structural Elucidation of
}

\section{Azaspiracid-1. Construction of Key Building Blocks for Originally Proposed Structure.}

K. C. Nicolaou, * Petri M. Pihko, Federico Bernal, Michael O. Frederick, Wenyuan Qian, Noriaki Uesaka, Nicole Diedrichs, Jürgen Hinrichs, Theocharis V. Koftis, Eriketi Loizidou, Goran Petrovic, Manuela Rodriquez, David Sarlah, Ning Zou.

Contribution from the Department of Chemistry and The Skaggs Institute for Chemical Biology, The Scripps Research Institute, 10550 North Torrey Pines Road, La Jolla, California 92037, and Department of Chemistry and Biochemistry, University of California, San Diego, 9500 Gilman Drive, La Jolla, California 92093.

Corresponding author e-mail: kcn@scripps.edu

\section{Supporting Information}

\section{Experimental Data for Compounds}

General Procedures. All reactions were carried out under an argon atmosphere with dry solvents under anhydrous conditions, unless otherwise noted. Dry tetrahydrofuran (THF), toluene, benzene, diethyl ether ( $\left.\mathrm{Et}_{2} \mathrm{O}\right)$, ethylene glycol dimethyl ether (DME), and methylene chloride $\left(\mathrm{CH}_{2} \mathrm{Cl}_{2}\right)$ were obtained by 
passing commercially available pre-dried, oxygen-free formulations through activated alumina columns. Yields refer to chromatographically and spectroscopically $\left({ }^{1} \mathrm{H}\right.$ NMR) homogeneous materials, unless otherwise stated. Reagents were purchased at the highest commercial quality and used without further purification, unless otherwise stated. Reactions were monitored by thin-layer chromatography (TLC) carried out on $0.25 \mathrm{~mm}$ E. Merck silica gel plates (60F-254) using UV light as visualizing agent and an ethanolic solution of phosphomolybdic acid and cerium sulfate, and heat as developing agents. E. Merck silica gel (60, particle size $0.040-0.063 \mathrm{~mm}$ ) was used for flash column chromatography. Preparative thin-layer chromatography (PTLC) separations were carried out on 0.25 or $0.50 \mathrm{~mm}$ E. Merck silica gel plates (60F-254). NMR spectra were recorded on Bruker DRX-600, DRX-500, AMX-500 or AMX-400 instruments and calibrated using residual undeuterated solvent as an internal reference. The following abbreviations were used to explain the multiplicities: $\mathrm{s}=$ singlet, $\mathrm{d}=$ doublet, $\mathrm{t}=$ triplet, $\mathrm{q}=$ quartet, $\mathrm{m}=$ multiplet, quin $=$ quintuplet, sext $=$ sextet, sep $=$ septet, br $=$ broad. IR spectra were recorded on a Perkin-Elmer 1600 series FT-IR spectrometer. Electrospray ionization (ESI) mass spectrometry (MS) experiments were performed on an API 100 Perkin Elmer SCIEX single quadrupole mass spectrometer at $4000 \mathrm{~V}$ emitter voltage. High-resolution mass spectra (HRMS) were recorded on a VG ZAB-ZSE mass spectrometer using MALDI (matrix-assisted laser-desorption ionization) or ESI (electrospray ionization).

Methyl phosphonate 28. To a solution of dimethyl methylphosphonate (77.1 g, $620 \mathrm{mmol}, 1.5$ equiv) in THF (1.0 L) at $-78{ }^{\circ} \mathrm{C}$ was added $n$-BuLi (388 mL, $1.6 \mathrm{M}$ solution in hexanes, $620 \mathrm{mmol}$, 1.5 equiv)

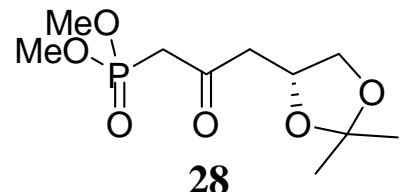

28 dropwise over $10 \mathrm{~min}$. The solution was stirred at $-78^{\circ} \mathrm{C}$ for $1 \mathrm{~h}$, followed by the dropwise addition of a solution of methyl ester $27^{1}$ (72.2 g, $414 \mathrm{mmol}, 1.0$ equiv) in THF (300 mL) over $10 \mathrm{~min}$. The reaction mixture was stirred at -78

${ }^{\circ} \mathrm{C}$ for $1 \mathrm{~h}$ and then quenched with saturated aqueous $\mathrm{NH}_{4} \mathrm{Cl}(500 \mathrm{~mL})$. The organic phase was separated and the aqueous layer was extracted with EtOAc $(3 \times 500 \mathrm{~mL})$. The combined organic layers were dried $\left(\mathrm{MgSO}_{4}\right)$, concentrated and then purified by flash column chromatography (silica gel, EtOAc:hexanes 1:1 $\rightarrow$ 9:1) to give methyl phosphonate $28(92.6 \mathrm{~g}, 348 \mathrm{mmol}, 84 \%)$ as a colorless oil. 28: $R_{\mathrm{f}}=0.35$ 
(silica gel, EtOAc:MeOH 95:5); $[\alpha]_{\mathrm{D}}{ }^{25}=-9.5\left(\mathrm{CHCl}_{3}, c=1.20\right)$; IR (film) $v_{\max }=2959,1716,1654$, 1288, 1032, $828 \mathrm{~cm}^{-1}$; ${ }^{1} \mathrm{H}$ NMR (400 MHz, $\mathrm{CDCl}_{3}$ ): $\delta=4.46$ (quin, $J=6.5 \mathrm{~Hz}, 1 \mathrm{H}$ ), 4.16 (dd, $J=8.5$, $6.2 \mathrm{~Hz}, 1 \mathrm{H}$ ), 3.79 (s, 3 H), 3.77 (s, 3 H), 3.55 (dd, $J=8.2,6.4 \mathrm{~Hz}, 1 \mathrm{H}$ ), 3.15 (dd, $J=5.2,1.1 \mathrm{~Hz}, 1 \mathrm{H}$ ), 3.11 (dd, $J=5.2,1.1 \mathrm{~Hz}, 1 \mathrm{H}$ ), 3.04 (dd, $J=17.3,6.4 \mathrm{~Hz}, 1 \mathrm{H}$ ), 2.79 (dd, $J=17.3,6.8 \mathrm{~Hz}, 1 \mathrm{H}$ ), 1.40 (s, $3 \mathrm{H}), 1.34$ (s, $3 \mathrm{H}) ;{ }^{13} \mathrm{C}$ NMR (100 MHz, $\left.\mathrm{CDCl}_{3}\right): \delta=199.4,109.3$, 71.6, 69.4, 53.6, 52.4, 48.4, 42.7, 26.5, 25.1; HR-MS [Matrix-assisted laser desorption / ionization (MALDI)]: calcd for $\mathrm{C}_{10} \mathrm{H}_{19} \mathrm{O}_{6} \mathrm{PNa}^{+}$ $\left[\mathrm{M}+\mathrm{Na}^{+}\right]:$289.0811, found 289.0808.

Enone 30. To a solution of methyl phosphonate 28 (73.5 g, 276 mmol, 1.3 equiv) in MeCN (500 mL) at $25^{\circ} \mathrm{C}$ was added $\mathrm{LiCl}(11.0 \mathrm{~g}, 276 \mathrm{mmol}, 1.3$ equiv), followed by $N, N$-diisopropylethylamine (37.8 mL,<smiles>CC(C=CC(=O)CCC1OC(C)(C)O1)COCc1ccccc1</smiles>
$217 \mathrm{mmol}, 1.0$ equiv). The solution was stirred at $25{ }^{\circ} \mathrm{C}$ for $2 \mathrm{~h}$, and then cooled to $0{ }^{\circ} \mathrm{C}$, at which time a solution of aldehyde $2 \mathbf{9}^{2}$ (38.7 g, $217 \mathrm{mmol}, 1.0$ equiv) in $\mathrm{MeCN}(100 \mathrm{~mL})$ was added dropwise over $20 \mathrm{~min}$. The reaction mixture was then stirred at $25{ }^{\circ} \mathrm{C}$ for $15 \mathrm{~h}$, and then quenched with saturated aqueous $\mathrm{NaCl}(300 \mathrm{~mL})$. The biphasic mixture was extracted with EtOAc $(4 \times 300 \mathrm{~mL})$ and the combined organic layers were dried $\left(\mathrm{MgSO}_{4}\right)$, concentrated, and purified by flash column chromatography (silica gel, 8:2 hexanes:EtOAc) to give enone 30 (59.4 g, $187 \mathrm{mmol}, 86 \%$ ) as a colorless oil. 30: $R_{\mathrm{f}}=0.37$ (silica gel, hexanes:acetone 8:2); $[\alpha]_{\mathrm{D}}{ }^{25}=-30.6\left(\mathrm{CHCl}_{3}, c=2.80\right)$; IR (film) $v_{\max }=2983,2871,1669,1369,1211,1057,698 \mathrm{~cm}^{-1} ;{ }^{1} \mathrm{H}$ NMR (500 MHz, $\mathrm{CDCl}_{3}$ ): $\delta=7.36-7.27$ (m, $5 \mathrm{H}$ ), 6.83 (dd, $J=16.0,7.0 \mathrm{~Hz}, 1 \mathrm{H}$ ), 6.12 (dd, $J=16.0$, $1.0 \mathrm{~Hz}, 1 \mathrm{H}$ ), 4.51 (s, $2 \mathrm{H}$ ), 4.50 (m, 1 H), 4.21 (dd, $J=8.0,6.0 \mathrm{~Hz}, 1 \mathrm{H}$ ), 3.56 (dd, $J=8.5,6.0 \mathrm{~Hz}, 1 \mathrm{H}$ ), 3.40 (dd, $J=6.5,2.0 \mathrm{~Hz}, 2$ H), 3.10 (dd, $J=17.0,5.0 \mathrm{~Hz}, 1 \mathrm{H}$ ), 2.70 (dd, $J=17.0,8.0 \mathrm{~Hz}, 1 \mathrm{H}$ ), 2.67 (m, $1 \mathrm{H}), 1.41$ (s, $3 \mathrm{H}), 1.36$ (s, $3 \mathrm{H}), 1.09$ (d, $J=7.0 \mathrm{~Hz}, 3 \mathrm{H}) ;{ }^{13} \mathrm{C}$ NMR $\left(125 \mathrm{MHz}, \mathrm{CDCl}_{3}\right.$ ): $\delta=197.9$, 150.5, 138.0, 129.7, 128.4, 127.7, 127.6, 108.6, 73.8, 73.1, 71.9, 69.6, 44.2, 37.0, 26.9, 25.4, 16.0; HRMS [Matrix-assisted laser desorption / ionization (MALDI)]: calcd for $\mathrm{C}_{19} \mathrm{H}_{26} \mathrm{O}_{4} \mathrm{Na}^{+}\left[\mathrm{M}+\mathrm{Na}^{+}\right]$: 341.1723, found 341.1720. 
Allylic alcohol 31. To a solution of enone 30 (21.0 g, $66.0 \mathrm{mmol}, 1.0$ equiv) in $\mathrm{Et}_{2} \mathrm{O}(1.1 \mathrm{~L})$ at $-40{ }^{\circ} \mathrm{C}$ was added LiI (70.7 g, $530 \mathrm{mmol}, 8.0$ equiv). The mixture was cooled to $-100{ }^{\circ} \mathrm{C}$ and $\mathrm{LiAlH}_{4}(25.1 \mathrm{~g}$, $\overbrace{\mathrm{OBn}}^{\mathrm{O}=}$ 660 mmol, 10 equiv) was added portionwise. The solution was stirred at -100 ${ }^{\circ} \mathrm{C}$ for $2 \mathrm{~h}$ and then warmed to $0{ }^{\circ} \mathrm{C}$. The reaction was quenched by the careful addition of $\mathrm{Na}_{2} \mathrm{SO}_{4} \cdot 10 \mathrm{H}_{2} \mathrm{O}$ (212 g, $660 \mathrm{mmol}, 10$ equiv). The resulting mixture was filtered through a short pad of silica gel, washed with EtOAc $(1.0 \mathrm{~L})$, dried $\left(\mathrm{MgSO}_{4}\right)$, and concentrated, and the resulting residue was purified by flash column chromatography (silica gel, EtOAc:hexanes 3:7) to give a single isomer of allylic alcohol 31 (20.7 g, $64.7 \mathrm{mmol}, 98 \%)$ as a colorless oil. 31: $R_{\mathrm{f}}=0.34$ (silica gel, EtOAc:hexanes 1:1); $[\alpha]_{\mathrm{D}}^{25}=-4.6\left(\mathrm{CHCl}_{3}, c=1.00\right)$; IR (film) $v_{\max }=$ 3441, 2983, 1665, 1453, 1369, 1063, $698 \mathrm{~cm}^{-1} ;{ }^{1} \mathrm{H}$ NMR (600 MHz, $\left.\mathrm{CDCl}_{3}\right): \delta=7.37-7.30(\mathrm{~m}, 5 \mathrm{H})$, 5.68 (dd, $J=15.4,7.0 \mathrm{~Hz}, 1 \mathrm{H}$ ), 5.52 (dd, $J=15.4,6.6 \mathrm{~Hz}, 1 \mathrm{H}), 4.50$ (s, $2 \mathrm{H}), 4.30$ (m, $1 \mathrm{H}), 4.24$ (dddd, $J=11.8,9.2,6.2,4.4$ Hz, $1 \mathrm{H}$ ), 4.08 (dd, $J=6.6,6.1 \mathrm{~Hz}, 1 \mathrm{H}), 3.56$ (t, $J=7.5 \mathrm{~Hz}, 1 \mathrm{H}$ ), 3.38 (dd, $J=9.2,6.6 \mathrm{~Hz}, 1 \mathrm{H}), 3.30$ (dd, $J=9.0,7.5 \mathrm{~Hz}, 1 \mathrm{H}), 2.78$ (br s, 1 H), 1.81-1.68 (m, $3 \mathrm{H}), 1.42$ (s, 3 H), 1.36 (s, $3 \mathrm{H}), 1.04$ (d, $J=7.0 \mathrm{~Hz}, 3 \mathrm{H}) ;{ }^{13} \mathrm{C}$ NMR (150 MHz, $\left.\mathrm{CDCl}_{3}\right): \delta=138.5,134.1,131.7,128.3$, 127.5, 127.4, 109.2, 75.03, 75.02, 72.9, 71.6, 69.7, 40.8, 36.4, 26.9, 25.7, 16.9; HR-MS [Matrix-assisted laser desorption / ionization (MALDI)]: calcd for $\mathrm{C}_{19} \mathrm{H}_{28} \mathrm{O}_{4} \mathrm{Na}^{+}\left[\mathrm{M}+\mathrm{Na}^{+}\right]$: 343.1880, found 343.1876.

Triol 32. Allylic alcohol 31 (60.9 g, 190 mmol) was dissolved in glacial acetic acid (315 mL) and $\mathrm{H}_{2} \mathrm{O}$ (157 mL) and heated at $40{ }^{\circ} \mathrm{C}$ for $5 \mathrm{~h}$. The reaction mixture was concentrated, azeotroped with toluene<smiles>C[C@@H](/C=C/[C@@H](O)C[C@H](O)CO)COCc1ccccc1</smiles>

32 $(4 \times 250 \mathrm{~mL})$, and the resulting residue was purified by flash column chromatography (silica gel, EtOAc:MeOH 95:5) to give triol 32 (51.7 g, 184 mmol, 97\%) as a colorless oil. 32: $R_{\mathrm{f}}=0.20$ (silica gel, EtOAc); $[\alpha]_{\mathrm{D}}{ }^{25}=-6.6$ $\left(\mathrm{CHCl}_{3}, c=0.40\right)$; IR (film) $v_{\max }=3381,2869,1454,1361,1091,698 \mathrm{~cm}^{-1} ;{ }^{1} \mathrm{H} \mathrm{NMR}(500 \mathrm{MHz}$, $\mathrm{CDCl}_{3}$ ): $\delta=7.37-7.30(\mathrm{~m}, 5 \mathrm{H}), 5.65$ (dd, $\left.J=15.8,7.0 \mathrm{~Hz}, 1 \mathrm{H}\right), 5.55$ (dd, $\left.J=15.8,6.8 \mathrm{~Hz}, 1 \mathrm{H}\right), 4.50$ (s, 2 H), 4.37 (ddd, $J=9.2,6.2,3.3 \mathrm{~Hz}, 1 \mathrm{H}$ ), 3.95 (ddd, $J=12.5,6.2,3.0 \mathrm{~Hz}, 1 \mathrm{H}$ ), 3.60 (dd, $J=11.0$, 3.7 Hz, $1 \mathrm{H}$ ), 3.47 (dd, $J=11.0,6.2 \mathrm{~Hz}, 1 \mathrm{H}$ ), 3.33 (ddd, $J=15.8,9.2,7.0 \mathrm{~Hz}, 2 \mathrm{H}$ ), 2.52 (quin, $J=7.0$ Hz, $1 \mathrm{H}), 1.72-1.65$ (m, $1 \mathrm{H}), 1.60$ (dt, $J=14.3,2.9 \mathrm{~Hz}, 1 \mathrm{H}), 1.01$ (d, $J=7.0 \mathrm{~Hz}, 3 \mathrm{H}) ;{ }^{13} \mathrm{C}$ NMR $(125$ 
$\left.\mathrm{MHz}, \mathrm{CDCl}_{3}\right): \delta=138.3,134.2,132.2,128.3,127.7,127.6,74.9,72.9,72.6,71.5,66.6,39.4,36.4,16.8 ;$

HR-MS [Matrix-assisted laser desorption / ionization (MALDI)]: calcd for $\mathrm{C}_{16} \mathrm{H}_{24} \mathrm{O}_{4} \mathrm{Na}^{+}\left[\mathrm{M}+\mathrm{Na}^{+}\right]$: 303.1567, found 303.1566.

Iodofuran 33. To a solution of triol 32 (46.8 g, $167 \mathrm{mmol}, 1.0$ equiv) in THF $(1.5 \mathrm{~L})$ at $0{ }^{\circ} \mathrm{C}$ was added $\mathrm{NaHCO}_{3}$ (140 g, 1.67 mol, 10 equiv) followed by $N$-iodosuccinimide (188 g, 835 mmol, 5.0 equiv). The

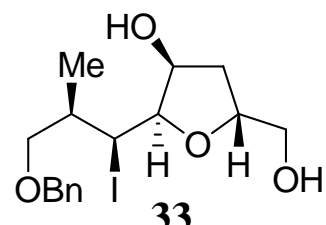
reaction mixture was stirred at $0{ }^{\circ} \mathrm{C}$ for $36 \mathrm{~h}$, then quenched with saturated aqueous $\mathrm{Na}_{2} \mathrm{~S}_{2} \mathrm{O}_{3}(1.0 \mathrm{~L})$, and $\mathrm{H}_{2} \mathrm{O}(500 \mathrm{~mL})$. The biphasic mixture was extracted with EtOAc $(4 \times 250 \mathrm{~mL})$ and the combined extracts were dried $\left(\mathrm{MgSO}_{4}\right)$, concentrated, and purified by flash column chromatography (silica gel, EtOAc:hexanes 1:9 $\rightarrow 1: 1$ ) to obtain a single isomer of iodofuran $33(59.9 \mathrm{~g}, 147 \mathrm{mmol}, 70 \%)$ as a colorless oil. 33: $R_{\mathrm{f}}=0.40$ (silica gel, EtOAc:hexanes 3:1); $[\alpha]_{\mathrm{D}}^{25}=+26.1\left(\mathrm{CHCl}_{3}, c=1.80\right)$; IR (film) $v_{\max }=3418,1652,1558,1456,1028$ $\mathrm{cm}^{-1}$; ${ }^{1} \mathrm{H}$ NMR (600 MHz, $\mathrm{CDCl}_{3}$ ): $\delta=7.35-7.28$ (m, $\left.5 \mathrm{H}\right), 4.65-4.62$ (m, $\left.2 \mathrm{H}\right), 4.53$ (s, 2 H), 4.45 (dddd, $J=12.3,9.4,5.3,2.9 \mathrm{~Hz}, 1 \mathrm{H}$ ), 4.20 (dd, $J=11.0,2.6 \mathrm{~Hz}, 1 \mathrm{H}$ ), 3.71 (ddd, $J=11.8,6.1,3.1 \mathrm{~Hz}$, $1 \mathrm{H}), 3.45$ (m, $1 \mathrm{H}), 3.34$ (dd, $J=9.2,5.7 \mathrm{~Hz}, 1 \mathrm{H}$ ), 3.24 (t, $J=9.2$ Hz, $1 \mathrm{H}), 2.05-2.00$ (m, 2 H), 1.67 (dddd, $J=12.0,8.8,6.5,2.4 \mathrm{~Hz}, 1 \mathrm{H}), 0.86(\mathrm{~d}, J=7.0 \mathrm{~Hz}, 3 \mathrm{H}) ;{ }^{13} \mathrm{C}$ NMR $\left(125 \mathrm{MHz}, \mathrm{CDCl}_{3}\right): \delta=$ 128.3, 127.7, 127.5, 120.7, 83.2, 79.7, 75.2, 74.6, 73.1, 64.7, 41.0, 35.7, 34.3, 13.8; HR-MS [Matrixassisted laser desorption / ionization (MALDI)]: calcd for $\mathrm{C}_{16} \mathrm{H}_{23} \mathrm{IO}_{4} \mathrm{Na}^{+}\left[\mathrm{M}+\mathrm{Na}^{+}\right]$: 429.0533, found 429.0534.

Silyl ether 34. Iodofuran 33 (58.7 g, $145 \mathrm{mmol}, 1.0$ equiv) was dissolved in $\mathrm{CH}_{2} \mathrm{Cl}_{2}(730 \mathrm{~mL})$ and cooled to $0{ }^{\circ} \mathrm{C}$. $\mathrm{Et}_{3} \mathrm{~N}$ (60.4 mL, $434 \mathrm{mmol}, 3.0$ equiv), and 4-DMAP (1.76 g, $14.4 \mathrm{mmol}, 0.1$ equiv) were

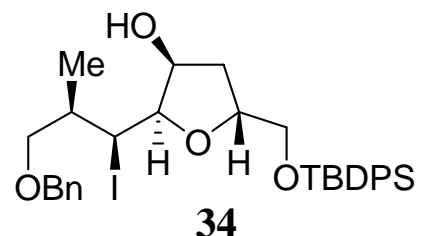
added, followed by the dropwise addition of TBDPSCl (52.6 mL, $202 \mathrm{mmol}$, 1.4 equiv). The solution was then allowed to warm to $25^{\circ} \mathrm{C}$ and stirred for 10 h. The reaction mixture was then quenched with saturated aqueous $\mathrm{NH}_{4} \mathrm{Cl}$ (400 mL) and the biphasic mixture was extracted with EtOAc $(4 \times 300 \mathrm{~mL})$. The combined organic layers were dried $\left(\mathrm{MgSO}_{4}\right)$, concentrated, and then purified by flash column chromatography (silica gel, 
hexanes:EtOAc 2:1) to give silyl ether $34(93.2 \mathrm{~g}, 144 \mathrm{mmol}, 99 \%)$ as a colorless oil. $\mathbf{3 4}: R_{\mathrm{f}}=0.28$ (silica gel, EtOAc:hexanes 1:4); $[\alpha]_{\mathrm{D}}{ }^{25}=+8.4\left(\mathrm{CHCl}_{3}, c=1.10\right)$; IR (film) $v_{\max }=3424,2931,1695$, 1460, 1109, $700 \mathrm{~cm}^{-1}$; ${ }^{1} \mathrm{H}$ NMR (600 MHz, $\mathrm{CDCl}_{3}$ ): $\delta=7.75-7.63$ (m, $\left.4 \mathrm{H}\right), 7.42-7.29$ (m, $11 \mathrm{H}$ ), $4.65-$ 4.63 (m, 2 H), 4.54 (s, 2 H), 4.45 (ddd, $J=10.0,7.2,3.8$ Hz, 1 H), 4.26 (dd, $J=11.4,2.6$ Hz, 1 H), 3.78 (dd, $J=11.0,3.5 \mathrm{~Hz}, 1 \mathrm{H}), 3.57$ (dd, $J=7.2,3.5 \mathrm{~Hz}, 1 \mathrm{H}$ ), 3.36 (dd, $J=9.2,5.7 \mathrm{~Hz}, 1 \mathrm{H}), 3.25$ (t, $J=$ 9.2 Hz, 1 H), 2.25 (ddd, $J=13.6,10.0,4.4$ Hz, 1 H), 2.05 (dd, $J=13.4,6.1$ Hz, 1 H), 1.76-1.72 (m, 2 H), 1.04 (s, $9 \mathrm{H}), 0.85$ (d, $J=7.0 \mathrm{~Hz}, 3 \mathrm{H}) ;{ }^{13} \mathrm{C}$ NMR (150 MHz, $\left.\mathrm{CDCl}_{3}\right): \delta=135.6,135.5,134.7$, 133.5, 129.7, 129.6, 128.3, 127.72, 127.71, 127.67, 127.61, 127.5, 83.4, 79.7, 75.2, 74.9, 73.0, 65.8, 41.5, 35.8, 34.3, 26.7, 19.2, 13.8; HR-MS [Matrix-assisted laser desorption / ionization (MALDI)]: calcd for $\mathrm{C}_{32} \mathrm{H}_{41} \mathrm{IO}_{4} \mathrm{SiNa}^{+}\left[\mathrm{M}+\mathrm{Na}^{+}\right]$: 667.1711, found 667.1714.

Bis-silyl ether 35. Silyl ether 34 (93.2 g, $145 \mathrm{mmol}$, 1.0 equiv) was dissolved in $\mathrm{CH}_{2} \mathrm{Cl}_{2}$ (765 mL), and cooled to $0{ }^{\circ} \mathrm{C}$. 2,6-Lutidine (67.3 mL, $578 \mathrm{mmol}, 4.0$ equiv) was added followed by the dropwise

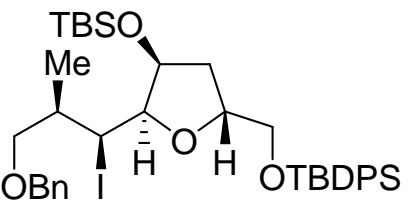

35 addition of TBSOTf (73.2 mL, $0.231 \mathrm{mmol}, 1.6$ equiv). The reaction mixture was stirred at $0{ }^{\circ} \mathrm{C}$ for $30 \mathrm{~min}$, and then quenched with saturated aqueous $\mathrm{NH}_{4} \mathrm{Cl}(400 \mathrm{~mL})$. The biphasic mixture was extracted with $\mathrm{CH}_{2} \mathrm{Cl}_{2}(3 \times 150$ $\mathrm{mL})$, then the combined organic layers were dried $\left(\mathrm{MgSO}_{4}\right)$, concentrated, and purified by flash column chromatography (silica gel, EtOAc:hexanes 2:8) to afford bis-silyl ether 35 (110 g, 145 mmol, 100\%) as a colorless oil. 35: $R_{\mathrm{f}}=0.36$ (silica gel, EtOAc:hexanes 1:19); $[\alpha]_{\mathrm{D}}{ }^{25}=+17.8\left(\mathrm{CHCl}_{3}, c=0.80\right)$; IR (film) $v_{\max }=3064,2930,1464,1253,1110,1053,704 \mathrm{~cm}^{-1} ;{ }^{1} \mathrm{H}$ NMR $\left(600 \mathrm{MHz}, \mathrm{CDCl}_{3}\right): \delta=7.75-$ 7.65 (m, 4 H), 7.36-7.28 (m, 11 H), 4.74 (dd, $J=11.0,2.2$ Hz, 1 H), 4.58 (br s, 1 H), 4.52 (s, 2 H), 4.38 (m, $1 \mathrm{H}$ ), 4.25 (dd, $J=10.5,1.7 \mathrm{~Hz}, 1 \mathrm{H}$ ), 3.79 (dd, $J=11.0,3.5 \mathrm{~Hz}, 1 \mathrm{H}$ ), 3.56 (dd, $J=11.0,3.5 \mathrm{~Hz}, 1$ H), 3.38 (dd, $J=9.2,5.7 \mathrm{~Hz}, 1 \mathrm{H}$ ), 3.28 (t, $J=9.2 \mathrm{~Hz}, 1 \mathrm{H}$ ), 2.13 (ddd, $J=13.6,9.5,3.5 \mathrm{~Hz}, 1 \mathrm{H}$ ), 1.89 (dd, $J=12.7,5.7 \mathrm{~Hz}, 1 \mathrm{H}), 1.75$ (m, 1 H), 1.27 (s, $9 \mathrm{H}), 1.04$ (s, $9 \mathrm{H}), 0.89$ (d, J = $7.0 \mathrm{~Hz}, 3 \mathrm{H}$ ), 0.21 (s, $3 \mathrm{H}), 0.13$ (s, $3 \mathrm{H}) ;{ }^{13} \mathrm{C}$ NMR (150 MHz, $\left.\mathrm{CDCl}_{3}\right): \delta=138.6,135.6,135.5,135.3,133.6,133.4,129.6$, 129.5, 128.2, 127.6, 127.5, 127.3, 84.6, 79.6, 75.7, 74.6, 73.0, 65.7, 42.1, 36.7, 34.2, 26.7, 25.9, 19.2, 
18.0, 13.9, -4.0, -4.4; HR-MS [Matrix-assisted laser desorption / ionization (MALDI)]: calcd for $\mathrm{C}_{38} \mathrm{H}_{55} \mathrm{IO}_{4} \mathrm{Si}_{2} \mathrm{Na}^{+}\left[\mathrm{M}+\mathrm{Na}^{+}\right]:$781.2576, found 781.2567.

Furan 36. Bis-silyl ether 35 (98.84 g, 130 mmol, 1.0 equiv) was dissolved in EtOH (1.0 L) at $25^{\circ} \mathrm{C}$ and purged with argon several times. Then a slurry of Raney-Nickel (250 g, 250\% w/w) pre-washed with

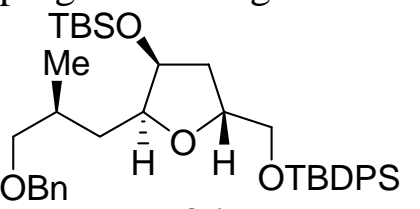

36

EtOH was added and the flask was purged several times with hydrogen. The reaction mixture was then allowed to stir under a hydrogen atmosphere for $1 \mathrm{~h}$. When the reaction was complete the slurry was filtered through a short pad of celite, and washed with EtOH $(4 \times 400 \mathrm{~mL})$. The filtrate was concentrated and purified by flash column chromatography (silica gel, EtOAc:hexanes 2:8) to yield furan 36 (81.6 g, $129 \mathrm{mmol}, 99 \%$ ) as a colorless oil. 36: $R_{\mathrm{f}}=0.34$ (silica gel, EtOAc:hexanes 1:19); $[\alpha]_{\mathrm{D}}{ }^{25}=+17.8\left(\mathrm{CHCl}_{3}, c=0.70\right)$; IR (film) $v_{\max }=2928,2855,1633,1470,1112,700 \mathrm{~cm}^{-1} ;{ }^{1} \mathrm{H}$ NMR (600 MHz, $\left.\mathrm{CDCl}_{3}\right): \delta=7.73-7.67$ (m, $\left.4 \mathrm{H}\right)$, 7.41-7.35 (m, 6 H), 7.33-7.30 (m, 5 H), 4.51 (d, $J=14.5$ Hz, 1 H), 4.48 (d, $J=14.5$ Hz, 1 H), 4.26 (br s, 1 H), 4.24-4.18 (m, 1 H), 3.99 (dt, $J=5.3,3.5$ Hz, 1 H), 3.77 (dd, $J=10.6,3.5$ Hz, $1 \mathrm{H}), 3.61$ (dd, $J=$ 11.0, $3.5 \mathrm{~Hz}, 1 \mathrm{H}$ ), 3.44 (dd, $J=9.2,5.2 \mathrm{~Hz}, 1 \mathrm{H}$ ), 3.28 (dd, $J=8.8,7.0 \mathrm{~Hz}, 1 \mathrm{H}$ ), 2.12 (ddd, $J=13.1$, 9.2, $4.8 \mathrm{~Hz}, 1 \mathrm{H}), 2.02$ (ddd, $J=19.8,12.5,5.9 \mathrm{~Hz}, 1 \mathrm{H}), 1.87$ (dd, $J=10.6,5.5 \mathrm{~Hz}, 1 \mathrm{H}), 1.74$ (ddd, $J=$ 14.0, 9.2, $5.2 \mathrm{~Hz}, 1 \mathrm{H}), 1.31$ (ddd, $J=13.6,8.8,4.0 \mathrm{~Hz}, 1 \mathrm{H}), 1.27$ (s, $9 \mathrm{H}), 1.25$ (d, $J=7.0 \mathrm{~Hz}, 3 \mathrm{H}$ ), 1.04 (s, $9 \mathrm{H}), 0.08$ (s, $3 \mathrm{H}), 0.07$ (s, $3 \mathrm{H}) ;{ }^{13} \mathrm{C}$ NMR (150 MHz, $\mathrm{CDCl}_{3}$ ): $\delta=138.9,135.6,135.5,133.7$, 133.6, 129.6, 129.5, 128.2, 127.6, 127.5, 127.4, 127.2, 80.8, 77.3, 76.4, 74.3, 72.8, 66.2, 37.6, 33.8, 30.7, 26.8, 25.8, 19.2, 18.1, 17.2, -4.4, -4.9; HR-MS [Matrix-assisted laser desorption / ionization (MALDI)]: calcd for $\mathrm{C}_{38} \mathrm{H}_{56} \mathrm{O}_{4} \mathrm{Si}_{2} \mathrm{Na}^{+}\left[\mathrm{M}+\mathrm{Na}^{+}\right]$: 655.3609, found 655.3602.

Primary alcohol 37. Furan 36 (76.8 g, $121 \mathrm{mmol}, 1.0$ equiv) was dissolved in EtOH (770 mL) at $25^{\circ} \mathrm{C}$ and purged with argon several times. Then 20\% $\mathrm{Pd}(\mathrm{OH})_{2} / \mathrm{C}(19.2 \mathrm{~g}, 25 \% \mathrm{w} / \mathrm{w}, 0.2$ equiv) was added and the flask was purged with hydrogen several times and the reaction mixture was stirred under an atmosphere of hydrogen at $25^{\circ} \mathrm{C}$ for $3 \mathrm{~h}$. The reaction mixture was then filtered through a short pad of celite and washed with EtOH (1.0 L), concentrated, and the resulting residue was purified by flash 
column chromatography (silica gel, EtOAc:hexanes 2:8) to afford primary alcohol 37 (58.0 g, 107 mmol, 88\%) as a colorless oil. 37: $R_{\mathrm{f}}=0.22$ (silica gel, EtOAc:hexanes 3:1); $[\alpha]_{\mathrm{D}}{ }^{25}=+5.5\left(\mathrm{CHCl}_{3}, c=\right.$ 1.00); IR (film) $v_{\max }=3447,2928,1472,1255,1112,834,702 \mathrm{~cm}^{-1}$; ${ }^{1} \mathrm{H}$ NMR
$\left(600 \mathrm{MHz}, \mathrm{CDCl}_{3}\right): \delta=7.70-7.65(\mathrm{~m}, 4 \mathrm{H}), 7.43-7.36(\mathrm{~m}, 6 \mathrm{H}), 4.33$ (dddd, $J$ 37

H), 3.73 (dd, $J=11.0,4.0 \mathrm{~Hz}, 1 \mathrm{H}$ ), 3.61 (dd, $J=11.0,4.0 \mathrm{~Hz}, 1 \mathrm{H}$ ), 3.57 (ddd, $J=11.4,7.4,3.3 \mathrm{~Hz}, 1 \mathrm{H}$ ), 3.48-3.40 (m, 1 H), 3.38 (br s, 1 H), 2.07 (ddd, $J$ = 13.1, 8.8, 4.4 Hz, 1 H), 1.87 (ddd, $J=12.7,6.1,1.3 \mathrm{~Hz}, 1 \mathrm{H}), 1.85-1.75$ (m, 1 H), 1.60 (ddd, $J=22.5,17.6,11.9 \mathrm{~Hz}, 1 \mathrm{H}$ ), 1.47 (ddd, $J$ = 14.9, 7.0, 2.2 Hz, 1 H), 1.04 (s, 9 H), 0.93 (d, $J=7.0$ Hz, 3 H), 0.90 (s, 9 H), 0.08 (s, 3 H), 0.07 (s, $3 \mathrm{H}) ;{ }^{13} \mathrm{C}$ NMR (150 MHz, $\left.\mathrm{CDCl}_{3}\right): \delta=135.6,135.5,133.5$, 133.3, 129.7, 129.6, 127.6, 127.5, 82.5, 77.8, 74.6, 68.6, 65.9, 37.5, 35.8, 35.1, 26.7, 25.7, 19.2, 18.6, 18.1, -4.5, -4.9; HR-MS [Matrixassisted laser desorption / ionization (MALDI)]: calcd for $\mathrm{C}_{31} \mathrm{H}_{50} \mathrm{O}_{4} \mathrm{Si}_{2} \mathrm{Na}^{+}\left[\mathrm{M}+\mathrm{Na}^{+}\right]$: 565.3140, found 565.3134.

Aldehyde 17. Primary alcohol 37 (58.0 g, $107 \mathrm{mmol}, 1.0$ equiv) was dissolved in $\mathrm{CH}_{2} \mathrm{Cl}_{2}(500 \mathrm{~mL})$ and cooled to $0{ }^{\circ} \mathrm{C}$. Dess-Martin periodinane $(90.7 \mathrm{~g}, 214 \mathrm{mmol}, 2.0$ equiv) was added portionwise and the

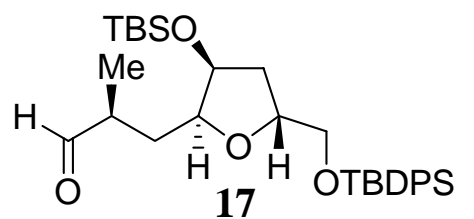
reaction mixture was allowed to warm to $25{ }^{\circ} \mathrm{C}$ over $2 \mathrm{~h}$. The reaction mixture was then quenched with saturated aqueous $\mathrm{Na}_{2} \mathrm{~S}_{2} \mathrm{O}_{3}(300 \mathrm{~mL})$ and extracted with $\mathrm{CH}_{2} \mathrm{Cl}_{2}(3 \times 250 \mathrm{~mL})$. The combined organic extracts were dried $\left(\mathrm{MgSO}_{4}\right)$, concentrated, and purified by flash column chromatography (silica gel, EtOAc:hexanes 3:7) to afford aldehyde 17 (44.85 g, $83.0 \mathrm{mmol}, 99 \%$ ) as a colorless oil. 17: $R_{\mathrm{f}}=0.66$ (silica gel, EtOAc:hexanes 2:8); $[\alpha]_{\mathrm{D}}^{25}=+13.9\left(\mathrm{CHCl}_{3}, c=1.20\right)$; IR (film) $v_{\max }=2931,2860,1724,1463,1255$, 1109, 833, 703, $502 \mathrm{~cm}^{-1}$; ${ }^{1} \mathrm{H}$ NMR (600 MHz, $\mathrm{CDCl}_{3}$ ): $\delta=9.67$ (d, $\left.J=2.1 \mathrm{~Hz}, 1 \mathrm{H}\right), 7.70-7.65$ (m, 4 H), 7.43-7.34 (m, 6 H), 4.31 (br s, 1 H), 4.28-4.25 (m, 1 H), 3.97 (dt, $J=9.7,3.8,1$ H), 3.76 (dd, $J=$ 11.0, $4.0 \mathrm{~Hz}, 1 \mathrm{H}$ ), 3.60 (dd, $J=11.0,4.0 \mathrm{~Hz}, 1 \mathrm{H}), 2.59-2.50$ (m, 1 H), 2.10-2.09 (m, 2 H), 1.89 (ddd, $J$ = 12.8, 6.7, $2.0 \mathrm{~Hz}, 1 \mathrm{H}$ ), 1.39 (ddd, $J=14.0,7.9,3.7 \mathrm{~Hz}, 1 \mathrm{H}$ ), 1.10 (d, $J=7.0 \mathrm{~Hz}, 3 \mathrm{H}$ ), 1.04 (s, $9 \mathrm{H}$ ), 0.90 (s, 9 H), 0.08 (s, $3 \mathrm{H}$ ), 0.07 (s, $3 \mathrm{H}) ;{ }^{13} \mathrm{C}$ NMR (150 MHz, $\left.\mathrm{CDCl}_{3}\right): \delta=205.2,135.6,135.5,129.62$, 
129.60, 129.0, 128.2, 127.64, 127.61, 80.3, 77.5, 73.9, 66.0, 43.9, 37.4, 31.0, 26.7, 25.7, 19.2, 18.1, 13.4 , -4.5, -4.9; HR-MS [Matrix-assisted laser desorption / ionization (MALDI)]: calcd for $\mathrm{C}_{31} \mathrm{H}_{48} \mathrm{O}_{4} \mathrm{Si}_{2} \mathrm{Na}^{+}$ $\left[\mathrm{M}+\mathrm{Na}^{+}\right]:$563.2983, found 563.2973.

Sulfoxide 19. To a solution of $i$ - $\operatorname{Pr}_{2} \mathrm{NEt}\left(0.24 \mathrm{~mL}, 1.67 \mathrm{mmol}, 1.2\right.$ equiv) in THF $(2 \mathrm{~mL})$ at $-78{ }^{\circ} \mathrm{C}$ was added $n$-BuLi (1.12 mL, 1.6 M solution in hexanes, $1.67 \mathrm{mmol}, 1.2$ equiv) and the resulting solution was

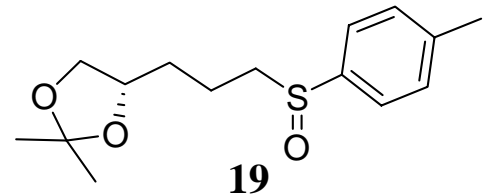
stirred for 30 min at $-78{ }^{\circ} \mathrm{C}$. A solution of $(R)-(+)$-methyl $p$-tolyl sulfoxide (216 mg, $1.4 \mathrm{mmol}, 1.0$ equiv) was added in THF ( $2 \mathrm{~mL}$ ) and the resulting mixture was stirred for an additional 10 min at which time iodide $\mathbf{3 8}^{3}$ (360 mg, $1.4 \mathrm{mmol}$, 1.0 equiv) was added in THF ( $1 \mathrm{~mL})$. The reaction mixture was stirred for an additional $30 \mathrm{~min}$ at $-78{ }^{\circ} \mathrm{C}$ and then quenched with saturated aqueous $\mathrm{NH}_{4} \mathrm{Cl}(3 \mathrm{~mL})$. The biphasic mixture was extracted with EtOAc $(3 \times 4 \mathrm{~mL})$, and the combined organic extracts were dried $\left(\mathrm{MgSO}_{4}\right)$, concentrated, and purified by flash column chromatography (silica gel, EtOAc:hexanes 3:1) to afford sulfoxide 19 (327 mg, $1.16 \mathrm{mmol}, 83 \%$ ) as a colorless oil. 19: $R_{f}=0.40$ (silica gel, EtOAc:hexanes 2:1); ${ }^{1} \mathrm{H}$ NMR $\left(400 \mathrm{MHz}, \mathrm{CDCl}_{3}\right) \delta=7.50$ (d, $\left.J=8.2 \mathrm{~Hz}, 2 \mathrm{H}\right), 7.32$ (d, $\left.J=8.0 \mathrm{~Hz}, 2 \mathrm{H}\right), 4.05-4.01$ (m, $\left.2 \mathrm{H}\right), 3.51$ (t, $J$ $=6.4 \mathrm{~Hz}, 1 \mathrm{H}), 2.83(\mathrm{t}, J=7.6 \mathrm{~Hz}, 1 \mathrm{H}), 2.82(\mathrm{t}, J=7.9 \mathrm{~Hz}, 1 \mathrm{H}), 2.41(\mathrm{~s}, 3 \mathrm{H}), 1.85-1.73(\mathrm{~m}, 2 \mathrm{H})$, 1.69-1.66 (m, $2 \mathrm{H}), 1.38$ (s, $3 \mathrm{H}), 1.32$ (s, $3 \mathrm{H}) ;{ }^{13} \mathrm{C}$ NMR (100 MHz, $\left.\mathrm{CDCl}_{3}\right) \delta=134.1,129.9,125.6$, 124.0, 75.4, 69.2, 57.1, 55.6, 32.4, 26.9, 25.6, 21.4, 18.9; HR-MS [Matrix-assisted laser desorption / ionization (MALDI)]: calcd for $\mathrm{C}_{15} \mathrm{H}_{22} \mathrm{O}_{3} \mathrm{SNa}^{+}\left[\mathrm{M}+\mathrm{Na}^{+}\right]$: 305.1187, found 305.1195.

Secondary alcohol 39. Magnesium turnings ( $12.2 \mathrm{~g}, 502 \mathrm{mmol}, 6.0$ equiv) were added to THF (500 $\mathrm{mL})$ in a flask equipped with a reflux condenser at $25{ }^{\circ} \mathrm{C}$. 4-bromo-1-butene $(42.4 \mathrm{~mL}, 418 \mathrm{mmol}, 5.0$

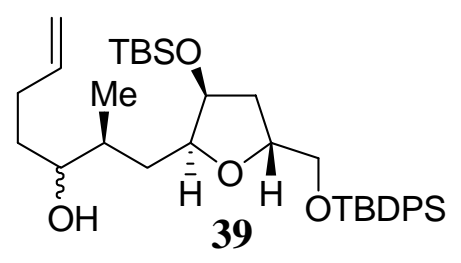
equiv) was added dropwise and the mixture was allowed to stir at $25{ }^{\circ} \mathrm{C}$ for 2 h. Aldehyde 17 (44.9 g, 83.0 mmol, 1.0 equiv) was dissolved in THF (280 $\mathrm{mL}$ ) and cooled to $-78{ }^{\circ} \mathrm{C}$. The freshly prepared Grignard reagent was then added dropwise via cannula to the aldehyde and the resulting mixture was stirred at $-78{ }^{\circ} \mathrm{C}$ for $30 \mathrm{~min}$, and then at $0{ }^{\circ} \mathrm{C}$ for $1.5 \mathrm{~h}$. The reaction mixture was then quenched with 
saturated aqueous $\mathrm{NH}_{4} \mathrm{Cl}(500 \mathrm{~mL})$, and extracted with EtOAc $(3 \times 300 \mathrm{~mL})$. The combined organic extracts were dried $\left(\mathrm{MgSO}_{4}\right)$, concentrated, and purified by flash column chromatography (silica gel, EtOAc:hexanes 1:9) to give a 1:1 ratio of secondary alcohols 39 (46.65 g, $78.1 \mathrm{mmol}, 93 \%$ ) as a colorless oil. 39 (mixture of epimers ca 1:1): $R_{\mathrm{f}}=0.54$ (silica gel, EtOAc:hexanes 2:8); IR (film) $v_{\max }$ $=3450,2928,2856,1471,1427,1255,1112,834,702 \mathrm{~cm}^{-1} ;{ }^{1} \mathrm{H}$ NMR $\left(600 \mathrm{MHz}, \mathrm{CDCl}_{3}\right): \delta=7.70-$ 7.65 (m, 4 H), 7.47-7.34 (m, 6 H), 5.84 (dddd, $J=19.7,12.7,6.1,2.6$ Hz, 1 H), 5.12-4.92 (m, 2 H), 4.35-4.31 (m, 1 H), 4.25 (br s, 1 H), 4.00-3.95 (m, 1 H), 3.73 (dd, $J=13.6,5.5$ Hz, 1 H), 3.67-3.58 (m, 2 H), 3.45 (br s, 1 H), 2.64-2.57 (m, 1 H), 2.31-2.27 (m, 1 H), 2.13-2.03 (m, 3 H), 1.86 (ddd, $J=13.2$, 6.6, $1.8 \mathrm{~Hz}, 1 \mathrm{H}), 1.82-1.68$ (m, 2 H), 1.63-1.52 (m, $1 \mathrm{H}), 1.50-1.43$ (m, $1 \mathrm{H}), 1.04$ (s, $9 \mathrm{H}), 0.93$ (d, $J=$ $7.0 \mathrm{~Hz}, 3 \mathrm{H}), 0.88$ (s, $9 \mathrm{H}), 0.07$ (s, $3 \mathrm{H}), 0.06$ (s, $3 \mathrm{H}) ;{ }^{13} \mathrm{C}$ NMR (150 MHz, $\mathrm{CDCl}_{3}$ ): $\delta=139.9,136.50$, 136.46, 134.4, 134.34, 134.31, 130.47, 130.44, 128.52, 128.48, 115.3, 115.2, 82.9, 82.5, 78.6, 78.4, 76.6, 75.6, 75.4, 74.5, 38.5, 38.3, 38.1, 37.6, 35.0, 34.8, 34.1, 33.7, 31.8, 31.1, 26.8, 19.2, 17.0, 14.9, -4.6, 4.9; HR-MS [Matrix-assisted laser desorption / ionization (MALDI)]: calcd for $\mathrm{C}_{35} \mathrm{H}_{56} \mathrm{O}_{4} \mathrm{Si}_{2} \mathrm{Na}^{+}[\mathrm{M}+$ $\mathrm{Na}^{+}$]: 619.3609, found 619.3602.

Lactone 40. The mixture of secondary alcohols 39 (40.0 mg, $0.067 \mathrm{mmol}, 1.0$ equiv) was dissolved in a 10:2:1 mixture of $t$-butanol:THF:water $(13 \mathrm{~mL})$ at $25{ }^{\circ} \mathrm{C}$. To this was added $N$-methylmorpholine- $N$ -

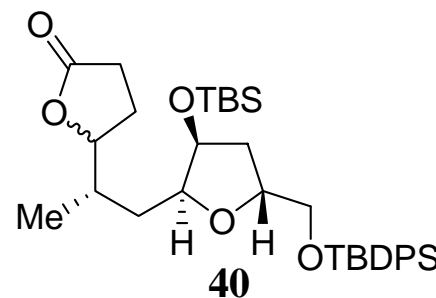

40 oxide (16 mg, $0.134 \mathrm{mmol}, 2.0$ equiv) followed by a $2.5 \%$ solution of osmium tetroxide in $t$-butanol $(0.002 \mathrm{~mL}, 0.002 \mathrm{mmol}, 0.03$ equiv). The reaction mixture was allowed to stir at $25{ }^{\circ} \mathrm{C}$ for $12 \mathrm{~h}$, then a pH 7 buffered saline solution $(20 \mathrm{~mL})$ was added followed by sodium periodate (72 $\mathrm{mg}$, $0.34 \mathrm{mmol}, 5.0$ equiv). The suspension was stirred vigorously for $5 \mathrm{~h}$ and then the reaction mixture was quenched with saturated aqueous $\mathrm{Na}_{2} \mathrm{~S}_{2} \mathrm{O}_{3}(50 \mathrm{~mL})$ and extracted with EtOAc $(3 \times 40 \mathrm{~mL})$. The combined organic layers were dried $\left(\mathrm{MgSO}_{4}\right)$, concentrated, and carried on to the next step without further purification. The resulting lactols were dissolved in $\mathrm{CH}_{2} \mathrm{Cl}_{2}(50 \mathrm{~mL})$ at $25^{\circ} \mathrm{C}$ followed by the addition of $N$-iodosuccinimide (92.7 mg, $0.414 \mathrm{mmol}, 5.0$ equiv), and tetra- $n$-butylammonium iodide (61.2 mg, $0.134 \mathrm{mmol}, 2$ equiv). The resulting dark solution was stirred at $25{ }^{\circ} \mathrm{C}$ for $1 \mathrm{~h}$ and was then 
quenched with saturated aqueous $\mathrm{Na}_{2} \mathrm{~S}_{2} \mathrm{O}_{3}(100 \mathrm{~mL})$. The biphasic mixture was extracted with $\mathrm{CH}_{2} \mathrm{Cl}_{2}$ $(3 \times 80 \mathrm{~mL})$ and the combined organic extracts were dried $\left(\mathrm{MgSO}_{4}\right)$, filtered, concentrated, and purified by flash column chromatography (silica gel, EtOAc:hexanes 1:7) to give a 1:1 ratio of the desired lactone 40 (36.3 mg, $0.061 \mathrm{mmol}$, 91\% over two steps) as a colorless oil. 40 (mixture of epimers ca 1:1): $R_{\mathrm{f}}=0.66$ (silica gel, EtOAc:hexanes 2:3); IR (film) $v_{\max }=2929,2856,1778,1458,1428,1186$, $1113 \mathrm{~cm}^{-1}$; ${ }^{1} \mathrm{H}$ NMR (500 MHz, $\left.\mathrm{CDCl}_{3}\right): \delta=7.72-7.67$ (m, $\left.4 \mathrm{H}\right), 7.42-7.37$ (m, $\left.6 \mathrm{H}\right), 4.34(\mathrm{q}, J=6.5$ Hz, 1 H), 4.29-4.27 (m, 2 H), 4.01-3.99 (m, 1 H), 3.79 (dd, $J=11.0,4.0$ Hz, 1 H), 3.61 (dd, $J=11.0$, 4.0 Hz, 1 H), 2.54-2.48 (m, 2 H), 2.28-2.23 (m, 1 H), 2.20-2.14 (m, 2 H), 2.02-1.95 (m, 2 H), 1.88 (dd, $J=$ 12.5, $6.5 \mathrm{~Hz}, 1 \mathrm{H}$ ), 1.82 (ddd, $J=14.0,10.5,3.5 \mathrm{~Hz}, 1 \mathrm{H}), 1.05$ (s, $9 \mathrm{H}), 1.02$ (d, $J=6.5 \mathrm{~Hz}, 3 \mathrm{H}$ ), 0.90 (s, $9 \mathrm{H}), 0.08$ (s, $3 \mathrm{H}), 0.07$ (s, $3 \mathrm{H}) ;{ }^{13} \mathrm{C}$ NMR (125 MHz, $\mathrm{CDCl}_{3}$ ): $\delta=177.42,177.40,135.62$, 135.59, 135.51, 135.49, 133.6, 133.5, 133.41, 129.59, 129.58, 129.44, 127.60, 127.56, 85.6, 85.3, 80.0, 79.9, 77.5, 77.3, 74.5, 74.1, 66.4, 66.0, 37.4, 35.2, 35.1, 33.3, 32.9, 29.0, 27.2, 26.8, 25.8, 24.2, 19.2, 18.8, 18.1, 14.3, -4.6, -5.0; HR-MS [Matrix-assisted laser desorption / ionization (MALDI)]: calcd for $\mathrm{C}_{34} \mathrm{H}_{52} \mathrm{O}_{5} \mathrm{Si}_{2} \mathrm{Na}^{+}\left[\mathrm{M}+\mathrm{Na}^{+}\right]:$619.3295, found 619.3426.

Sulfoxide Cyclization Precursor 15. A solution of sulfoxide 19 (0.104 g, 0.369 mmol, 1.5 equiv) in THF (1.2 mL) was added to a solution of LDA $(0.245 \mathrm{~mL}, 1.0 \mathrm{M}$ in THF, $0.246 \mathrm{mmol}, 1.0$ equiv) in

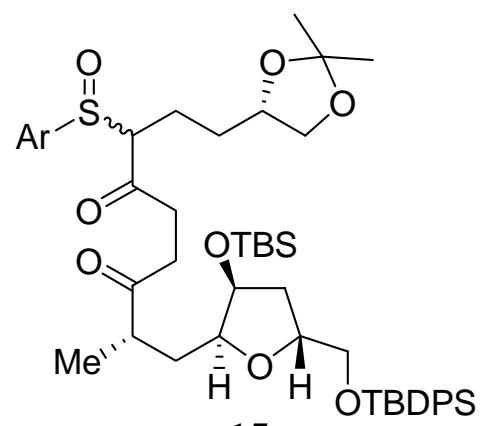

15 THF $(1.5 \mathrm{~mL})$ at $-78^{\circ} \mathrm{C}$. The solution was stirred at $-78{ }^{\circ} \mathrm{C}$ for $25 \mathrm{~min}$ and then a solution of lactone 40 (0.176 g, $0.295 \mathrm{mmol}, 1.0$ equiv) in THF (1.5 $\mathrm{mL}$ ) was added. The reaction mixture was allowed to stir at $-78{ }^{\circ} \mathrm{C}$ for 20 min and then at $0{ }^{\circ} \mathrm{C}$ for $20 \mathrm{~min}$. The reaction mixture was then quenched with saturated aqueous $\mathrm{NH}_{4} \mathrm{Cl}(20 \mathrm{~mL})$ and extracted with EtOAc $(3 \times 20$ $\mathrm{mL})$. The combined organic extracts were dried $\left(\mathrm{MgSO}_{4}\right)$, filtered through a short pad of celite, concentrated and the crude sulfoxide product $\mathbf{4 1}$ was carried on to the next step without further purification. The crude sulfoxide 41 (0.174 g, $0.198 \mathrm{mmol}, 1.0$ equiv) was dissolved in $\mathrm{CH}_{2} \mathrm{Cl}_{2}(3.0 \mathrm{~mL})$ and cooled to $0{ }^{\circ} \mathrm{C}$. To this solution was added $\mathrm{NaHCO}_{3}(200 \mathrm{mg}, 3.69 \mathrm{mmol}, 10.0$ equiv) followed by Dess-Martin periodinane $(0.313 \mathrm{~g}, 0.738 \mathrm{mmol}, 2.0$ equiv). The suspension was 
stirred for $40 \mathrm{~min}$ and then the reaction was quenched with saturated aqueous $\mathrm{Na}_{2} \mathrm{~S}_{2} \mathrm{O}_{3}(10 \mathrm{~mL})$. The biphasic mixture was then extracted with $\mathrm{Et}_{2} \mathrm{O}(3 \times 10 \mathrm{~mL})$ and the combined organic extracts were dried $\left(\mathrm{MgSO}_{4}\right)$, concentrated and purified by flash column chromatography (silica gel, EtOAc:hexanes 2:3) to give a 1:1 ratio of sulfoxide cyclization precursors 15 ( $0.213 \mathrm{~g}, 0.280 \mathrm{mmol}, 76 \%$ over two steps) as a colorless oil. 15 (mixture of epimers ca 1:1): $R_{\mathrm{f}}=0.40$ (silica gel, EtOAc:hexanes 2:3); IR (film) $v_{\max }=2930,2878,1725,1719,1465,1432,1277,1070 \mathrm{~cm}^{-1} ;{ }^{1} \mathrm{H}$ NMR $\left(400 \mathrm{MHz}, \mathrm{CDCl}_{3}\right): \delta=7.71-$ 7.67 (m, 4 H), 7.42-7.35 (m, 6 H), 4.29-4.27 (m, 2 H), 4.08-4.00 (m, 2 H), 3.97-3.94 (m, 1 H), 3.943.92 (m, 4 H), 3.75 (dd, $J=11.0,4.0$ Hz, 1 H), 3.61 (dd, $J=11.0,4.0$ Hz, 1 H), 3.51-3.48 (m, 1 H), 3.02 (dt, $J=12.2,2.2 \mathrm{~Hz}, 1 \mathrm{H}$ ), 2.93 (dt, $J=12.2,2.2 \mathrm{~Hz}, 1 \mathrm{H}), 2.84$ (ddd, $J=16.3$, 11.3, $5.7 \mathrm{~Hz}, 1 \mathrm{H}$ ), 2.74 (ddd, $J=15.8,9.7,5.7$ Hz, 1 H), 2.64-2.60 (m, 2 H), 2.27-2.22 (m, 1 H), 2.15-2.11 (m, 1 H), 2.09-2.04 (m, 2 H), 2.02-1.92 (m, 4 H), 1.89-1.86 (m, 1 H), 1.84-1.77 (m, 1 H), 1.73-1.66 (m, 1 H), 1.37 (s, 3 H), 1.31-1.29 (m, 1 H), 1.28 (s, 3 H), 1.17-1.13 (m, 1 H), 1.04 (s, 9 H), 0.96 (d, J = 7.0 Hz, 3 H), 0.88 (s, 9 H), 0.07 (s, $3 \mathrm{H}), 0.06$ (s, $3 \mathrm{H}) ;{ }^{13} \mathrm{C}$ NMR (150 MHz, $\left.\mathrm{CDCl}_{3}\right): \delta=204.1,203.2,135.6,135.5,135.4$, 133.72, 133.69, 133.6, 129.65, 129.63, 129.5, 127.6, 127.5, 113.1, 113.0, 112.1, 80.3, 80.1, 77.3, 77.2, 75.3, 74.4, 74.2, 69.0, 66.3, 66.2, 65.0, 64.9, 61.2, 37.5, 35.8, 35.6, 34.3, 31.2, 31.1, 30.2, 30.0, 28.7, 28.4, 28.3, 27.6, 26.8, 26.6, 26.5, 25.8, 25.6, 24.7, 23.9, 19.2, 14.1, -4.5, -4.9; HR-MS [Matrix-assisted laser desorption / ionization (MALDI)]: calcd for $\mathrm{C}_{49} \mathrm{H}_{72} \mathrm{O}_{8} \mathrm{SSi}_{2} \mathrm{Na}^{+}\left[\mathrm{M}+\mathrm{Na}^{+}\right]$: 899.4384, found 899.4396.

Tetracycle 42. Sulfoxide cyclization precursor 15 (20.0 mg, $0.024 \mathrm{mmol}, 1.0$ equiv) was dissolved in $\mathrm{CH}_{2} \mathrm{Cl}_{2}(2 \mathrm{~mL})$ and cooled to $-78{ }^{\circ} \mathrm{C}$. To the solution was added TMSOTf $(0.013 \mathrm{~mL}, 0.072 \mathrm{mmol}, 3.0$

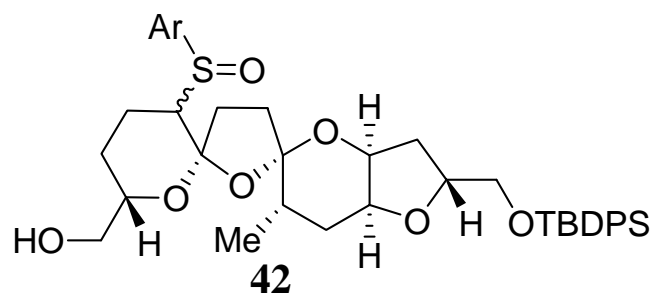
equiv) and the reaction mixture was allowed to warm to $0{ }^{\circ} \mathrm{C}$ over 3 h. The reaction mixture was then quenched with saturated aqueous $\mathrm{NaHCO}_{3}(3 \mathrm{~mL})$ and extracted with $\mathrm{CH}_{2} \mathrm{Cl}_{2}(3 \times 3 \mathrm{~mL})$. The combined organic extracts were dried $\left(\mathrm{Na}_{2} \mathrm{SO}_{4}\right)$, concentrated and purified by flash column chromatography (silica gel, EtOAc:hexanes 1:1) to afford a 1:1 ratio of tetracycles 42 (10.6 mg, $0.015 \mathrm{mmol}, 62 \%)$ as a colorless oil. 42 (mixture of epimers ca 
1:1): $R_{\mathrm{f}}=0.35$ (silica gel, EtOAc:hexanes 1:1); IR (film) $v_{\max }=3420,2920,2889,1725,1462,1280$, $1165 \mathrm{~cm}^{-1}$; ${ }^{1} \mathrm{H}$ NMR (600 MHz, $\left.\mathrm{CDCl}_{3}\right): \delta=7.71-7.67$ (m, $\left.4 \mathrm{H}\right), 7.52-7.48(\mathrm{~m}, 2 \mathrm{H}), 7.42-7.30(\mathrm{~m}, 8$ H), 4.42 (br s, 1 H), 4.39-4.32 (m, 1 H), 4.03-3.96 (m, 2 H), 3.77 (dd, $J=11.0,4.0$ Hz, 1 H), 3.62 (dd, $J$ = 11.0, 4.0 Hz, $1 \mathrm{H}), 3.54-3.51$ (m, $1 \mathrm{H}), 3.50-3.47$ (m, $1 \mathrm{H}), 3.04-2.97$ (m, $1 \mathrm{H})$, 2.91-2.87 (m, $1 \mathrm{H})$, 2.50-2.42 (m, 1 H), 2.30-2.22 (m, 5 H), 2.21-2.18 (m, 1 H), 2.04-1.91 (m, 4 H), 1.80-1.62 (m, 3 H), 1.38-1.26 (m, $1 \mathrm{H}), 1.18$ (d, $J=7.0 \mathrm{~Hz}, 3 \mathrm{H}), 1.14-1.10$ (m, $1 \mathrm{H}), 1.11$ (s, $9 \mathrm{H}) ;{ }^{13} \mathrm{C}$ NMR $(150 \mathrm{MHz}$, $\left.\mathrm{CDCl}_{3}\right): \delta=135.6,135.5,133.5,133.4,129.6,129.5,127.62,127.60,127.5,111.0,110.8,110.1,78.8$, 76.4, 76.1, 74.6, 72.4, 72.0, 66.0, 65.6, 65.2, 56.6, 36.9, 35.8, 35.1, 35.0, 34.4, 31.9, 31.6, 31.0, 30.7, 26.8, 26.4, 25.6, 25.4, 25.2, 23.8, 23.2, 19.2, 19.0, 16.0; HR-MS [Matrix-assisted laser desorption / ionization (MALDI)]: calcd for $\mathrm{C}_{40} \mathrm{H}_{52} \mathrm{O}_{7} \mathrm{SSiNa}^{+}\left[\mathrm{M}+\mathrm{Na}^{+}\right]$: 727.3100, found 727.3110.

ABCD ring system 44. To a solution of tetracycle 42 (11.2 mg, 0.016 mmol, 1.0 equiv) in $\mathrm{CH}_{2} \mathrm{Cl}_{2}(1$ $\mathrm{mL})$ at $25{ }^{\circ} \mathrm{C}$ was added pyridine $(0.013 \mathrm{~mL}, 0.16 \mathrm{mmol}, 10.0$ equiv), followed by benzoyl chloride OTBDS then allowed to stir at $25{ }^{\circ} \mathrm{C}$ for $12 \mathrm{~h}$ and was then quenched 44 with saturated aqueous $\mathrm{NaHCO}_{3}(2 \mathrm{~mL})$. The biphasic mixture was extracted with $\mathrm{CH}_{2} \mathrm{Cl}_{2}(3 \times 2 \mathrm{~mL})$ and the combined organic extracts were dried $\left(\mathrm{Na}_{2} \mathrm{SO}_{4}\right)$, concentrated, and the obtained crude benzoate 43 was used in the next step without any further purification. Crude benzoate 43 was then dissolved in toluene $(2 \mathrm{~mL})$ at $25^{\circ} \mathrm{C}$ in a flask equipped with a reflux condenser. To the solution was added $\mathrm{P}(\mathrm{OMe})_{3}(0.011 \mathrm{~mL}, 0.096 \mathrm{mmol}, 6.0$ equiv) and the resulting reaction mixture was heated to reflux for $12 \mathrm{~h}$. The reaction mixture was then cooled to $25{ }^{\circ} \mathrm{C}$, concentrated and purified by flash column chromatography (silica gel, EtOAc:hexanes 1:1) to afford ABCD ring system 44 (4.7 mg, $0.007 \mathrm{mmol}$, 43\% over two steps) as a colorless oil. 44: $R_{\mathrm{f}}=0.42$ (silica gel, EtOAc:hexanes 1:1); IR (film) $v_{\max }=2927,2888,1724,1462,1378,1272,1113,1070 \mathrm{~cm}^{-1} ;{ }^{1} \mathrm{H}$ NMR (600 MHz, $\left.\mathrm{CDCl}_{3}\right): \delta=7.68-7.65$ (m, 5 H), 7.48-7.35 (m, $\left.10 \mathrm{H}\right)$, 5.98-5.96 (m, $\left.1 \mathrm{H}\right), 5.64$ (d, $J=$ 10.0 Hz, 1 H), 4.42-4.37 (m, 3 H), 4.32-4.27 (m, 2 H), 3.84 (br s, 1 H), 3.64 (dd, J = 11.0, 4.0 Hz, 1 H), 3.60 (dd, $J=11.0,4.0$ Hz, 1 H), 2.28-2.23 (m, 1 H), 2.11 (t, $J=8.0$ Hz, 2 H), 1.92-1.71 (m, 4 H), $1.46-$ 
1.42 (m, $1 \mathrm{H}), 1.30-1.20$ (m, $2 \mathrm{H}), 1.03$ (s, $9 \mathrm{H}), 0.97-0.95$ (m, $1 \mathrm{H}), 0.87$ (d, J = $7.0 \mathrm{~Hz}, 3 \mathrm{H}) ;{ }^{13} \mathrm{C}$ NMR $\left(150 \mathrm{MHz}, \mathrm{CDCl}_{3}\right): \delta=166.6,135.6,133.6,133.0,130.9,130.1,129.7,129.6,128.8,128.7,128.4$, 127.6, 126.5, 109.4, 104.9, 78.5, 76.5, 73.3, 67.5, 67.1, 66.3, 53.4, 37.3, 35.9, 34.9, 31.1, 29.7, 26.8, 26.5, 19.3, 19.2, 15.8; HR-MS [Matrix-assisted laser desorption / ionization (MALDI)]: calcd for $\mathrm{C}_{40} \mathrm{H}_{48} \mathrm{O}_{7} \mathrm{SiNa}^{+}\left[\mathrm{M}+\mathrm{Na}^{+}\right]:$691.3061, found 691.3044 .

Ketone 45. Secondary alcohol 39 (44.15 g, 74.0 mmol, 1.0 equiv) was dissolved in $\mathrm{CH}_{2} \mathrm{Cl}_{2}(1.0 \mathrm{~L})$ and cooled to $0{ }^{\circ} \mathrm{C}$. Dess-Martin periodinane (62.7 g, $148 \mathrm{mmol}, 2.0$ equiv) was added portionwise and then

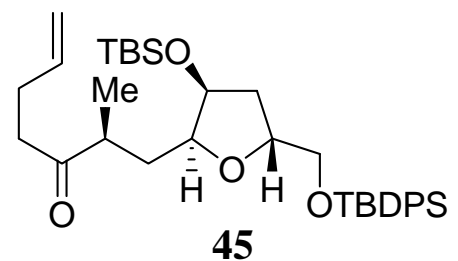
the resulting reaction mixture was allowed to warm to $25{ }^{\circ} \mathrm{C}$ over $4 \mathrm{~h}$. The reaction mixture was then quenched with saturated aqueous $\mathrm{Na}_{2} \mathrm{~S}_{2} \mathrm{O}_{3}$ (125 $\mathrm{mL})$ and saturated aqueous $\mathrm{NaHCO}_{3}(250 \mathrm{~mL})$. The biphasic mixture was then extracted with $\mathrm{CH}_{2} \mathrm{Cl}_{2}(3 \times 300 \mathrm{~mL})$ and the combined organic extracts were dried $\left(\mathrm{MgSO}_{4}\right)$, concentrated, and then purified by flash column chromatography (silica gel, EtOAc:hexanes 1:9) to give ketone 45 (40.9 g, $68.8 \mathrm{mmol}, 95 \%)$ as a colorless oil. 45: $R_{\mathrm{f}}=0.41$ (silica gel, EtOAc: hexanes 1:9); $[\alpha]_{\mathrm{D}}{ }^{25}=+12.1\left(\mathrm{CHCl}_{3}, c=1.70\right)$; IR (film) $v_{\max }=3070,2928,1713,1427$, 1112, 834, $702 \mathrm{~cm}^{-1}$; ${ }^{1} \mathrm{H}$ NMR (600 MHz, $\left.\mathrm{CDCl}_{3}\right): \delta=7.70-7.65$ (m, $\left.4 \mathrm{H}\right), 7.47-7.35$ (m, $\left.6 \mathrm{H}\right), 5.87$ (ddd, $J=17.0,10.5,6.6 \mathrm{~Hz}, 1 \mathrm{H}), 5.02$ (dd, $J=17.0,2.0 \mathrm{~Hz}, 1 \mathrm{H}), 4.99$ (dd, $J=10.5,2.0 \mathrm{~Hz}, 1 \mathrm{H}$ ), 4.29 (br s, 1 H), 4.22 (ddd, $J=14.6,9.2,4.9 \mathrm{~Hz}, 1 \mathrm{H}$ ), 3.90 (dt, $J=9.9,3.6 \mathrm{~Hz}, 1 \mathrm{H}), 3.73$ (dd, $J=11.0,4.0$ Hz, 1 H), 3.59 (dd, $J=11.0,4.0$ Hz, 1 H), 2.73 (m, 1 H), 2.59-2.56 (m, 2 H), 2.31-2.27 (m, 2 H), 2.11 (m, 1 H), 2.01 (ddd, $J=9.7,7.9,4.0 \mathrm{~Hz}, 1 \mathrm{H}), 1.88$ (ddd, $J=12.7,6.5,2.2 \mathrm{~Hz}, 1 \mathrm{H}), 1.39-1.36$ (m, $1 \mathrm{H}$ ), 1.08 (d, $J=7.0$ Hz, 3 H), 1.04 (s, 9 H), 0.88 (s, 9 H), 0.07 (s, 3 H), 0.06 (s, 3 H); ${ }^{13} \mathrm{C}$ NMR (150 MHz, $\left.\mathrm{CDCl}_{3}\right): \delta=213.8,137.5,135.7,135.5,133.6,133.5,129.6,129.5,127.6,127.5,114.9,80.3,77.2,73.9$, 66.0, 43.4, 40.0, 37.3, 32.9, 27.7, 26.7, 25.7, 19.2, 18.0, 16.0, -4.5, -5.0; HR-MS [Matrix-assisted laser desorption / ionization (MALDI)]: calcd for $\mathrm{C}_{35} \mathrm{H}_{54} \mathrm{O}_{4} \mathrm{Si}_{2} \mathrm{Na}^{+}\left[\mathrm{M}+\mathrm{Na}^{+}\right]$: 617.3453, found 617.3458.

Acetal 46. Ketone 45 (40.8 g, $68.6 \mathrm{mmol}, 1.0$ equiv) was dissolved in ethylene glycol (82 mL) and triethyl orthoformate $(82 \mathrm{~mL})$ at $25{ }^{\circ} \mathrm{C}$. To the solution was added p-TsOH $(0.21 \mathrm{~g}, 1.1 \mathrm{mmol}, 0.02$ 
equiv) and the resulting reaction mixture was heated at $55^{\circ} \mathrm{C}$ for $2.5 \mathrm{~h}$. The reaction mixture was cooled to $25^{\circ} \mathrm{C}$ and then quenched with an aqueous $\mathrm{NaOH}$ solution $(1 \mathrm{M}, 110 \mathrm{~mL})$. The biphasic mixture was

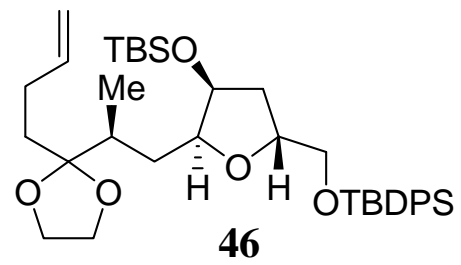
then extracted with EtOAc $(3 \times 300 \mathrm{~mL})$ and the combined organic extracts were dried $\left(\mathrm{MgSO}_{4}\right)$, concentrated, and then purified by flash column chromatography (silica gel, EtOAc:hexanes 1:9) to afford acetal 46 (41.5 g, 64.9 mmol, 98\%) as a colorless oil. 46: $R_{\mathrm{f}}=0.40$ (silica gel, EtOAc: hexanes 1:9); $[\alpha]_{\mathrm{D}}{ }^{25}=+2.7\left(\mathrm{CHCl}_{3}, c=2.30\right)$; IR (film) $v_{\max }=2953,2856,1471,1255,1112,834,702$ $\mathrm{cm}^{-1} ;{ }^{1} \mathrm{H}$ NMR (600 MHz, $\mathrm{CDCl}_{3}$ ): $\delta=7.70$ (dd, $J=19.3,7.0 \mathrm{~Hz}, 4 \mathrm{H}$ ), 7.42-7.35 (m, $6 \mathrm{H}$ ), 5.84 (ddd, $J$ = 17.0, 10.0, $6.1 \mathrm{~Hz}, 1 \mathrm{H}), 5.01$ (d, $J=17.0 \mathrm{~Hz}, 1 \mathrm{H}), 4.92$ (d, $J=10.0 \mathrm{~Hz}, 1 \mathrm{H}), 4.31-4.28$ (m, $1 \mathrm{H})$, 4.27 (br s, 1 H), 3.97 (dt, $J=10.0,3.1 \mathrm{~Hz}, 1 \mathrm{H}$ ), 3.94 (br s, 4 H), 3.76 (dd, $J=11.0,4.0 \mathrm{~Hz}, 1 \mathrm{H}$ ), 3.61 (dd, $J=11.0,4.0 \mathrm{~Hz}, 1 \mathrm{H}), 2.17-2.11$ (m, $4 \mathrm{H}), 1.94$ (ddd, $J=13.6,10.5,3.1 \mathrm{~Hz}, 1 \mathrm{H}), 1.89-1.86$ (m, 1 H), 1.76-1.67 (m, 2 H), 1.16 (ddd, $J=13.6,10.9,3.1$ Hz, 1 H), 1.05 (s, 9 H), 0.96 (d, J = 7.0 Hz, 3 H), 0.90 (s, $9 \mathrm{H}), 0.07$ (s, $3 \mathrm{H}), 0.06$ (s, $3 \mathrm{H}) ;{ }^{13} \mathrm{C}$ NMR (150 MHz, $\mathrm{CDCl}_{3}$ ): $\delta=138.9,135.64,135.60$, 133.7, 133.6, 129.6, 129.5, 127.61, 127.58, 113.9, 113.6, 80.4, 77.2, 74.4, 66.2, 65.24, 65.20, 37.4, 36.2, 33.4, 31.1, 27.6, 26.7, 25.8, 19.2, 18.1, 14.0, -4.5, -4.9; HR-MS [Matrix-assisted laser desorption / ionization (MALDI)]: calcd for $\mathrm{C}_{37} \mathrm{H}_{58} \mathrm{O}_{5} \mathrm{Si}_{2} \mathrm{Na}^{+}\left[\mathrm{M}+\mathrm{Na}^{+}\right]$: 661.3715, found 661.3719.

Aldehyde 47. The acetal 46 (41.5 g, $64.9 \mathrm{mmol}, 1.0$ equiv) was dissolved in a 10:2:1 mixture of $t^{-}$ butanol:THF:water $(600 \mathrm{~mL})$ at $25^{\circ} \mathrm{C}$. To the solution was added $N$-methylmorpholine- $N$-oxide $(15.3 \mathrm{~g}$,

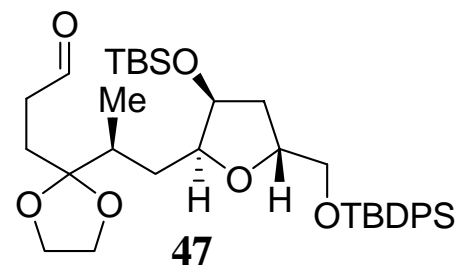

47 $130 \mathrm{mmol}, 2.0$ equiv) followed by a $2.5 \%$ solution of osmium tetroxide in $t$ butanol (3.5 mL, $1.95 \mathrm{mmol}, 0.03$ equiv). The reaction mixture was allowed to stir at $25{ }^{\circ} \mathrm{C}$ for $12 \mathrm{~h}$, at which time a pH 7 buffered saline solution (500 $\mathrm{mL}$ ) was added followed by the portionwise addition of sodium periodate (69.6 g, 325 mmol, 5.0 equiv). The resulting suspension was stirred vigorously for $5 \mathrm{~h}$ and then quenched with saturated aqueous $\mathrm{Na}_{2} \mathrm{~S}_{2} \mathrm{O}_{3}(400 \mathrm{~mL})$. The biphasic mixture was extracted with EtOAc (3 $\times 500 \mathrm{~mL})$ and the combined organic extracts were dried $\left(\mathrm{MgSO}_{4}\right)$, concentrated, and then purified by flash column chromatography (silica gel, EtOAc:hexanes 1:1) to afford aldehyde 47 (41.5 g, 64.9 mmol, 
100\%) as a colorless oil. 47: $R_{\mathrm{f}}=0.65$ (silica gel, ethyl acetate: hexanes 3:7); $[\alpha]_{\mathrm{D}}{ }^{25}=-2.9\left(\mathrm{CHCl}_{3}, c=\right.$ 1.20); IR (film) $v_{\max }=2928,2855,1723,1255,1111,834,702 \mathrm{~cm}^{-1} ;{ }^{1} \mathrm{H}$ NMR (600 MHz, $\left.\mathrm{CDCl}_{3}\right): \delta=$ 9.66 (t, $J=2.0 \mathrm{~Hz}, 1 \mathrm{H}), 7.71-7.67$ (m, 4 H), 7.42-7.34 (m, $6 \mathrm{H}), 4.29$ (m, $1 \mathrm{H}), 4.26$ (br s, $1 \mathrm{H}), 3.95$ (dt, $J=7.0,2.6 \mathrm{~Hz}, 1 \mathrm{H}), 3.93-3.88$ (m, $4 \mathrm{H}), 3.76$ (dd, $J=11.0,3.5 \mathrm{~Hz}, 1 \mathrm{H}), 3.60$ (dd, $J=11.0,3.5$ Hz, 1 H), 2.45-2.38 (m, 2 H), 2.12 (ddd, $J=12.7$, 8.3, 4.8 Hz, 1 H), 2.07-1.99 (m, 3 H), 1.94 (ddd, $J=$ 13.1, 4.7, $2.6 \mathrm{~Hz}, 1 \mathrm{H}$ ), 1.88 (ddd, $J=12.7,7.0,2.1 \mathrm{~Hz}, 1 \mathrm{H}), 1.14$ (ddd, $J=13.6,10.9,3.1 \mathrm{~Hz}, 1 \mathrm{H}$ ), 1.04 (s, $9 \mathrm{H}), 0.96$ (d, $J=7.0 \mathrm{~Hz}, 3 \mathrm{H}), 0.90$ (s, $9 \mathrm{H}), 0.07$ (s, $3 \mathrm{H}), 0.06$ (s, $3 \mathrm{H})$; ${ }^{13} \mathrm{C} \mathrm{NMR}(150 \mathrm{MHz}$, $\left.\mathrm{CDCl}_{3}\right): \delta=202.4,135.6,135.5,133.9,133.8,129.6,129.5,127.6,127.5,113.0,80.2,77.2,74.3,66.2$, 65.1, 65.0, 37.9, 37.4, 36.4, 31.5, 26.7, 26.4, 25.7, 19.2, 14.2, 6.3, -4.5, -4.9; HR-MS [Matrix-assisted laser desorption / ionization (MALDI)]: calcd for $\mathrm{C}_{36} \mathrm{H}_{56} \mathrm{O}_{6} \mathrm{Si}_{2} \mathrm{Na}^{+}\left[\mathrm{M}+\mathrm{Na}^{+}\right]$: 663.3507, found 663.3505.

Cyclization precursor 16. To a solution of dithiane 20 (14.4 g, 58.0 mmol, 3.0 equiv) in THF (140 mL) at $-30{ }^{\circ} \mathrm{C}$ was added $n$-BuLi (36.2 mL, 1.6 M in toluene, $58.0 \mathrm{mmol}$, 3.0 equiv). The resulting solution

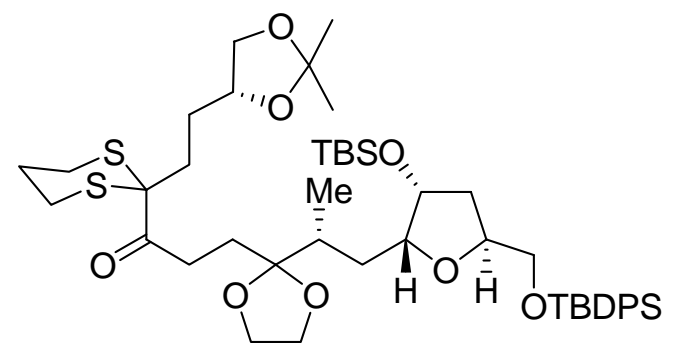

16 was allowed to stir at $-30{ }^{\circ} \mathrm{C}$ for $3 \mathrm{~h}$ at which time a solution of aldehyde 47 (12.39 g, 19.4 mmol, 1.0 equiv) in THF (140 mL) was added dropwise and the reaction mixture was then stirred at $-30{ }^{\circ} \mathrm{C}$ for an additional $1 \mathrm{~h}$ at which time the reaction was quenched with saturated aqueous $\mathrm{NH}_{4} \mathrm{Cl}$ (300 mL). The biphasic mixture was extracted with EtOAc $(3 \times 300 \mathrm{~mL})$ and the combined organic extracts were dried $\left(\mathrm{Na}_{2} \mathrm{SO}_{4}\right)$, concentrated, and used in the next step without any further purification. Crude dithiane 48 (14.9 g, 16.9 mmol, 1.0 equiv) was dissolved in $\mathrm{CH}_{2} \mathrm{Cl}_{2}$ (350 mL) and cooled to $0{ }^{\circ} \mathrm{C}$. To the solution was added pyridine (13.7 mL, $170 \mathrm{mmol}, 10.0$ equiv) and DessMartin periodinane ( $8.55 \mathrm{~g}, 20.3 \mathrm{mmol}, 1.2$ equiv). The reaction mixture was stirred at $0{ }^{\circ} \mathrm{C}$ for $2 \mathrm{~h}$ and then quenched with saturated aqueous $\mathrm{Na}_{2} \mathrm{~S}_{2} \mathrm{O}_{3}(400 \mathrm{~mL})$. The biphasic mixture was then extracted with $\mathrm{CH}_{2} \mathrm{Cl}_{2}(3 \times 300 \mathrm{~mL})$ and the combined organic extracts were dried $\left(\mathrm{Na}_{2} \mathrm{SO}_{4}\right)$, concentrated, and then purified by flash column chromatography (EtOAc:hexanes 1:1) to afford cyclization precursor 16 (13.2 
g, $14.9 \mathrm{mmol}, 88 \%$ ) as a colorless oil. 16: $R_{\mathrm{f}}=0.69$ (silica gel, EtOAc:hexanes 7:3); $[\alpha]_{\mathrm{D}}{ }^{25}=+10.0$ $\left(\mathrm{CHCl}_{3}, c=1.80\right)$; IR (film) $v_{\max }=2928,2856,1701,1458,834,774,703 \mathrm{~cm}^{-1} ;{ }^{1} \mathrm{H} \mathrm{NMR}(600 \mathrm{MHz}$, $\left.\mathrm{CDCl}_{3}\right): \delta=7.71-7.67$ (m, $\left.4 \mathrm{H}\right)$, 7.42-7.35 (m, $\left.6 \mathrm{H}\right), 4.29-4.27$ (m, $\left.2 \mathrm{H}\right), 4.08-4.00$ (m, $\left.2 \mathrm{H}\right), 3.97-3.94$ (m, 1 H), 3.94-3.92 (m, 4 H), 3.75 (dd, $J=11.0,4.0$ Hz, 1 H), 3.61 (dd, $J=11.0,4.0$ Hz, 1 H), 3.513.48 (m, $1 \mathrm{H}$ ), 3.02 (dt, $J=12.2,2.2 \mathrm{~Hz}, 1 \mathrm{H}$ ), 2.93 (dt, $J=12.2,2.2 \mathrm{~Hz}, 1 \mathrm{H}$ ), 2.84 (ddd, $J=16.3,11.3$, 5.7 Hz, 1 H), 2.74 (ddd, $J=15.8,9.7,5.7$ Hz, 1 H), 2.64-2.60 (m, 2 H), 2.27-2.22 (m, 1 H), 2.15-2.11 (m, 1 H), 2.09-2.04 (m, 2 H), 2.02-1.92 (m, 4 H), 1.89-1.86 (m, 1 H), 1.84-1.77 (m, 1 H), $1.73-1.66$ (m, 1 H), 1.37 (s, 3 H), 1.28 (s, 3 H), 1.31-1.26 (m, 1 H), 1.17-1.13 (m, 1 H), 1.04 (s, 9 H), 0.96 (d, J = $7.0 \mathrm{~Hz}, 3 \mathrm{H}), 0.88$ (s, $9 \mathrm{H}), 0.07$ (s, $3 \mathrm{H}), 0.06$ (s, $3 \mathrm{H}) ;{ }^{13} \mathrm{C}$ NMR $\left(150 \mathrm{MHz}, \mathrm{CDCl}_{3}\right): \delta=204.1$, 135.6, 135.5, 133.7, 133.6, 129.6, 129.5, 127.6, 127.5, 113.1, 112.1, 80.3, 77.3, 75.3, 74.4, 69.0, 66.3, 66.2, 65.0, 61.2, 37.5, 35.8, 34.3, 31.2, 31.1, 30.2, 28.7, 28.4, 28.4, 27.6, 26.8, 26.7, 25.8, 25.6, 24.7, 19.2, 14.1, -4.5, -4.9; HR-MS [Matrix-assisted laser desorption / ionization (MALDI)]: calcd for $\mathrm{C}_{47} \mathrm{H}_{74} \mathrm{O}_{8} \mathrm{~S}_{2} \mathrm{Si}_{2} \mathrm{Na}^{+}\left[\mathrm{M}+\mathrm{Na}^{+}\right]:$909.4256, found 909.4257.

Cyclized product 49. Cyclization precursor 16 (5.7 g, $6.4 \mathrm{mmol}, 1.0$ equiv) was dissolved in $\mathrm{CH}_{2} \mathrm{Cl}_{2}$ (300 mL) and cooled to $-78{ }^{\circ} \mathrm{C}$. To the solution was added TMSOTf (1.1 mL, $0.072 \mathrm{mmol}, 3.0$ equiv)

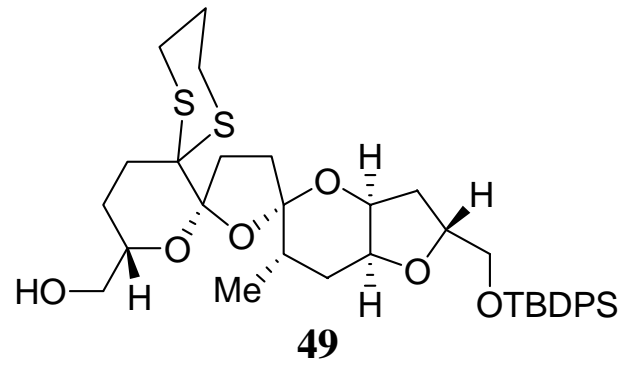
and the reaction mixture was allowed to warm to $-30{ }^{\circ} \mathrm{C}$ over $3 \mathrm{~h}$. The reaction mixture was then quenched with saturated aqueous $\mathrm{NaHCO}_{3}(150 \mathrm{~mL})$. The biphasic mixture was extracted with $\mathrm{CH}_{2} \mathrm{Cl}_{2}(3 \times 200 \mathrm{~mL})$ and the combined organic extracts were dried, concentrated, and then purified by flash column chromatography (silica gel, EtOAc:hexanes 1:1) to afford cyclized product 49 (3.65 g, 5.44 mmol, 85\%) as a colorless oil. 49: $R_{\mathrm{f}}=0.29$ (silica gel, EtOAc:hexanes 3:7); $[\alpha]_{\mathrm{D}}{ }^{25}=-9.9\left(\mathrm{CHCl}_{3}, c=0.70\right)$; IR (film) $v_{\max }=3588,2929,2857,1458,1427,1111,1031 \mathrm{~cm}^{-1} ;{ }^{1} \mathrm{H}$ NMR $\left(600 \mathrm{MHz}, \mathrm{CDCl}_{3}\right): \delta=7.69-$ 7.65 (m, 4 H), 7.42-7.35 (m, 6 H), 4.40 (br s, 1 H), 4.31 (ddd, $J=10.1,6.2,4.0$ Hz, 1 H), 4.16-4.10 (m, $1 \mathrm{H}), 3.98$ (br s, $1 \mathrm{H}), 3.76$ (dd, $J=11.0,4.0 \mathrm{~Hz}, 1 \mathrm{H}), 3.64$ (dd, $J=11.0,4.0 \mathrm{~Hz}, 1 \mathrm{H})$, 3.59-3.56 (m, 2 H), 2.99 (ddd, $J=14.9,12.7,3.1 \mathrm{~Hz}, 1 \mathrm{H}$ ), 2.85 (ddd, $J=14.4,11.8,2.6 \mathrm{~Hz}, 1 \mathrm{H}), 2.67-2.60$ (m, $4 \mathrm{H}$ ), 
2.39 (dt, $J=13.0,4.0 \mathrm{~Hz}, 1 \mathrm{H}), 2.24-2.19$ (m, 1 H), 2.19-2.12 (m, $3 \mathrm{H}), 2.07-2.02$ (m, $3 \mathrm{H}), 1.99-1.93$ (m, 2 H), 1.88-1.71 (m, 3 H), 1.41 (dd, $J=$ 13.1, 2.6 Hz, 1 H), 1.04 (s, 9 H), 1.03 (d, $J=7.0$ Hz, 3 H); ${ }^{13} \mathrm{C}$ NMR $\left(150 \mathrm{MHz}, \mathrm{CDCl}_{3}\right): \delta=135.6,135.5,133.5,133.4,129.6,129.5,127.6,127.5,111.0,110.1$, 78.8, 76.4, 74.6, 72.4, 66.0, 65.6, 56.6, 35.8, 35.1, 34.4, 31.9, 31.0, 30.7, 26.8, 25.6, 25.4, 25.2, 23.8, 19.2, 16.0; HR-MS [Matrix-assisted laser desorption / ionization (MALDI)]: calcd for $\mathrm{C}_{36} \mathrm{H}_{50} \mathrm{O}_{6} \mathrm{~S}_{2} \mathrm{SiNa}^{+}$ $\left[\mathrm{M}+\mathrm{Na}^{+}\right]:$693.2710, found 693.2714.

Pivaloate 50. Cyclized product 49 (3.2 g, $4.8 \mathrm{mmol}$, 1.0 equiv) was dissolved in $\mathrm{CH}_{2} \mathrm{Cl}_{2}(70 \mathrm{~mL}$ ) and cooled to $0{ }^{\circ} \mathrm{C}$. To the solution was added pyridine (3.82 mL, $48 \mathrm{mmol}, 10.0$ equiv), 4-DMAP (116 mg,

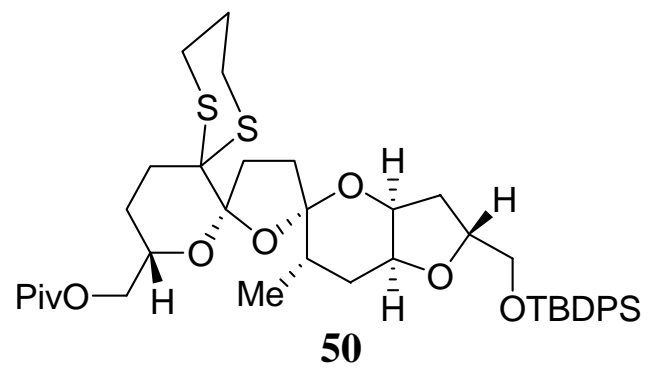
$0.48 \mathrm{mmol}$, 0.1 equiv), and PivCl (1.73 mL, $14.4 \mathrm{mmol}, 3.0$ equiv). The reaction mixture was allowed to warm to $25^{\circ} \mathrm{C}$ over $3 \mathrm{~h}$, then concentrated, and purified by flash column chromatography (silica gel, EtOAc:hexanes 2:3) to afford pivaloate 50 (3.47 g, $4.6 \mathrm{mmol}, 95 \%)$ as a colorless oil. 50: $R_{\mathrm{f}}=$ 0.66 (silica gel, EtOAc:hexanes 4:6); $[\alpha]_{D}^{25}=-11.5\left(\mathrm{CHCl}_{3}, c=0.80\right)$; IR (film) $v_{\max }=2928,2857$, 1731, 1557, 1167, 1111, $1030 \mathrm{~cm}^{-1}$; ${ }^{1} \mathrm{H}$ NMR (600 MHz, $\mathrm{CDCl}_{3}$ ): $\delta=7.70-7.65$ (m, $4 \mathrm{H}$ ), 7.43-7.34 (m, 6 H), 4.38-4.34 (m, 1 H), 4.34-4.30 (m, 1 H), 4.20-4.17 (m, 1 H), 4.12 (dd, $J=11.0,6.0$ Hz, 1 H), 3.98 (dd, $J$ = 11.0, 4.0 Hz, 1 H), 3.97 (br s, 1 H), 3.75 (dd, $J=11.0,4.0$ Hz, 1 H), 3.66 (dd, $J$ = 11.0, 4.0 Hz, 1 H), 2.97 (ddd, $J$ = 14.6, 12.0, 2.6 Hz, 1 H), 2.84 (ddd, $J$ = 14.6, 11.8, 3.2 Hz, 1 H), 2.63 (m, 4 H), 2.42-2.38 (m, 1 H), 2.13-2.00 (m, 9 H), 1.84 (dd, $J$ = 9.4, 2.6 Hz, 2 H), 1.81-1.74 (m, 1 H), 1.24 (s, 9 H), 1.22 (d, $J=7.0 \mathrm{~Hz}, 3 \mathrm{H}), 1.06$ (s, $9 \mathrm{H}) ;{ }^{13} \mathrm{C}$ NMR (150 MHz, $\left.\mathrm{CDCl}_{3}\right): \delta=178.4,135.6,135.5$, 133.6, 133.5, 129.6, 129.5, 127.1, 127.0, 110.7, 109.9, 78.7, 77.6, 76.6, 74.3, 68.9, 66.6, 56.6, 38.8, 36.1, 34.9, 34.2, 32.0, 30.9, 30.4, 27.2, 27.0, 26.8, 25.6, 25.5, 25.1, 24.6, 19.2, 16.2; HR-MS [Matrix-assisted laser desorption / ionization (MALDI)]: calcd for $\mathrm{C}_{41} \mathrm{H}_{58} \mathrm{O}_{7} \mathrm{~S}_{2} \mathrm{SiNa}^{+}\left[\mathrm{M}+\mathrm{Na}^{+}\right]$: 777.3285, found 777.3280. 
Ketone 51. To a solution of pivaloate 50 ( $3.41 \mathrm{~g}, 4.52 \mathrm{mmol}$, 1.0 equiv) in $\mathrm{CH}_{3} \mathrm{CN}\left(40 \mathrm{~mL}\right.$ ) and $\mathrm{H}_{2} \mathrm{O}$

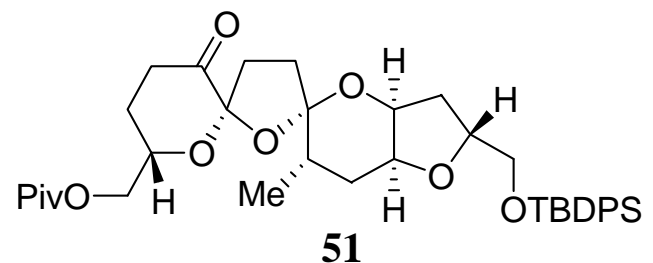
$(10 \mathrm{~mL})$ at $25{ }^{\circ} \mathrm{C}$ was added 2,6-lutidine $(8.4 \mathrm{~mL}, 72.3 \mathrm{mmol}$, 16.0 equiv) followed by $N$-bromosuccinimide $(6.41 \mathrm{~g}, 38.8$ mmol, 8.0 equiv). The reaction mixture was allowed to stir for 2 $\mathrm{h}$ at $25{ }^{\circ} \mathrm{C}$ and was then quenched with saturated aqueous $\mathrm{NaHCO}_{3}(50 \mathrm{~mL})$. The resulting biphasic mixture was extracted with EtOAc $(3 \times 50 \mathrm{~mL})$ and the combined organic extracts were dried $\left(\mathrm{Na}_{2} \mathrm{SO}_{4}\right)$, concentrated, and then purified by flash column chromatography (silica gel, EtOAc:hexanes 2:3) to afford ketone 51 (2.73 g, $4.11 \mathrm{mmol}, 91 \%$ ) as a colorless oil. 51: $R_{\mathrm{f}}=0.64$ (silica gel, EtOAc:hexanes 4:6); $[\alpha]_{\mathrm{D}}{ }^{25}=+1.0\left(\mathrm{CHCl}_{3}\right.$, $c=1.70)$; IR (film) $v_{\max }=2959,2857,1733,1472,1428,1156,1112,1033 \mathrm{~cm}^{-1} ;{ }^{1} \mathrm{H}$ NMR $(600 \mathrm{MHz}$, $\mathrm{CDCl}_{3}$ ): $\delta=7.71-7.64$ (m, 4 H), 7.42-7.35 (m, $6 \mathrm{H}$ ), 4.54 (dddd, $J$ = 13.2, 9.1, 3.8, $2.6 \mathrm{~Hz}, 1 \mathrm{H}$ ), 4.36 (br s, 1 H), 4.33 (br s, 1 H), 4.16 (dd, $J=11.0,6.5$ Hz, 1 H), 4.07 (dd, $J=11.0,4.0$ Hz, 1 H), 3.97 (br s, 1 H), 3.78 (dd, $J=11.0,4.0 \mathrm{~Hz}, 1$ H), 3.66 (dd, $J=11.0,4.0 \mathrm{~Hz}, 1 \mathrm{H}$ ), 2.86 (ddd, $J=14.7,11.2,6.4 \mathrm{~Hz}$, $1 \mathrm{H}$ ), 2.56 (ddd, $J=11.2,7.7,3.0 \mathrm{~Hz}, 1 \mathrm{H}$ ), 2.49 (ddd, $J=14.7,4.4,2.4 \mathrm{~Hz}, 1 \mathrm{H}$ ), 2.18-1.87 (m, 9 H), 1.71 (ddd, $J=16.4,14.8,3.2 \mathrm{~Hz}, 1 \mathrm{H}$ ), 1.23 (s, $9 \mathrm{H}$ ), 1.05 (s, $9 \mathrm{H}$ ), 0.74 (d, $J=7.0 \mathrm{~Hz}, 3 \mathrm{H}$ ); ${ }^{13} \mathrm{C}$ NMR $\left(150 \mathrm{MHz}, \mathrm{CDCl}_{3}\right): \delta=201.6,178.3,135.65,135.61,133.6,133.6,129.59,129.56,127.60,127.58$, 111.3, 107.8, 77.6, 75.5, 73.7, 69.7, 66.2, 66.0, 36.2, 36.1, 34.7, 33.5, 30.7, 29.6, 29.2, 27.2, 26.9, 24.5, 19.3, 17.0; HR-MS [Matrix-assisted laser desorption / ionization (MALDI)]: calcd for $\mathrm{C}_{38} \mathrm{H}_{52} \mathrm{O}_{8} \mathrm{SiNa}^{+}$ $\left[\mathrm{M}+\mathrm{Na}^{+}\right]: 687.3323$, found 687.3327.

Alcohol 52. To a solution of ketone 51 (400 mg, $0.60 \mathrm{mmol}, 1.0$ equiv) in $\mathrm{MeOH}\left(35 \mathrm{~mL}\right.$ ) at $-5{ }^{\circ} \mathrm{C}$ was added $\mathrm{NaBH}_{4}$ (23 mg, $0.60 \mathrm{mmol}, 1.0$ equiv). The reaction mixture was allowed to stir at $-5^{\circ} \mathrm{C}$ for 5

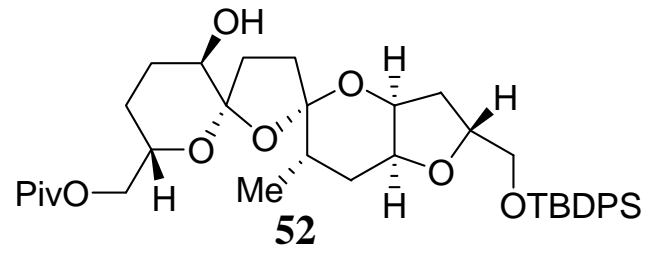
min and was then quenched with saturated aqueous $\mathrm{NH}_{4} \mathrm{Cl}(30$ $\mathrm{mL})$. The biphasic mixture was extracted with $\mathrm{CH}_{2} \mathrm{Cl}_{2}(3 \times 50$ $\mathrm{mL}$ ) and the combined organic layers were dried $\left(\mathrm{Na}_{2} \mathrm{SO}_{4}\right)$, concentrated, and then purified by flash column chromatography (silica gel, EtOAc:hexanes 1:1) to afford a single isomer of alcohol 52 (370 mg, $0.55 \mathrm{mmol}, 92 \%$ ) as a 
colorless oil. 52: $R_{\mathrm{f}}=0.20$ (silica gel, EtOAc:hexanes 1:3); IR (film) $v_{\max }=3648,2930,1730,1457$, 1149, $1109 \mathrm{~cm}^{-1} ;{ }^{1} \mathrm{H}$ NMR (600 MHz, $\mathrm{CDCl}_{3}$ ): $\delta=7.72-7.64$ (m, $\left.4 \mathrm{H}\right), 7.42-7.37$ (m, $\left.6 \mathrm{H}\right), 4.37-4.31$ (m, 1 H), 4.32 (br s, 1 H), 4.14-4.02 (m, 2 H), 4.00-3.92 (m, 2 H), 3.77 (dd, J = 11.0, 4.0 Hz, 1 H), 3.67 (dd, $J=11.0,4.0$ Hz, 1 H), 3.51-3.47 (m, 1 H), 2.25-2.22 (m, 1 H), 2.19-2.03 (m, 5 H), 1.99-1.92 (m, 3 H), 1.82-1.77 (m, 1 H), 1.72-1.65 (m, 2 H), 1.45-1.41 (m, 1 H), 1.22 (s, 9 H), 1.05 (s, 9 H), 0.94 (d, J = 7.0 Hz, $3 \mathrm{H}) ;{ }^{13} \mathrm{C}$ NMR (150 MHz, $\left.\mathrm{CDCl}_{3}\right): \delta=175.3,135.6,135.5,133.5,133.4,129.6,129.5,127.7$, 127.6, 78.6, 76.4, 73.5, 71.4, 69.2, 66.5, 66.0, 38.7, 36.0, 34.6, 34.2, 31.3, 30.7, 29.0, 27.4, 27.2, 26.8, 19.2, 16.6; HR-MS [Matrix-assisted laser desorption / ionization (MALDI)]: calcd for $\mathrm{C}_{38} \mathrm{H}_{54} \mathrm{O}_{8} \mathrm{SiNa}^{+}$ $\left[\mathrm{M}+\mathrm{Na}^{+}\right]:$689.3480, found 689.3481.

Epimerized product 53. To a solution of alcohol 52 (543 mg, $0.82 \mathrm{mmol}, 1.0$ equiv) in $\mathrm{CH}_{2} \mathrm{Cl}_{2}(10 \mathrm{~mL})$ at $0{ }^{\circ} \mathrm{C}$ was added trifluoroacetic acid $(0.20 \mathrm{~mL}, 2.46 \mathrm{mmol}, 3.0$ equiv). The reaction mixture was $\lambda_{\mathrm{H}}$ allowed to stir at $0{ }^{\circ} \mathrm{C}$ for $3 \mathrm{~h}$ and then quenched with $\mathrm{Et}_{3} \mathrm{~N}(0.5$ $\mathrm{mL})$. The resulting solution was concentrated and purified by flash column chromatography (silica gel, EtOAc:hexanes 1:1) to afford epimerized product 53 (300 mg, $0.46 \mathrm{mmol}, 56 \%$ ) as a colorless oil in addition to recovered alcohol 52 (220 mg, $0.33 \mathrm{mmol}, 40 \%$ ) which was resubjected to the reaction conditions. 53: $R_{\mathrm{f}}=0.35$ (silica gel, EtOAc:hexanes 1:3); $[\alpha]_{\mathrm{D}}{ }^{25}=+19.2\left(\mathrm{CHCl}_{3}, c=1.10\right)$; IR (film) $v_{\max }=3640,2928,1730$, 1457, 1111, $1073 \mathrm{~cm}^{-1}$; ${ }^{1} \mathrm{H}$ NMR (600 MHz, $\mathrm{CDCl}_{3}$ ): $\delta=7.70-7.64$ (m, $4 \mathrm{H}$ ), 7.41-7.35 (m, $6 \mathrm{H}$ ), 4.41 (ddd, $J=12.7,7.2,3.9$ Hz, 1 H), 4.11 (br s, 1 H), 4.07-4.03 (m, 1 H), 3.99-3.97 (m, 2 H), 3.88 (br s, 1 H), 3.77 (dd, $J=11.0,4.0$ Hz, 1 H), 3.64 (dd, $J=11.0,4.0$ Hz, 1 H), 3.46-3.40 (m, 1 H), 2.50-2.44 (m, $1 \mathrm{H}), 2.24$ (ddd, $J=18.0,11.0,6.6 \mathrm{~Hz}, 1 \mathrm{H}), 2.16$ (dt, $J=9.2,4.4 \mathrm{~Hz}, 1 \mathrm{H}), 2.13-2.09$ (m, $1 \mathrm{H}$ ), 2.04-1.85 (m, 4 H), 1.83-1.78 (m, 1 H), 1.75-1.65 (m, 3 H), 1.45-1.41 (m, 1 H), 1.18 (s, 9 H), 1.04 (s, $9 \mathrm{H}), 0.95$ (d, $J=7.0 \mathrm{~Hz}, 3 \mathrm{H}) ;{ }^{13} \mathrm{C}$ NMR (150 MHz, $\left.\mathrm{CDCl}_{3}\right): \delta=178.3,135.6,135.5,133.5,133.4$, 129.6, 129.5, 127.6, 127.5, 111.6, 108.6, 78.6, 76.5, 75.8, 68.7, 68.3, 66.7, 65.9, 38.7, 36.0, 33.6, 31.2, 30.2, 28.8, 27.1, 26.9, 26.8, 22.8, 19.2, 15.8; HR-MS [Matrix-assisted laser desorption / ionization (MALDI)]: calcd for $\mathrm{C}_{38} \mathrm{H}_{54} \mathrm{O}_{8} \mathrm{SiNa}^{+}\left[\mathrm{M}+\mathrm{Na}^{+}\right]$: 689.3480, found 689.3475. 
Ketone 54. To a solution of oxallyl chloride ( $0.22 \mathrm{~mL}, 2.5 \mathrm{mmol}, 5.0$ equiv) in $\mathrm{CH}_{2} \mathrm{Cl}_{2}(12 \mathrm{~mL})$ at -78 ${ }^{\circ} \mathrm{C}$ was added DMSO (0.34 mmol, $5.5 \mathrm{mmol}, 11.0$ equiv). The solution was allowed to stir for $20 \mathrm{~min}$ at $\lambda_{\mathrm{H}}$ $-78{ }^{\circ} \mathrm{C}$ and then a solution of epimerized product 55 (0.34 g, $0.51 \mathrm{mmol}, 1.0$ equiv) in $\mathrm{CH}_{2} \mathrm{Cl}_{2}(10 \mathrm{~mL})$ was added and the resulting mixture was allowed to stir at $-78{ }^{\circ} \mathrm{C}$ for $1 \mathrm{~h} . \mathrm{Et}_{3} \mathrm{~N}$ (1.57 mL, $11.0 \mathrm{mmol}, 22.0$ equiv) was then added and the reaction mixture was allowed to warm to $0{ }^{\circ} \mathrm{C}$ over $1 \mathrm{~h}$. The reaction mixture was then quenched with saturated aqueous $\mathrm{NaCl}(30 \mathrm{~mL})$ and extracted with $\mathrm{CH}_{2} \mathrm{Cl}_{2}(3 \times 20 \mathrm{~mL})$. The combined organic extracts were dried $\left(\mathrm{Na}_{2} \mathrm{SO}_{4}\right)$, concentrated, and then purified by flash column chromatography (silica gel, EtOAc:hexanes 1:1) to give ketone 54 (273 mg, $0.41 \mathrm{mmol}, 80 \%$ ) as a colorless oil. 54: $R_{\mathrm{f}}=0.50$ (silica gel, EtOAc:hexanes $1: 3$ ); $[\alpha]_{\mathrm{D}}{ }^{25}=-18.6$ $\left(\mathrm{CHCl}_{3}, c=2.00\right)$; IR (film) $v_{\max }=2958,2857,1731,1645,1427,1282,1111 \mathrm{~cm}^{-1} ;{ }^{1} \mathrm{H}$ NMR $(600$ $\left.\mathrm{MHz}, \mathrm{CDCl}_{3}\right): \delta=7.68-7.64(\mathrm{~m}, 4 \mathrm{H}), 7.41-7.35$ (m, $\left.6 \mathrm{H}\right), 4.56-4.54$ (m, $\left.1 \mathrm{H}\right), 4.43-4.39$ (m, $\left.1 \mathrm{H}\right)$, 4.13 (br s, 1 H), 4.08-4.04 (m, 2 H), 3.86 (br s, 1 H), 3.75 (dd, $J=11.0,4.0$ Hz, 1 H), 3.61 (dd, $J=11.0$, 4.0 Hz, 1 H), 3.01-2.95 (m, 1 H), 2.78-2.73 (m, 1 H), 2.50 (ddd, $J=18.7,4.4,2.6$ Hz, 1 H), 2.28-2.21 (m, 1 H), 2.14-2.05 (m, 3 H), 2.03-1.85 (m, 4 H), 1.71 (dd, $J=12.7,7.4$ Hz, 1 H), 1.47-1.41 (m, 1 H), 1.19 (s, $9 \mathrm{H}), 1.03$ (s, $9 \mathrm{H}), 0.97$ (d, $J=7.0 \mathrm{~Hz}, 3 \mathrm{H}) ;{ }^{13} \mathrm{C}$ NMR (150 MHz, $\left.\mathrm{CDCl}_{3}\right): \delta=201.4,178.3$, 135.6, 135.5, 133.5, 133.4, 129.6, 129.5, 127.6, 127.5, 113.0, 106.7, 78.5, 76.6, 75.6, 68.1, 66.0, 65.9, 38.7, 35.7, 35.6, 33.8, 30.8, 30.0, 29.0, 27.1, 26.8, 22.6, 19.2, 15.9; HR-MS [Matrix-assisted laser desorption / ionization (MALDI)]: calcd for $\mathrm{C}_{38} \mathrm{H}_{52} \mathrm{O}_{8} \mathrm{SiNa}^{+}\left[\mathrm{M}+\mathrm{Na}^{+}\right]$: 687.3323, found 687.3324.

Triflate 56. To a solution of ketone 54 (150 mg, $0.23 \mathrm{mmol}, 1.0$ equiv) and Comin's reagent 55 (472

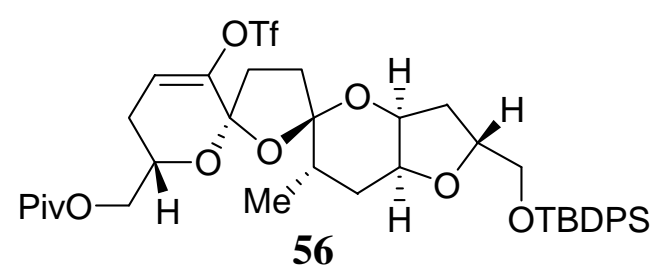

56 mg, $1.15 \mathrm{mmol}, 5.0$ equiv) in THF $(10 \mathrm{~mL})$ at $-78{ }^{\circ} \mathrm{C}$ was added KHMDS (2.17 mL, 0.5 M in toluene, $1.04 \mathrm{mmol}, 4.5$ equiv). The reaction mixture was allowed to stir for $1 \mathrm{~h}$ at $-78{ }^{\circ} \mathrm{C}$ and then quenched with saturated aqueous $\mathrm{NaHCO}_{3}(20 \mathrm{~mL})$. The biphasic mixture was extracted with EtOAc $(3 \times 15 \mathrm{~mL})$ and the combined organic extracts were dried 
( $\mathrm{Na}_{2} \mathrm{SO}_{4}$ ), concentrated, and then purified by flash column chromatography (silica gel, EtOAc:hexanes 1:1) to afford triflate $56(150 \mathrm{mg}, 0.19 \mathrm{mmol}, 83 \%)$ as a colorless oil. 56: $R_{\mathrm{f}}=0.67$ (silica gel, EtOAc:hexanes 1:3); $[\alpha]_{\mathrm{D}}^{25}=-13.3\left(\mathrm{CHCl}_{3}, c=2.40\right)$; IR (film) $v_{\max }=2930,1734,1426,1207,1146$, $1073 \mathrm{~cm}^{-1}$; ${ }^{1} \mathrm{H}$ NMR (600 MHz, $\mathrm{CDCl}_{3}$ ): $\delta=7.68-7.64$ (m, $4 \mathrm{H}$ ), 7.42-7.35 (m, $\left.6 \mathrm{H}\right), 6.03$ (dd, $J=5.7$, $1.3 \mathrm{~Hz}, 1 \mathrm{H}$ ), 4.46-4.43 (m, 1 H), 4.31-4.26 (m, 1 H), 4.16 (br s, 1 H), 4.12-4.06 (m, 2 H), 3.89 (br s, 1 H), 3.79 (dd, $J=11.0,4.0 \mathrm{~Hz}, 1 \mathrm{H}$ ), 3.64 (dd, $J=11.0,4.0 \mathrm{~Hz}, 1 \mathrm{H}$ ), 2.52-2.47 (m, $1 \mathrm{H}$ ), 2.34-2.31 (m, 1 H), 2.28-2.22 (m, 1 H), 2.21 (ddd, $J=17.5,6.1,3.5$ Hz, 1 H), 2.15-2.10 (m, 3 H), 2.04-1.97 (m, 2 H), 1.91-1.88 (m, 1 H), 1.43-1.39 (m, 1 H), 1.19 (s, 9 H), 1.03 (s, 9 H), 0.95 (d, J = 7.0 Hz, 3 H); ${ }^{13} \mathrm{C}$ NMR (150 MHz, $\left.\mathrm{CDCl}_{3}\right): \delta=178.3,145.1,135.6,135.58,133.63,133.60,129.6,129.5,127.63$, 127.60, 127.58, 117.5, 78.8, 76.2, 75.8, 66.1, 66.0, 65.5, 38.7, 35.5, 33.8, 31.9, 30.7, 27.1, 27.0, 26.8, 23.0, 19.2, 15.8; HR-MS [Matrix-assisted laser desorption / ionization (MALDI)]: calcd for $\mathrm{C}_{39} \mathrm{H}_{51} \mathrm{~F}_{3} \mathrm{O}_{10} \mathrm{SSiNa}^{+}\left[\mathrm{M}+\mathrm{Na}^{+}\right]$: 819.2816, found 819.2811.

Alkene 57. To a solution of triflate 56 (150 mg, $0.19 \mathrm{mmol}, 1.0$ equiv) in THF (5 mL) at $25{ }^{\circ} \mathrm{C}$ was added $\mathrm{Pd}\left(\mathrm{PPh}_{3}\right)_{4}$ (46 mg, $0.04 \mathrm{mmol}, 0.2$ equiv) followed by $n-\mathrm{Bu}_{3} \mathrm{SnH}(0.50 \mathrm{~mL}, 1.9 \mathrm{mmol}, 10.0$

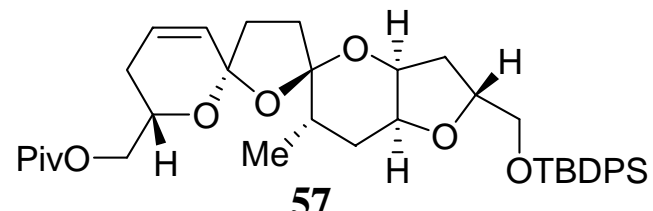

57 equiv). The reaction mixture was allowed to stir for $45 \mathrm{~min}$ at 25 ${ }^{\circ} \mathrm{C}$ and then quenched with saturated aqueous $\mathrm{NaHCO}_{3}(5 \mathrm{~mL})$. The resulting biphasic mixture was extracted with EtOAc $(3 \times 5$ $\mathrm{mL})$ and the combined organic extracts were dried $\left(\mathrm{Na}_{2} \mathrm{SO}_{4}\right)$, concentrated, and then purified by flash column chromatography (silica gel, EtOAc:hexanes 1:1) to afford alkene 57 (109 mg, $0.17 \mathrm{mmol}, 90 \%$ ) as a colorless oil. 57: $R_{\mathrm{f}}=0.51$ (silica gel, EtOAc:hexanes 2:8); $[\alpha]_{\mathrm{D}}{ }^{25}=-14.9\left(\mathrm{CHCl}_{3}, c=1.50\right)$; IR (film) $v_{\max }=3070,2957,2856,1731,1653,1458,1427,1284,1111 \mathrm{~cm}^{-1} ;{ }^{1} \mathrm{H}$ NMR $\left(600 \mathrm{MHz}, \mathrm{CDCl}_{3}\right)$ : $\delta=7.69-7.64$ (m, 4 H), 7.41-7.34 (m, 6 H), 5.96 (ddd, $J=10.0$, 5.6, 2.4 Hz, 1 H), 5.72 (dd, $J=10.0$, $1.2 \mathrm{~Hz}, 1 \mathrm{H}), 4.50-4.44$ (m, 1 H), 4.26-4.20 (m, 1 H), 4.20-4.15 (m, 1 H), 4.08 (d, $J=2.0 \mathrm{~Hz}, 1 \mathrm{H}$ ), 4.06 (d, $J=3.2 \mathrm{~Hz}, 1 \mathrm{H}$ ), 3.89 (d, $J=2.0 \mathrm{~Hz}, 1 \mathrm{H}$ ), 3.76 (dd, $J=11.0,4.0 \mathrm{~Hz}, 1 \mathrm{H}$ ), 3.63 (dd, $J=11.0$, 4.0 Hz, 1 H), 2.26-1.91 (m, 11 H), 1.19 (s, 9 H), 1.03 (s, 9 H), 0.96 (d, $J=7.0$ Hz, 3 H); ${ }^{13} \mathrm{C}$ NMR (150 $\left.\mathrm{MHz}, \mathrm{CDCl}_{3}\right): \delta=178.4,135.6,135.5,133.5,133.4,129.6,129.5,127.63,127.60,127.3,109.6,111.5$, 
103.9, 78.5, 76.5, 75.9, 73.5, 66.5, 66.4, 38.7, 36.1, 35.7, 33.9, 30.8, 27.1, 26.8, 19.2, 17.5, 15.9, 13.5; HR-MS [Matrix-assisted laser desorption / ionization (MALDI)]: calcd for $\mathrm{C}_{38} \mathrm{H}_{52} \mathrm{O}_{7} \mathrm{SiNa}^{+}\left[\mathrm{M}+\mathrm{Na}^{+}\right]$: 671.3374, found 671.3366 .

Alcohol 58. To a solution of alkene 57 (338 mg, $0.60 \mathrm{mmol}, 1.0$ equiv) in $\mathrm{CH}_{2} \mathrm{Cl}_{2}\left(25 \mathrm{~mL}\right.$ ) at $-78{ }^{\circ} \mathrm{C}$ was added DIBAL-H (1.5 mL, $1.0 \mathrm{M}$ in $\mathrm{CH}_{2} \mathrm{Cl}_{2}, 1.5 \mathrm{mmol}, 2.5$ equiv). The reaction mixture was $>_{\mathrm{H}}$ allowed to stir for $20 \mathrm{~min}$ and then the reaction was quenched with $\mathrm{MeOH}(1 \mathrm{~mL})$ and saturated aqueous sodium and potassium tartrate $(20 \mathrm{~mL})$ and then stirred vigorously for $2 \mathrm{~h}$ at $25^{\circ} \mathrm{C}$. The resulting biphasic mixture was diluted with $\mathrm{H}_{2} \mathrm{O}(20 \mathrm{~mL})$ and extracted with $\mathrm{CH}_{2} \mathrm{Cl}_{2}(3 \times 25 \mathrm{~mL})$. The combined organic extracts were dried $\left(\mathrm{Na}_{2} \mathrm{SO}_{4}\right)$, concentrated, and then purified by flash column chromatography (silica gel, EtOAc:hexanes 2:1) to afford alcohol 58 (309 mg, $0.55 \mathrm{mmol}, 92 \%$ ) as a colorless oil. 58: $R_{\mathrm{f}}=0.20$ (silica gel, EtOAc:hexanes 1:3); $[\alpha]_{\mathrm{D}}{ }^{25}=-37.4\left(\mathrm{CHCl}_{3}, c=1.00\right)$; IR (film) $v_{\max }=3464,2930,2848,1455,1425,1390,1109 \mathrm{~cm}^{-1} ;{ }^{1} \mathrm{H}$ NMR (400 MHz, $\left.\mathrm{CDCl}_{3}\right): \delta=7.69-7.64$ (m, 4 H), 7.39-7.36 (m, 6 H), 6.01-5.97 (m, 1 H), 5.73-5.70 (m, 1 H), 4.51-4.45 (m, 1 H), 4.18 (br s, 1 H), 4.15-4.11 (m, 1 H), 3.89 (br s, 1 H), 3.76 (dd, $J=11.0,4.0$ Hz, 1 H), 3.71-3.68 (m, 1 H), 3.64 (dd, $J=$ 11.0, 4.0 Hz, 1 H), 3.60-3.56 (m, 1 H), 2.29-2.23 (m, 1 H), 2.19-2.08 (m, 6 H), 1.97-1.90 (m, 3 H), 1.88-1.85 (m, 2 H), 1.49-1.42 (m, 1 H), 1.04 (s, 9 H), 0.95 (d, J = 7.0 Hz, 3 H); ${ }^{13} \mathrm{C}$ NMR (100 MHz, $\left.\mathrm{CDCl}_{3}\right): \delta=135.6,135.6,133.6,133.6,129.6,129.5,129.2,127.9,127.6,127.6,111.5,104.0,78.6$, 76.6, 76.0, 68.9, 66.2, 65.3, 36.1, 35.7, 33.9, 30.8, 26.8, 25.5, 23.3, 19.3, 15.7; HR-MS [Matrix-assisted laser desorption / ionization (MALDI)]: calcd for $\mathrm{C}_{33} \mathrm{H}_{44} \mathrm{O}_{6} \mathrm{SiNa}^{+}\left[\mathrm{M}+\mathrm{Na}^{+}\right]$: 587.2799, found 587.2801.

Aldehyde 59. To a solution of oxallyl chloride ( $0.22 \mathrm{~mL}, 2.5 \mathrm{mmol}$, 5.0 equiv) in $\mathrm{CH}_{2} \mathrm{Cl}_{2}(12 \mathrm{~mL})$ at $78^{\circ} \mathrm{C}$ was added DMSO (0.34 mmol, $5.5 \mathrm{mmol}$, 11.0 equiv). The solution was allowed to stir for $20 \mathrm{~min}$

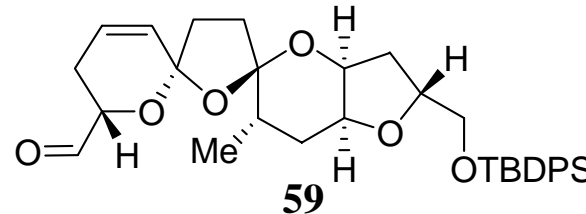

59 at $-78{ }^{\circ} \mathrm{C}$ and then a solution of alcohol $58(0.29 \mathrm{~g}, 0.51 \mathrm{mmol}, 1.0$ equiv) in $\mathrm{CH}_{2} \mathrm{Cl}_{2}(10 \mathrm{~mL})$ was added. The resulting reaction mixture was allowed to stir at $-78{ }^{\circ} \mathrm{C}$ for $1 \mathrm{~h}$, at which time $\mathrm{Et}_{3} \mathrm{~N}$ 
(1.57 mL, $11.0 \mathrm{mmol}, 22.0$ equiv) was added and the reaction mixture was allowed to warm to $0{ }^{\circ} \mathrm{C}$ over $1 \mathrm{~h}$. The reaction mixture was then quenched with saturated aqueous $\mathrm{NaCl}(30 \mathrm{~mL})$, and extracted with $\mathrm{CH}_{2} \mathrm{Cl}_{2}(3 \times 20 \mathrm{~mL})$, and the combined organic layers were dried $\left(\mathrm{Na}_{2} \mathrm{SO}_{4}\right)$, concentrated and then purified by flash column chromatography (silica gel, EtOAc:hexanes 1:1) to give aldehyde 59 (264 mg, 0.47 mmol, 92\%) as a colorless oil. 59: $R_{\mathrm{f}}=0.66$ (silica gel, EtOAc:hexanes $1: 1$ ); $[\alpha]_{\mathrm{D}}{ }^{25}=-67.1$ $\left(\mathrm{CHCl}_{3}, c=1.50\right)$; IR (film) $v_{\max }=2955,2929,2855,1739,1428,1110 \mathrm{~cm}^{-1} ;{ }^{1} \mathrm{H} \mathrm{NMR}(600 \mathrm{MHz}$, $\left.\mathrm{CDCl}_{3}\right): \delta=9.68$ (s, $\left.1 \mathrm{H}\right), 7.69-7.65$ (m, $\left.4 \mathrm{H}\right)$, 7.43-7.35 (m, $\left.6 \mathrm{H}\right), 6.02-5.99$ (m, $\left.1 \mathrm{H}\right), 5.78-5.76$ (m, 1 H), 4.49-4.45 (m, 2 H), 4.21 (br s, 1 H), 3.90 (br s, 1 H), 3.77 (dd, $J=11.0,4.0 \mathrm{~Hz}, 1 \mathrm{H}$ ), 3.64 (dd, $J=$ 11.0, 4.0 Hz, $1 \mathrm{H}), 2.28-2.04$ (m, $10 \mathrm{H}), 1.50-1.45$ (m, $1 \mathrm{H}), 1.04$ (s, $9 \mathrm{H}), 0.91$ (d, J = $7.0 \mathrm{~Hz}, 3 \mathrm{H})$; ${ }^{13} \mathrm{C}$ NMR (100 MHz, $\left.\mathrm{CDCl}_{3}\right): \delta=201.2,135.58,135.56,133.6,133.6,129.60,129.56,129.53,127.6$, 127.6, 126.9, 78.6, 76.7, 75.9, 73.1, 66.2, 36.1, 35.5, 33.9, 30.9, 26.8, 24.5, 23.2, 19.3, 15.6; HR-MS [Matrix-assisted laser desorption / ionization (MALDI)]: calcd for $\mathrm{C}_{33} \mathrm{H}_{42} \mathrm{O}_{6} \mathrm{SiNa}^{+}\left[\mathrm{M}+\mathrm{Na}^{+}\right.$]: 585.2643, found 585.2662.

Allylic acetate 61. To a solution of aldehyde 59 (460 mg, $0.677 \mathrm{mmol}, 1.0$ equiv) in $\mathrm{Et}_{2} \mathrm{O}(20 \mathrm{~mL})$ at 0 ${ }^{\circ} \mathrm{C}$ was added vinyl magnesium bromide (1.08 mL, 1.0 M in THF, $1.08 \mathrm{mmol}, 1.6$ equiv). The reaction

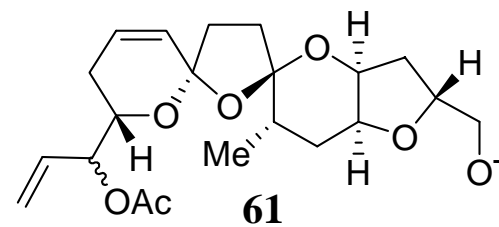
mixture was allowed to warm to $25^{\circ} \mathrm{C}$ over $1 \mathrm{~h}$ and then quenched with saturated aqueous $\mathrm{NH}_{4} \mathrm{Cl}(20 \mathrm{~mL})$. The biphasic mixture was extracted with EtOAc $(3 \times 15 \mathrm{~mL})$ and the combined organic extracts were dried $\left(\mathrm{Na}_{2} \mathrm{SO}_{4}\right)$, and concentrated, and the obtained crude allylic alcohol 60 was used in the next step without further purification. Allylic alcohol 60 (377 mg, $0.53 \mathrm{mmol}, 1.0$ equiv) was dissolved in $\mathrm{CH}_{2} \mathrm{Cl}_{2}(10 \mathrm{~mL})$ and cooled to $0{ }^{\circ} \mathrm{C}$. To the solution was added pyridine (0.42 mL, $5.3 \mathrm{mmol}, 10.0$ equiv), 4-DMAP (7.2 mg, $0.05 \mathrm{mmol}, 0.1$ equiv), and $\mathrm{Ac}_{2} \mathrm{O}$ (0.27 mL, $2.65 \mathrm{mmol}, 5.0$ equiv), and the resulting mixture was allowed to stir at $0{ }^{\circ} \mathrm{C}$ for $3 \mathrm{~h}$. When complete the reaction mixture was quenched with saturated aqueous $\mathrm{NaHCO}_{3}(10 \mathrm{~mL})$ and extracted with $\mathrm{CH}_{2} \mathrm{Cl}_{2}(3 \times 10 \mathrm{~mL})$. The combined organic extracts were dried $\left(\mathrm{Na}_{2} \mathrm{SO}_{4}\right)$, concentrated, and then purified by flash column chromatography (silica gel, EtOAc:hexanes 2:1) to afford a 2:1 ratio of allylic 
acetate 61 (240 mg, 0.38 mmol, 72\% over two steps) as a colorless oil. 61 (mixture of epimers ca 2:1, major isomer listed): $R_{\mathrm{f}}=0.67$ (silica gel, EtOAc:hexanes 1:3); IR (film) $v_{\max }=2930,1742,1455$, 1372, 1233, $1102 \mathrm{~cm}^{-1}$; ${ }^{1} \mathrm{H}$ NMR (400 MHz, $\left.\mathrm{CDCl}_{3}\right): \delta=7.69-7.64$ (m, $\left.4 \mathrm{H}\right), 7.41-7.36$ (m, $\left.6 \mathrm{H}\right)$, 5.98-5.69 (m, 3 H), 5.36-5.24 (m, 3 H), 4.49-4.46 (m, 1 H), 4.18 (br s, 1 H), 4.13 (dt, J = 10.8, 3.2 Hz, 1 H), 3.89 (br s, 1 H), 3.77 (dd, $J=11.0,4.0$ Hz, 1 H), 3.63 (dd, $J=11.0,4.0$ Hz, 1 H), 2.27-1.88 (m, 10 H), 2.06 (s, $3 \mathrm{H}), 1.50-1.42$ (m, $1 \mathrm{H}), 1.04$ (s, $9 \mathrm{H}), 0.97$ (d, J = $7.0 \mathrm{~Hz}, 3 \mathrm{H}) ;{ }^{13} \mathrm{C}$ NMR $(100 \mathrm{MHz}$, $\left.\mathrm{CDCl}_{3}\right): \delta=170.1,135.6,135.6,133.6,133.6,132.8,132.6,129.6,129.5,129.2,127.6,127.6,118.6$, 111.6, 104.1, 78.6, 76.5, 76.0, 69.4, 69.2, 66.2, 36.1, 35.6, 34.0, 30.8, 26.8, 25.0, 23.3, 21.1, 19.3, 15.8; HR-MS [Matrix-assisted laser desorption / ionization (MALDI)]: calcd for $\mathrm{C}_{37} \mathrm{H}_{48} \mathrm{O}_{7} \mathrm{SiNa}^{+}\left[\mathrm{M}+\mathrm{Na}^{+}\right]$: 655.3061, found 655.3092.

Carboxylic Acid 62. Allylic acetate 61 (33 mg, 0.043 mmol, 1.0 equiv) was dissolved in THF (3 mL) and cooled to $-78{ }^{\circ} \mathrm{C}$. To the solution was added lithium diisopropylamide $(0.065 \mathrm{~mL}, 1.0 \mathrm{M}$ in THF,

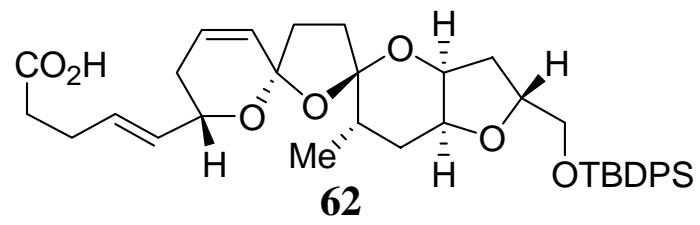
0.065 mmol, 1.5 equiv), followed by TBSCl (10.0 mL, 0.065 mmol, 1.5 equiv) and HMPA (11.8 mL, 0.065 mmol, 1.5 equiv) and the reaction mixture was allowed to warm to $25^{\circ} \mathrm{C}$ and stirred for $72 \mathrm{~h}$. When complete the reaction mixture was quenched with saturated aqueous $\mathrm{NaHCO}_{3}$ $(5 \mathrm{~mL})$ and extracted with EtOAc $(3 \times 5 \mathrm{~mL})$. The combined organic extracts were dried $\left(\mathrm{Na}_{2} \mathrm{SO}_{4}\right)$, concentrated, and then purified by flash column chromatography (silica gel, EtOAc:hexanes 3:1) to afford carboxylic acid 62 (27 mg, $0.035 \mathrm{mmol}, 82 \%$ ) as a colorless oil. 62: $R_{\mathrm{f}}=0.38$ (silica gel, EtOAc:hexanes 1:1); $[\alpha]_{\mathrm{D}}^{25}=-37.5\left(\mathrm{CHCl}_{3}, c=1.00\right)$; IR (film) $v_{\max }=3447,2930,1728,1452,1235$, $1110 \mathrm{~cm}^{-1} ;{ }^{1} \mathrm{H}$ NMR (600 MHz, $\left.\mathrm{CDCl}_{3}\right): \delta=7.67-7.63(\mathrm{~m}, 4 \mathrm{H}), 7.40-7.34(\mathrm{~m}, 6 \mathrm{H}), 5.98-5.95$ (m, 1 H), 5.72-5.66 (m, 2 H), 5.56-5.52 (m, 1 H), 4.49-4.44 (m, 1 H), 4.42-4.39 (m, 1 H), 4.17 (br s, 1 H), 3.87 (br s, 1 H), 3.75 (dd, $J=11.0,4.0 \mathrm{~Hz}, 1 \mathrm{H}$ ), 3.63 (dd, $J=11.0,4.0 \mathrm{~Hz}, 1 \mathrm{H}), 2.42-2.37$ (m, 2 H), 2.23-1.95 (m, $12 \mathrm{H}), 1.83$ (t, $J=6.0 \mathrm{~Hz}, 1 \mathrm{H}), 1.02$ (s, $9 \mathrm{H}), 0.88$ (d, $J=7.0 \mathrm{~Hz}, 3 \mathrm{H}) ;{ }^{13} \mathrm{C}$ NMR $(150$ $\left.\mathrm{MHz}, \mathrm{CDCl}_{3}\right): \delta=179.3,135.6,135.5,133.6,133.6,131.1,130.2,129.6,129.5,128.4,127.6,127.6$, 125.2, 111.5, 104.1, 78.6, 76.5, 76.0, 68.6, 66.2, 36.1, 35.7, 33.9, 33.5, 30.9, 29.9, 27.5, 26.7, 23.3, 19.2, 
15.6; HR-MS [Matrix-assisted laser desorption / ionization (MALDI)]: calcd for $\mathrm{C}_{37} \mathrm{H}_{48} \mathrm{O}_{7} \mathrm{SiNa}^{+}[\mathrm{M}+$ $\mathrm{Na}^{+}$]: 655.3061, found 655.3072 .

Methyl Ester 63. To a solution of carboxylic acid 62 (27 mg, $0.035 \mathrm{mmol}, 1.0$ equiv) in $\mathrm{CH}_{2} \mathrm{Cl}_{2}(2 \mathrm{~mL})$ at $0{ }^{\circ} \mathrm{C}$ was added DCC (8.7 mg, $0.042 \mathrm{mmol}, 1.2$ equiv), 4-DMAP (0.5 mg, $0.004 \mathrm{mmol}, 0.1$ equiv),

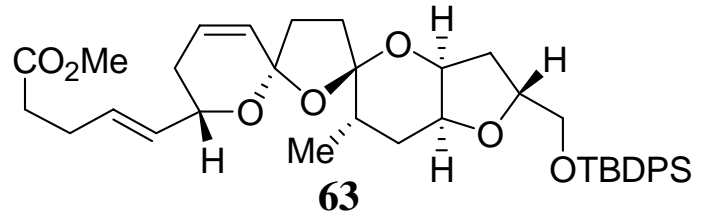
and $\mathrm{MeOH}(15 \mu \mathrm{L}, 0.35 \mathrm{mmol}, 10$ equiv). The reaction mixture was allowed to warm to $25{ }^{\circ} \mathrm{C}$ over $2 \mathrm{~h}$ then quenched with $\mathrm{H}_{2} \mathrm{O}$ (3 mL). The biphasic mixture was extracted with $\mathrm{CH}_{2} \mathrm{Cl}_{2}(3 \times 4 \mathrm{~mL})$ and the combined organic extracts were dried $\left(\mathrm{Na}_{2} \mathrm{SO}_{4}\right)$, concentrated, and then purified by flash column chromatography (silica gel, EtOAc:hexanes 1:1) to afford methyl ester 63 (20 mg, $0.030 \mathrm{mmol}$, 86\%) as a colorless oil. 63: $R_{\mathrm{f}}=0.39$ (silica gel, EtOAc:hexanes 1:3); $[\alpha]_{\mathrm{D}}{ }^{25}=-23.3\left(\mathrm{CHCl}_{3}, \mathrm{c}=0.40\right)$; IR (film) $v_{\max }=2928,1738,1633,1428,1231,1111 \mathrm{~cm}^{-1} ;{ }^{1} \mathrm{H}$ NMR (500 MHz, $\mathrm{CDCl}_{3}$ ): $\delta=7.69-7.65$ (m, 4 H), 7.42-7.35 (m, $6 \mathrm{H}$ ), 5.98 (ddd, $J=10.0,5.5,2.0 \mathrm{~Hz}, 1 \mathrm{H}$ ), 5.72-5.68 (m, 2 H), 5.54 (dd, $J$ = 15.5, 5.5 Hz, 1 H), 4.47 (ddd, $J$ = 12.0, 10.0, 4.0 Hz, 1 H), 4.43 (m, 1 H), 4.18 (br s, 1 H), 3.89 (br s, 1 H), 3.76 (dd, $J$ = 11.0, 4.0 Hz, 1 H), 3.66 (s, 3 H), 3.64 (dd, $J$ = 11.0, $4.0 \mathrm{~Hz}, 1 \mathrm{H}), 2.40-2.34$ (m, $4 \mathrm{H}), 2.26-2.18(\mathrm{~m}, 1 \mathrm{H}), 2.17-2.07$ (m, $4 \mathrm{H}), 2.06-1.95$ (m, $5 \mathrm{H})$, 1.48-1.42 (m, $1 \mathrm{H}), 1.03$ (s, $9 \mathrm{H}), 0.89$ (d, $J=7.0 \mathrm{~Hz}, 3 \mathrm{H}) ;{ }^{13} \mathrm{C}$ NMR $\left(125 \mathrm{MHz}, \mathrm{CDCl}_{3}\right.$ ): $\delta=173.3$, 135.6, 135.5, 133.6, 133.5, 131.1, 130.1, 130.0, 129.6, 129.5, 129.1, 128.3, 127.6, 78.6, 76.5, 76.0, 68.5, 66.2, 36.1, 35.7, 33.9, 33.5, 30.9, 29.9, 29.6, 27.6, 26.8, 23.2, 19.2, 15.5; HR-MS [Matrix-assisted laser desorption / ionization (MALDI)]: calcd for $\mathrm{C}_{38} \mathrm{H}_{50} \mathrm{O}_{7} \mathrm{SiNa}^{+}\left[\mathrm{M}+\mathrm{Na}^{+}\right]$: 669.3218, found 669.3220.

Reduced Product 64. To a solution of methyl ester 63 (20 mg, $0.030 \mathrm{mmol}, 1.0$ equiv) in THF (2 mL) at $-78{ }^{\circ} \mathrm{C}$ was added a solution of superhydride ( $0.15 \mathrm{~mL}, 1.0 \mathrm{M}$ in THF, $0.15 \mathrm{mmol}$, 5.0 equiv). The

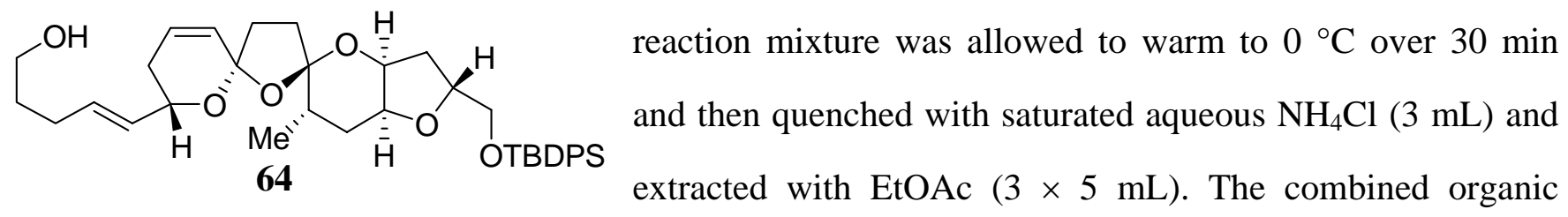
extracts were dried $\left(\mathrm{Na}_{2} \mathrm{SO}_{4}\right)$, concentrated, and then purified by flash column chromatography (silica 
gel, EtOAc:hexanes 3:1) to afford reduced product 64 (17.8 mg, $0.029 \mathrm{mmol}, 96 \%)$ as a colorless oil. 64: $R_{\mathrm{f}}=0.11$ (silica gel, EtOAc:hexanes 1:3); $[\alpha]_{\mathrm{D}}{ }^{25}=-45.5\left(\mathrm{CHCl}_{3}, c=3.50\right)$; IR (film) $v_{\max }=3486$, 2930, 2858, 1325, $1110 \mathrm{~cm}^{-1}$; ${ }^{1} \mathrm{H}$ NMR (400 MHz, $\mathrm{CDCl}_{3}$ ): $\delta=7.69-7.65$ (m, $4 \mathrm{H}$ ), $7.42-7.35$ (m, 6 H), 6.00-5.95 (m, 1 H), 5.72-5.68 (m, 2 H), 5.56-5.51 (m, 1 H), 4.47-4.38 (m, 2 H), 4.17 (br s, 1 H), 3.84 (br s, 1 H), 3.80-3.72 (m, 3 H), 3.62 (dd, $J=11.0,4.0$ Hz, 1 H), 2.24-1.91 (m, 14 H), $1.63-1.57$ (m, $2 \mathrm{H}), 1.44-1.38$ (m, $1 \mathrm{H}), 1.03$ (s, $9 \mathrm{H}), 0.89$ (d, $J=7.0 \mathrm{~Hz}, 3 \mathrm{H}) ;{ }^{13} \mathrm{C}$ NMR (100 MHz, $\left.\mathrm{CDCl}_{3}\right): \delta=$ 135.57, 135.55, 133.6, 133.6, 132.0, 131.8, 130.5, 129.6, 129.5, 128.4, 127.6, 127.6, 111.5, 104.1, 78.3, 76.5, 76.0, 68.8, 66.2, 62.5, 36.1, 35.7, 34.0, 32.0, 30.9, 30.0, 28.7, 26.8, 23.3, 19.2, 15.6; HR-MS [Matrix-assisted laser desorption / ionization (MALDI)]: calcd for $\mathrm{C}_{37} \mathrm{H}_{50} \mathrm{O}_{6} \mathrm{SiNa}^{+}\left[\mathrm{M}+\mathrm{Na}^{+}\right.$]: 641.3269, found 641.3246.

Pivaloate 65. Reduced product 64 (17.8 mg, 0.029 mmol, 1.0 equiv) was dissolved in $\mathrm{CH}_{2} \mathrm{Cl}_{2}(2 \mathrm{~mL})$ and cooled to $0{ }^{\circ} \mathrm{C}$. To the solution was added pyridine (0.023 mL, $0.29 \mathrm{mmol}, 10.0$ equiv), 4-DMAP

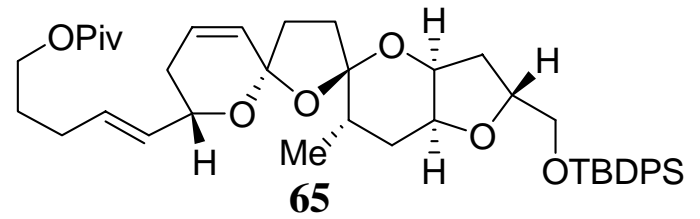
(3.5 mg, 0.029 mmol, 1.0 equiv), and PivCl (0.011 mL, 0.087 mmol, 3.0 equiv). The reaction mixture was allowed to warm to $25{ }^{\circ} \mathrm{C}$ over $3 \mathrm{~h}$ and then concentrated, and the resulting residue was purified by flash column chromatography (silica gel, EtOAc:hexanes 1:1) to afford pivaloate 65 (19.4 mg, $0.028 \mathrm{mmol}, 95 \%)$ as a colorless oil. 65: $R_{\mathrm{f}}=0.57$ (silica gel, EtOAc:hexanes 1:3); $[\alpha]_{\mathrm{D}}{ }^{25}=-37.5\left(\mathrm{CHCl}_{3}, c=1.60\right)$; IR (film) $v_{\max }=2932,2859,1727,1458,1285,1155 \mathrm{~cm}^{-1} ;{ }^{1} \mathrm{H}$ NMR (400 MHz, $\mathrm{CDCl}_{3}$ ): $\delta=7.69-7.64$ (m, $\left.4 \mathrm{H}\right), 7.42-7.35$ (m, $\left.6 \mathrm{H}\right), 6.00-5.96$ (m, $\left.1 \mathrm{H}\right)$, 5.74-5.65 (m, 2 H), 5.52 (dd, $J=16.0,6.0$ Hz, 1 H), 4.50-4.41 (m, 2 H), 4.19 (br s, 1 H), 4.04 (t, J = 6.8 Hz, 2 H), 3.89 (br s, 1 H), 3.77 (dd, $J=11.0,4.0$ Hz, 1 H), 3.64 (dd, $J=11.0,4.0$ Hz, 1 H), 2.26-1.95 (m, 11 H), 1.74-1.67 (m, 2 H), 1.49-1.41 (m, $1 \mathrm{H}), 1.19$ (s, $9 \mathrm{H}), 1.03$ (s, $9 \mathrm{H}), 0.90$ (d, $J=7.0 \mathrm{~Hz}, 3 \mathrm{H}) ;{ }^{13} \mathrm{C}$ NMR $\left(100 \mathrm{MHz}, \mathrm{CDCl}_{3}\right): \delta=178.5,135.58,135.56,133.6,133.6,131.1,130.9,129.6,129.5,129.2,128.4$, 127.6, 127.6, 111.5, 104.1, 78.6, 76.6, 76.0, 68.8, 66.2, 63.6, 40.2, 38.7, 36.2, 35.7, 34.0, 31.6, 30.9, 30.0, 28.7, 28.0, 27.2, 23.3, 15.6; HR-MS [Matrix-assisted laser desorption / ionization (MALDI)]: calcd for $\mathrm{C}_{42} \mathrm{H}_{58} \mathrm{O}_{7} \mathrm{SiNa}^{+}\left[\mathrm{M}+\mathrm{Na}^{+}\right]$: 725.3844, found 725.3853 . 
TES ether 67. To a solution of epimerized product 53 ( $0.65 \mathrm{~g}, 1.0 \mathrm{mmol}, 1.0$ equiv) in $\mathrm{CH}_{2} \mathrm{Cl}_{2}(5 \mathrm{~mL})$ at $-78{ }^{\circ} \mathrm{C}$ was added imidazole ( $0.20 \mathrm{~g}, 3.0 \mathrm{mmol}$, 3.0 equiv), 4-DMAP ( $15 \mathrm{mg}, 0.1 \mathrm{mmol}, 0.1$ equiv), and

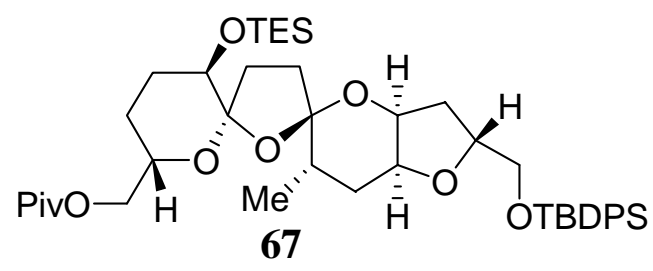

TESCl (250 $\mu \mathrm{L}, 1.5 \mathrm{mmol}, 1.5$ equiv). The reaction mixture was warmed to $0{ }^{\circ} \mathrm{C}$ over $10 \mathrm{~h}$ and then quenched with saturated aqueous $\mathrm{NaHCO}_{3}(10 \mathrm{ml})$. The biphasic mixture was extracted with $\mathrm{CH}_{2} \mathrm{Cl}_{2}(3 \times 10 \mathrm{~mL})$ and the combined organic layers were dried $\left(\mathrm{Na}_{2} \mathrm{SO}_{4}\right)$, concentrated, and then purified by flash column chromatography (silica gel, hexanes:EtOAc 10:1 $\rightarrow$ 5:1) to afford TES ether $\mathbf{6 7}(0.70 \mathrm{~g}, 0.92 \mathrm{mmol}, 92 \%)$ as a colorless oil. 67: $R_{\mathrm{f}}=$ 0.45 (silica gel, EtOAc:hexanes 1:5); $[\alpha]_{\mathrm{D}}^{34}=-8.4\left(\mathrm{CH}_{3} \mathrm{OH}, c=2.0\right)$; IR (film) $v_{\max }=2956,2875$, 1730, 1459, 1428, 1321, 1283, 1238, 1153, 1114, 1073, 1019, 988, 827, 739, $703 \mathrm{~cm}^{-1}$; ${ }^{1} \mathrm{H}$ NMR (600 $\left.\mathrm{MHz}, \mathrm{CDCl}_{3}\right): \delta=7.80-7.72(\mathrm{~m}, 4 \mathrm{H}), 7.52-7.40$ (m, $\left.6 \mathrm{H}\right), 4.62-4.59$ (m, $\left.1 \mathrm{H}\right), 4.24$ (br s, $1 \mathrm{H}$ ), 4.19-4.12 (m, 1 H), 4.05-4.00 (m, 2 H), 3.96 (br s, 1 H), 3.84 (dd, $J$ = 13.2, 4.8, 1 H), 3.75-3.65 (m, 2 H), 2.50-2.30 (m, 2 H), 2.30-1.60 (m, 9 H), 1.60-1.40 (m, 2 H), 1.27 (s, 9 H), $1.16-0.62$ (m, $27 \mathrm{H}) ;{ }^{13} \mathrm{C}$ NMR (125 MHz, $\left.\mathrm{CDCl}_{3}\right): \delta=178.5,135.6,133.6,129.5,127.6,112.2,108.1,78.4,76.5,75.8,69.7$, 67.6, 66.9, 66.3, 38.7, 35.6, 34.1, 31.0, 28.6, 27.7, 27.2, 26.8, 22.4, 19.2, 16.1, 6.9, 6.5, 5.8, 5.0; HR-MS [electrospray ionization (ESI)]: calcd for $\mathrm{C}_{44} \mathrm{H}_{69} \mathrm{O}_{8} \mathrm{Si}_{2}{ }^{+}\left[\mathrm{M}+\mathrm{H}^{+}\right]$: 781.4525, found 781.4516.

Primary Alcohol 68. To a solution of TES ether 67 (0.70 g, $0.90 \mathrm{mmol}, 1.0$ equiv) in $\mathrm{CH}_{2} \mathrm{Cl}_{2}(15 \mathrm{~mL})$ at $-78{ }^{\circ} \mathrm{C}$ was added a solution of DIBAL-H (2.7 mL, 1.0 M in toluene, $2.7 \mathrm{mmol}$, 3.0 equiv) over 10

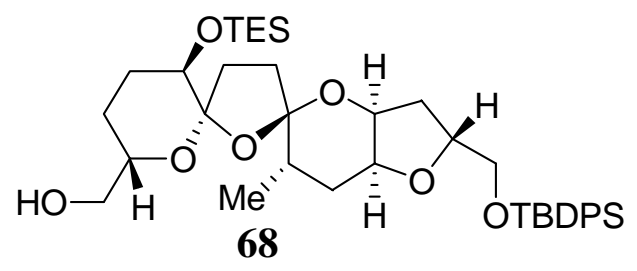
$\min$. The reaction mixture was stirred at $-78^{\circ} \mathrm{C}$ for $1 \mathrm{~h}$ and then quenched with $\mathrm{MeOH}(1.0 \mathrm{~mL})$ and allowed to warm to $25^{\circ} \mathrm{C}$. A solution of saturated aqueous sodium and potassium tartrate (30 $\mathrm{mL}$ ) was then added and the mixture was vigorously stirred at 25

${ }^{\circ} \mathrm{C}$ for $1 \mathrm{~h}$. The biphasic mixture was extracted with $\mathrm{CH}_{2} \mathrm{Cl}_{2}(3 \times 20 \mathrm{~mL})$ and the combined organic extracts were dried $\left(\mathrm{Na}_{2} \mathrm{SO}_{4}\right)$, concentrated, and then purified by flash column chromatography (silica gel, hexanes:EtOAc 10:1 $\rightarrow$ 1:1) to afford primary alcohol $68(0.54 \mathrm{~g}, 0.78 \mathrm{mmol}, 87 \%)$ as a colorless 
oil. 68: $R_{\mathrm{f}}=0.15$ (silica gel, EtOAc:hexane 1:5); $[\alpha]_{\mathrm{D}}^{33}=-14.8(\mathrm{MeOH}, c=2.50)$; IR (film) $v_{\max }=$ 3564, 3483, 2956, 2873, 2359, 1732, 1456, 1384, 1273, 1115, 1073, 1020, 985, 826, 742, $704 \mathrm{~cm}^{-1} ;{ }^{1} \mathrm{H}$ NMR (500 MHz, $\mathrm{CDCl}_{3}$ ): $\delta=7.75-7.65$ (m, 4 H), 7.45-7.35 (m, 6 H), 4.45-4.39 (m, 1 H), 4.13 (br s, 1 H), 3.94-3.91 (m, 1 H), 3.82 (br s, 1 H), 3.74 (dd, $J=11.0,3.7,1$ H), 3.68-3.58 (m, 2 H), 3.55-3.52 (m, $1 \mathrm{H}), 3.42-3.38$ (m, $1 \mathrm{H}), 2.40-2.37$ (m, $1 \mathrm{H}), 2.21-2.18$ (m, $1 \mathrm{H}), 2.15-1.40$ (m, $11 \mathrm{H}), 1.10-0.95$ (m, $18 \mathrm{H}), 0.90$ (d, $J=6.6 \mathrm{~Hz}, 3 \mathrm{H}), 0.75-0.55$ (m, $6 \mathrm{H}) ;{ }^{13} \mathrm{C} \mathrm{NMR}\left(125 \mathrm{MHz}, \mathrm{CD}_{2} \mathrm{Cl}_{2}\right): \delta=136.2,134.1$, 133.8, 131.5, 130.5, 129.2, 128.9, 112.7, 108.6, 78.9, 76.7, 76.2, 70.6, 70.2, 68.4, 66.0, 39.2, 36.1, 34.3, 31.6, 30.8, 29.3, 29.1, 27.1, 27.0, 24.1, 23.4, 22.5, 19.5, 16.1, 14.2, 11.1, 7.1, 5.3; HR-MS [electrospray ionization (ESI)]: calcd for $\mathrm{C}_{39} \mathrm{H}_{60} \mathrm{O}_{7} \mathrm{Si}_{2} \mathrm{Na}^{+}\left[\mathrm{M}+\mathrm{Na}^{+}\right]$: 719.3770, found 719.3776.

Alkene 70. To a solution of DMSO (0.66 mL, $9.4 \mathrm{mmol}, 10$ equiv) in $\mathrm{CH}_{2} \mathrm{Cl}_{2}(15 \mathrm{~mL})$ at $-78{ }^{\circ} \mathrm{C}$ was added oxallyl chloride $\left(0.40 \mathrm{~mL}, 4.7 \mathrm{mmol}, 5.0\right.$ equiv). The solution was stirred at $-78{ }^{\circ} \mathrm{C}$ for $15 \mathrm{~min}$ $=C_{\mathrm{H}}$ and then a solution of primary alcohol 68 (0.64 g, $0.94 \mathrm{mmol}, 1.0$ equiv) in $\mathrm{CH}_{2} \mathrm{Cl}_{2}(1.5 \mathrm{~mL})$ was added slowly. The reaction mixture was maintained at $-78^{\circ} \mathrm{C}$ for $1 \mathrm{~h}$, then $\mathrm{Et}_{3} \mathrm{~N}(2.0 \mathrm{~mL}, 14.0 \mathrm{mmol}, 15$ equiv) was added and the solution was allowed to warm to $0{ }^{\circ} \mathrm{C}$ over $1 \mathrm{~h}$. The reaction mixture was then quenched with saturated aqueous $\mathrm{NaCl}(20 \mathrm{ml})$ and extracted with $\mathrm{CH}_{2} \mathrm{Cl}_{2}(3 \times 20 \mathrm{~mL})$. The combined organic layers were dried $\left(\mathrm{Na}_{2} \mathrm{SO}_{4}\right)$ and concentrated to provide crude aldehyde 69 which was carried on without any further purification. Methyltriphenylphosphonium bromide (0.31 g, 0.89 mmol, 6.0 equiv) was dissolved in THF $(10 \mathrm{~mL})$ and cooled to $-78^{\circ} \mathrm{C}$. To this solution was added $n$-BuLi $(0.48 \mathrm{~mL}, 1.6$ $\mathrm{M}$ in THF, $0.75 \mathrm{mmol}, 5.0$ equiv) and the resulting mixture was allowed to warm to $0{ }^{\circ} \mathrm{C}$ over $1 \mathrm{~h}$. The solution was then re-cooled to $-78{ }^{\circ} \mathrm{C}$ and a solution of crude aldehyde 69 (0.64 g, 0.94 mmol, 1.0 equiv) in THF (10 mL) was added. The resulting reaction mixture was allowed to warm to $0{ }^{\circ} \mathrm{C}$ over 30 min and was then quenched with saturated aqueous $\mathrm{NH}_{4} \mathrm{Cl}(15 \mathrm{~mL})$. The biphasic mixture was extracted with EtOAc $(3 \times 15 \mathrm{~mL})$ and the combined organic extracts were dried $\left(\mathrm{MgSO}_{4}\right)$, concentrated, and then purified by flash column chromatography (silica gel, hexanes:EtOAc 20:1 $\rightarrow$ 10:1) to afford alkene 70 (45 mg, $0.67 \mathrm{mmol}, 71 \%$ over two steps) as colorless oil. 70: $R_{\mathrm{f}}=0.73$ (silica gel, EtOAc:hexane 1:10); 
$[\alpha]_{\mathrm{D}}^{33}=-8.80(\mathrm{MeOH}, c=2.5)$; IR (film) $v_{\max }=2955,2929,2874,2835,1458,1424,1379,1363$, 1319, 1243, 1152, 1113, 1072, 1015, 974, 855, 819, 738, 702, $\left.614 \mathrm{~cm}^{-1} ;{ }^{1} \mathrm{H} \mathrm{NMR} 400 \mathrm{MHz}, \mathrm{CDCl}_{3}\right): \delta$ = 7.73-7.63 (m, 4 H), 7.45-7.32 (m, 6 H), 5.83-5.77 (m, $1 \mathrm{H}), 5.18$ (d, $J=17.3 \mathrm{~Hz}, 1 \mathrm{H}), 5.10(\mathrm{~d}, J=$ 10.6 Hz, 1 H), 4.55-4.53 (m, 1 H), 4.37-4.34 (m, 1 H), 4.17 (br s, 1 H), 3.88 (br s, 1 H), 3.77 (dd, $J=$ 10.6, 4.1 Hz, 1 H), 3.70-3.55 (m, 2 H), 2.45-1.30 (m, 13 H), 1.10-0.95 (m, 18 H), 0.89 (d, J = 6.7 Hz, 1 $\mathrm{H}), 0.75-0.55(\mathrm{~m}, 6 \mathrm{H}) ;{ }^{13} \mathrm{C}$ NMR (100 MHz, $\left.\mathrm{CD}_{2} \mathrm{Cl}_{2}\right): \delta=139.2,135.9,134.1,129.9,127.9,114.7$, 112.7, 108.6, 79.0, 76.7, 76.2, 70.8, 70.0, 66.9, 36.1, 34.4, 31.9, 31.6, 31.3, 29.7, 27.3, 27.0, 23.0, 22.4, 19.5, 15.9, 14.3, 7.1, 5.3; HR-MS [electrospray ionization (ESI)]: calcd for $\mathrm{C}_{40} \mathrm{H}_{61} \mathrm{O}_{6} \mathrm{Si}_{2} \mathrm{H}^{+}\left[\mathrm{M}+\mathrm{H}^{+}\right]$: 693.4001, found 693.3997.

Metathesis product 73. To a solution of alkene 70 (5.2 g, $7.8 \mathrm{mmol}, 1.0$ equiv) in $\mathrm{CH}_{2} \mathrm{Cl}_{2}$ (50 mL) at $25{ }^{\circ} \mathrm{C}$ was added olefin 72 (4.0 g, 23.4 mmol, 3.0 equiv) and Grubbs' second generation catalyst 71

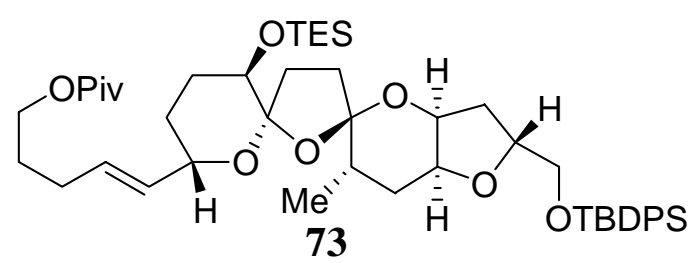

73 (0.46 g, $0.55 \mathrm{mmol}, 0.07$ equiv). The reaction mixture was heated at $40{ }^{\circ} \mathrm{C}$ for $12 \mathrm{~h}$, then cooled to $25^{\circ} \mathrm{C}$, and concentrated, and the resulting residue was purified by flash column chromatography (silica gel, hexanes:EtOAc 20:1 $\rightarrow$

10:1) to afford metathesis product 73 (4.2 g, $5.1 \mathrm{mmol}, 65 \%)$ as a colorless oil along with recovered alkene 70 (1.8 g, $2.73 \mathrm{mmol}$, 35\%), which was re-subjected to the reaction conditions. 73: $R_{\mathrm{f}}=0.77$ (silica gel, EtOAc:hexanes 1:5); $[\alpha]_{\mathrm{D}}^{33}=-8.4(\mathrm{MeOH}, c=0.7)$; IR (film) $v_{\max }=3436,2928,2869$, 1727, 1458, 1428, 1384, 1359, 1319, 1284, 1234, 1154, 1115, 1069, 1014, 980, 920, 861, 821, 736, 701 $\mathrm{cm}^{-1} ;{ }^{1} \mathrm{H}$ NMR (500 MHz, $\mathrm{CDCl}_{3}$ ): $\delta=7.72-7.62$ (m, $\left.4 \mathrm{H}\right), 7.44-7.33(\mathrm{~m}, 6 \mathrm{H}), 5.62-5.57$ (m, $\left.1 \mathrm{H}\right)$, 5.40 (dd, $J=12.8,5.1 \mathrm{~Hz}, 1 \mathrm{H}), 4.55-4.51$ (m, $1 \mathrm{H}), 4.32-4.28$ (m, $1 \mathrm{H}), 4.16$ (br s, $1 \mathrm{H}), 4.0$ (t, $J=5.5$ Hz, 2 H), 3.88 (br s, 1 H), 3.77 (dd, $J=8.8,2.9$ Hz, 1 H), 3.70-3.58 (m, 2 H), 2.45-1.40 (m, 17 H), 1.2 (s, $9 \mathrm{H}), 1.08-0.95,(\mathrm{~m}, 18 \mathrm{H}), 0.73-0.56$ (m, $6 \mathrm{H}) ;{ }^{13} \mathrm{C} \mathrm{NMR}\left(100 \mathrm{MHz}, \mathrm{CDCl}_{3}\right): \delta=178.6,135.6$, 133.7, 131.2, 130.6, 129.5, 127.6, 112.3, 108.3, 78.4, 76.6, 75.9, 70.2, 69.7, 66.3, 63.7, 35.6, 34.2, 31.2, 31.0, 29.2, 28.7, 28.0, 27.2, 26.8, 22.2, 19.3, 15.7, 6.9, 5.0; HR-MS [electrospray ionization (ESI)]: calcd for $\mathrm{C}_{48} \mathrm{H}_{74} \mathrm{O}_{8} \mathrm{Si}_{2} \mathrm{Na}^{+}\left[\mathrm{M}+\mathrm{Na}^{+}\right]$: 857.4814, found 857.4804. 
Alcohol 74. Metathesis product 73 (5.5g, $6.6 \mathrm{mmol}, 1.0$ equiv) was dissolved in a mixture of pyridine:THF $(1: 1,30 \mathrm{ml})$ and cooled to $0^{\circ} \mathrm{C}$. $\mathrm{HF} \bullet$ pyridine $(5 \mathrm{ml})$ was added dropwise and the reaction

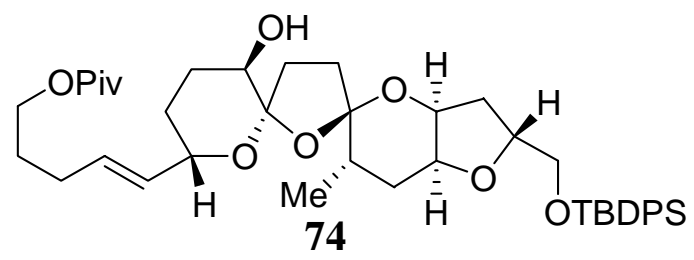
mixture was stirred at $0{ }^{\circ} \mathrm{C}$ for $5 \mathrm{~h}$ and then quenched by the slow addition of saturated aqueous $\mathrm{NaHCO}_{3}(50 \mathrm{ml})$. The biphasic mixture was extracted with EtOAc $(3 \times 40 \mathrm{ml})$ and the combined organic extracts were dried $\left(\mathrm{Na}_{2} \mathrm{SO}_{4}\right)$, concentrated, and then purified by flash column chromatography (silica gel, hexanes:EtOAc 20:1 $\rightarrow$ 5:1) to afford alcohol 74 (4.5 g, $6.2 \mathrm{mmol}, 94 \%)$ as a colorless oil. 74: $R_{\mathrm{f}}=0.32$ (silica gel, hexane:Et $\mathrm{E}_{2} \mathrm{O}$ $1: 1) ;[\alpha]_{\mathrm{D}}^{33}=-9.9(\mathrm{MeOH}, c=2.70) ;$ IR (film) $v_{\max }=3516,2931,2857,1727,1461,1428,1384,1284$, 1152, 1111, 1073, $1019 \mathrm{~cm}^{-1}$; ${ }^{1} \mathrm{H}$ NMR (500 MHz, $\mathrm{CD}_{2} \mathrm{Cl}_{2}$ ): $\delta=7.75-7.65$ (m, $\left.4 \mathrm{H}\right), 7.48-7.35$ (m, 6 H), 5.65-5.61 (m, $1 \mathrm{H}), 5.40$ (dd, $J=15.4,6.6 \mathrm{~Hz}, 1 \mathrm{H}), 4.40-4.37$ ( m, $1 \mathrm{H}), 4.27-4.21$ (m, $1 \mathrm{H}$ ), 4.17 (br s, $1 \mathrm{H}$ ), 4.00 (t, $J=6.3 \mathrm{~Hz}, 2 \mathrm{H}$ ), 3.85 (br s, $1 \mathrm{H}$ ), 3.73 (dd, $J=10.6,3.7 \mathrm{~Hz}, 1 \mathrm{H}$ ), 3.64 (dd, $J=10.7$, $4.1 \mathrm{~Hz}, 1 \mathrm{H}), 3.42-3.37$ (m, 1 H), 2.72 (d, $J=10.7$ Hz, 1 H), 2.50-1.35 (m, 17 H), 1.25-0.98 (s, 18 H), $0.92(\mathrm{~d}, J=6.9 \mathrm{~Hz}, 3 \mathrm{H}) ;{ }^{13} \mathrm{C}$ NMR $\left(125 \mathrm{MHz}, \mathrm{CD}_{2} \mathrm{Cl}_{2}\right): \delta=178.6,135.9,134.0,131.6,131.2,129.9$, 127.9, 112.1, 109.3, 79.0, 76.8, 76.1, 71.7, 68.5, 66.6, 63.9, 38.9, 36.4, 33.9, 31.7, 31.3, 30.9, 30.4, 29.9, 28.9, 28.4, 27.3, 26.9, 23.1, 19.5, 15.7; HR-MS [electrospray ionization (ESI)]: calcd for $\mathrm{C}_{42} \mathrm{H}_{60} \mathrm{O}_{8} \mathrm{SiNa}^{+}$ $\left[\mathrm{M}+\mathrm{Na}^{+}\right]:$743.3949, found 743.3941.

Ketone 75. To a solution of oxallyl chloride (0.22 mL, $2.5 \mathrm{mmol}, 5.0$ equiv) in $\mathrm{CH}_{2} \mathrm{Cl}_{2}(12 \mathrm{~mL})$ at -78 ${ }^{\circ} \mathrm{C}$ was added DMSO (0.34 mmol, $5.5 \mathrm{mmol}, 11.0$ equiv). The solution was allowed to stir for $20 \mathrm{~min}$ at $\mathrm{N}_{\mathrm{H}}^{\text {OPiv }}$ $-78{ }^{\circ} \mathrm{C}$ and then a solution of alcohol 74 (0.37 g, $0.51 \mathrm{mmol}$, 1.0 equiv) in $\mathrm{CH}_{2} \mathrm{Cl}_{2}(10 \mathrm{~mL})$ was added and the resulting mixture was allowed to stir at $-78^{\circ} \mathrm{C}$ for $1 \mathrm{~h}$, at which time $\mathrm{Et}_{3} \mathrm{~N}$ (1.57 mL, $11.0 \mathrm{mmol}, 22.0$ equiv) was added. The reaction mixture was allowed to warm to $0{ }^{\circ} \mathrm{C}$ over $1 \mathrm{~h}$ then quenched with saturated aqueous $\mathrm{NaCl}(30 \mathrm{~mL})$. The biphasic mixture was extracted with $\mathrm{CH}_{2} \mathrm{Cl}_{2}(3 \times 20 \mathrm{~mL})$ and the combined organic layers were dried $\left(\mathrm{Na}_{2} \mathrm{SO}_{4}\right)$, concentrated, and then 
purified by flash column chromatography (silica gel, EtOAc:hexanes 1:1) to give ketone 75 ( 0.35 g, 0.48 mmol, 95\%) as a colorless oil. 75: $R_{\mathrm{f}}=0.41$ (silica gel, hexane:Et $\left.{ }_{2} \mathrm{O} 1: 1\right) ;[\alpha]_{\mathrm{D}}^{33}=-6.6(\mathrm{MeOH}, c=$ 9.1); IR (film) $v_{\max }=3442,2958,2930,2857,1730,1644,1462,1428,1384,1361,1323,1284,1245$, 1153, 1112, 1073, 1022, 986, 871, 823, 741, 703, 612, $506 \mathrm{~cm}^{-1} ;{ }^{1} \mathrm{H}$ NMR $\left(500 \mathrm{MHz}, \mathrm{CDCl}_{3}\right): \delta=$ 7.75-7.60 (m, 4 H), 7.50-7.35 (m, 6 H), 5.73-5.68 (m, 1 H), 5.45 (dd, J = 15.4, 6.3 Hz, 1 H), 4.79-4.74 (m, 1 H), 4.39-4.34 (m, 1 H), 4.14 (br s, 1 H), 4.00 (t, $J=6.3$ Hz, 2 H), 3.85-3.80 (m, 1 H), 3.71 (dd, $J$ = 10.7, $4.0 \mathrm{~Hz}, 1 \mathrm{H}), 3.63(\mathrm{dd}, J=10.7,4.0 \mathrm{~Hz}, 1 \mathrm{H}), 2.95-2.89(\mathrm{~m}, 1 \mathrm{H}), 2.71-2.66(\mathrm{~m}, 1 \mathrm{H})$, 2.48-2.42 (m, 1 H), 2.25-1.80 (m, 10 H), 1.75-1.62 (m, 3 H), 1.46-1.40 (m, 1 H), 1.25 (s, 9 H), 1.00 (s, $9 \mathrm{H}), 0.93$ (d, $J=6.9 \mathrm{~Hz}, 3 \mathrm{H}$ ); ${ }^{13} \mathrm{C}$ NMR (125 MHz, $\left.\mathrm{CD}_{2} \mathrm{Cl}_{2}\right): \delta=202.1,178.6,135.9,134.1,132.1$, 130.2, 129.9, 128.0, 113.5, 107.3, 79.1, 76.9, 76.0, 71.1, 66.7, 63.8, 38.9, 36.6, 36.2, 34.1, 31.3, 29.6, 28.9, 28.4, 27.3, 27.0, 22.9, 19.5, 15.8; HR-MS [electrospray ionization (ESI)]: calcd for $\mathrm{C}_{42} \mathrm{H}_{58} \mathrm{O}_{8} \mathrm{SiNa}^{+}$ $\left[\mathrm{M}+\mathrm{Na}^{+}\right]:$741.3793, found 741.3786 .

Enol triflate 76: To a solution of ketone 75 ( 0.17 g, 0.23 mmol, 1.0 equiv) and Comin's reagent 55 (472 mg, $1.15 \mathrm{mmol}$, 5.0 equiv) in THF $(10 \mathrm{~mL})$ at $-78{ }^{\circ} \mathrm{C}$ was added KHMDS (2.17 $\mathrm{mL}, 0.5 \mathrm{M}$ in toluene,

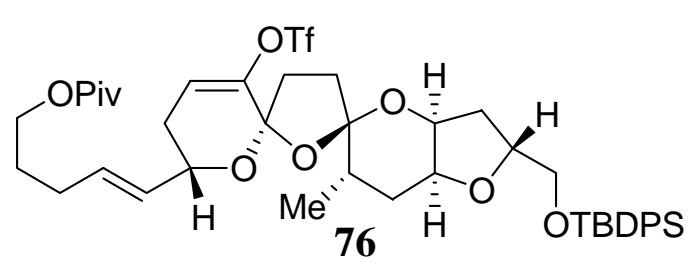
$1.04 \mathrm{mmol}, 4.5$ equiv). The reaction mixture was allowed to stir for $1 \mathrm{~h}$ at $-78{ }^{\circ} \mathrm{C}$ and then quenched with saturated aqueous $\mathrm{NaHCO}_{3}(20 \mathrm{~mL})$. The biphasic mixture was extracted with EtOAc $(3 \times 15 \mathrm{~mL})$ and the combined organic extracts were dried $\left(\mathrm{Na}_{2} \mathrm{SO}_{4}\right)$, concentrated, and then purified by flash column chromatography (silica gel, EtOAc:hexanes 1:1) to afford enol triflate $76(0.18 \mathrm{~g}, 0.22 \mathrm{mmol}, 94 \%)$ as a colorless oil. 76: $R_{\mathrm{f}}=$ 0.62 (silica gel, hexanes: $\left.\mathrm{Et}_{2} \mathrm{O} 1: 1\right) ;[\alpha]_{\mathrm{D}}^{33}=+26.4(\mathrm{MeOH}, c=10.0)$; IR (film) $v_{\max }=2943,2861,1725$, 1461, 1425, 1284, 1202, 1155, 1114, 1079, 1032, 1002, 867, 826, 803, 744, 703, 609, $503 \mathrm{~cm}^{-1} ;{ }^{1} \mathrm{H}$ NMR (500 MHz, $\mathrm{CDCl}_{3}$ ): $\delta=$ 7.75-7.65 (m, 4 H), 7.45-7.35 (m, 6 H), 6.00 (m, 1 H), 5.75-5.65 (m, 1 H), 5.49 (dd, $J=15.8,6.6$ Hz, 1 H), 4.48-4.42 (m, 1 H), 4.40-4.33 (m, 1 H), 4.15 (br s, 1 H), 4.00 (t, $J$ = $6.6 \mathrm{~Hz}, 2 \mathrm{H}$ ), 3.89-3.82 (m, $1 \mathrm{H}), 3.75$ (dd, $J=11.0,3.7 \mathrm{~Hz}, 1 \mathrm{H}), 3.64$ (dd, $J=11.0,4.4 \mathrm{~Hz}, 1 \mathrm{H}$ ), 2.50-1.35 (m, 15 H), 1.23-0.95 (s, $18 \mathrm{H}), 0.88$ (d, $J=6.6 \mathrm{~Hz}, 3 \mathrm{H}) ;{ }^{13} \mathrm{C}$ NMR (125 MHz, $\mathrm{CD}_{2} \mathrm{Cl}_{2}$ ): $\delta=$ 
178.6, 145.6, 135.9, 134.1, 132.7, 129.9, 129.5, 128.0, 118.9, 112.2, 103.4, 79.3, 76.8, 76.1, 68.8, 66.7, 63.8, 38.9, 36.0, 34.1, 34.0, 32.3, 31.0, 30.9, 28.9, 28.3, 27.3, 27.0, 23.2, 19.4, 15.6; HR-MS [electrospray ionization (ESI)]: calcd for $\mathrm{C}_{43} \mathrm{H}_{57} \mathrm{O}_{10} \mathrm{SSiF}_{3} \mathrm{Na}^{+}\left[\mathrm{M}+\mathrm{Na}^{+}\right.$]: 873.3286, found 873.3284.

Primary TBDPS ether 83. Known lactone $\mathbf{8 1}^{4}$ (1.49 g, 7.52 mmol, 1.0 equiv) was dissolved in $\mathrm{H}_{2} \mathrm{O}$ $(1.5 \mathrm{~mL})$ and glacial acetic acid $(3.0 \mathrm{~mL})$ and then heated to $55^{\circ} \mathrm{C}$ for $2 \mathrm{~h}$. After cooling to $25^{\circ} \mathrm{C}$, the

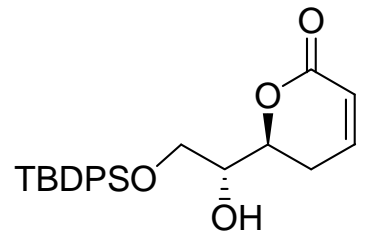

83 reaction mixture was azeotroped with toluene $(4 \times 20 \mathrm{~mL})$ to obtain the crude diol $\mathbf{8 2}$ which was carried on to the next step without further purification. Diol 82 was then dissolved in $\mathrm{CH}_{2} \mathrm{Cl}_{2}(5 \mathrm{~mL})$ and cooled to $0{ }^{\circ} \mathrm{C}$. To the solution was added $\mathrm{Et}_{3} \mathrm{~N}$ (3.14 mL, $22.6 \mathrm{mmol}, 3.0$ equiv), 4-DMAP (74 mg, $0.6 \mathrm{mmol}, 0.1$ equiv), and TBDPSCl ( $2.74 \mathrm{~mL}, 10.5 \mathrm{mmol}, 1.5$ equiv). The reaction mixture was then warmed to $25^{\circ} \mathrm{C}$ over 4 $\mathrm{h}$ and then quenched with saturated aqueous $\mathrm{NH}_{4} \mathrm{Cl}(10 \mathrm{~mL})$. The biphasic mixture was extracted with $\mathrm{CH}_{2} \mathrm{Cl}_{2}(3 \times 20 \mathrm{~mL})$ and the combined organic layers were dried $\left(\mathrm{Na}_{2} \mathrm{SO}_{4}\right)$, concentrated, and then purified by flash column chromatography (silica gel, EtOAc:hexanes 1:2) to afford primary TBDPS ether 83 (2.95 g, $7.44 \mathrm{mmol}, 99 \%$ ) as a white foam. 83: $R_{\mathrm{f}}=0.28$ (silica gel, EtOAc:hexanes 1:3); $[\alpha]_{\mathrm{D}}{ }^{33}$ $=-41.3\left(\mathrm{CHCl}_{3}, c=0.87\right)$; IR (film) $v_{\max }=3429,2931,2856,1727,1427,1387,1252,1111,1078 \mathrm{~cm}^{-1}$; ${ }^{1} \mathrm{H}$ NMR (600 MHz, $\mathrm{CDCl}_{3}$ ): $\delta=7.70-7.60$ (m, 4 H), 7.46-7.38 (m, $6 \mathrm{H}$ ), 6.91 (ddd, $J=9.8,5.7,2.6$ Hz, 1 H), 6.01 (ddd, $J$ = 9.8, 2.6, 1.0 Hz, 1 H), 4.46 (ddd, $J=11.0,6.4,4.4$ Hz, 1 H), 3.94-3.87 (m, 1 H), 3.87 (dd, $J=10.3,5.0 \mathrm{~Hz}, 1 \mathrm{H}$ ), 3.82 (dd, $J=10.3,4.6 \mathrm{~Hz}, 1 \mathrm{H}$ ), 2.55 (d, $J=5.3 \mathrm{~Hz}, 1 \mathrm{H}$ ), 2.53 (ddt, $J=$ 18.6, 11.2, $2.6 \mathrm{~Hz}, 1 \mathrm{H}$ ), 2.46 (dddd, $J=18.6,5.9,4.6,1.0 \mathrm{~Hz}, 1 \mathrm{H}), 1.07$ (s, $9 \mathrm{H}$ ); ${ }^{13} \mathrm{C}$ NMR (150 $\left.\mathrm{MHz}, \mathrm{CDCl}_{3}\right): \delta=163.5,145.4,135.5,132.7,132.6,130.1,130.0,127.9,127.8,121.1,121.0,77.3$, 72.0, 63.5, 26.8, 25.1, 19.2; HR-MS [Matrix-assisted laser desorption / ionization (MALDI)]: calcd for $\mathrm{C}_{23} \mathrm{H}_{28} \mathrm{O}_{4} \mathrm{SiNa}^{+}\left[\mathrm{M}+\mathrm{Na}^{+}\right]:$419.1649, found 419.1633.

Bis-silyl ether 84. Primary TBDPS ether 83 ( $0.65 \mathrm{~g}, 1.54 \mathrm{mmol}$, 1.0 equiv) was dissolved in $\mathrm{CH}_{2} \mathrm{Cl}_{2}(10$ $\mathrm{mL}$ ) and cooled to $0{ }^{\circ} \mathrm{C}$. To the solution was added 2,6-lutidine (0.477 mL, $4.62 \mathrm{mmol}, 3.0$ equiv), followed by the dropwise addition of TESOTf ( $0.67 \mathrm{~mL}, 3.08 \mathrm{mmol}, 2.0$ equiv). The reaction mixture 
was stirred for $1 \mathrm{~h}$, then quenched with saturated aqueous $\mathrm{NH}_{4} \mathrm{Cl}(40 \mathrm{~mL})$. The biphasic mixture was extracted with $\mathrm{CH}_{2} \mathrm{Cl}_{2}(3 \times 50 \mathrm{~mL})$ and the combined organic extracts were dried $\left(\mathrm{Na}_{2} \mathrm{SO}_{4}\right)$,

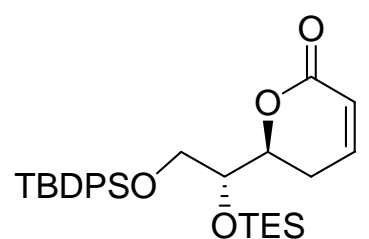

84 concentrated, and then purified by flash column chromatography (silica gel, EtOAc:hexanes 1:9) to afford bis-silyl ether 84 (740 $\mathrm{mg}, 1.36 \mathrm{mmol}, 88 \%)$ as a colorless oil. 84: $R_{\mathrm{f}}=0.57$ (silica gel, EtOAc:hexanes 1:4); $[\alpha]_{\mathrm{D}}{ }^{33}=-36.2$ $\left(\mathrm{CHCl}_{3}, c=0.69\right)$; IR (film) $v_{\max }=2941,2936,2821,1726,1431,1387,1255$, $1112 \mathrm{~cm}^{-1}$; ${ }^{1} \mathrm{H}$ NMR (600 MHz, $\mathrm{CDCl}_{3}$ ): $\delta=7.70-7.60$ (m, $\left.4 \mathrm{H}\right), 7.46-7.38$ (m, $\left.6 \mathrm{H}\right), 6.86$ (ddd, $J=9.5$, 6.2, $2.2 \mathrm{~Hz}, 1 \mathrm{H}), 6.03$ (dd, $J=9.5,2.6 \mathrm{~Hz}, 1 \mathrm{H}$ ), 4.72 (ddd, $J=8.2,4.9,2.9 \mathrm{~Hz}, 1 \mathrm{H}$ ), 3.81-3.75 (m, 1 H), 3.66 (dd, $J=10.6,4.9$ Hz, 1 H), 3.48 (dd, $J=10.6,8.2$ Hz, 1 H), 2.61 (ddt, $J=18.5,12.5,2.5$ Hz, 1 H), 2.07 (ddd, $J=18.5,6.3,4.1 \mathrm{~Hz}, 1 \mathrm{H}), 1.04$ (s, $9 \mathrm{H}), 0.86$ (t, $J=8.0 \mathrm{~Hz}, 9 \mathrm{H}), 0.53$ (q, $J=8.0 \mathrm{~Hz}, 6$ $\mathrm{H}) ;{ }^{13} \mathrm{C}$ NMR (150 MHz, $\left.\mathrm{CDCl}_{3}\right): \delta=164.5,145.7,135.5,132.7,132.6,130.1,130.0,127.9,127.8$, 121.1, 121.0, 77.3, 73.6, 72.7, 26.8, 25.4, 20.1, 19.2, 6.8; HR-MS [Matrix-assisted laser desorption / ionization (MALDI)]: calcd for $\mathrm{C}_{29} \mathrm{H}_{42} \mathrm{O}_{4} \mathrm{Si}_{2} \mathrm{Na}^{+}\left[\mathrm{M}+\mathrm{Na}^{+}\right]$: 533.2519, found 533.2534.

Methylated lactone 85. To a suspension of CuCN (192 mg, $2.15 \mathrm{mmol}, 1.5$ equiv) in $\mathrm{Et}_{2} \mathrm{O}(10 \mathrm{~mL})$ at $78{ }^{\circ} \mathrm{C}$ was added $\mathrm{MeLi}\left(3.4 \mathrm{~mL}, 1.5 \mathrm{M}\right.$ in $\mathrm{Et}_{2} \mathrm{O}, 4.3 \mathrm{mmol}, 3.0$ equiv). The mixture was allowed to warm

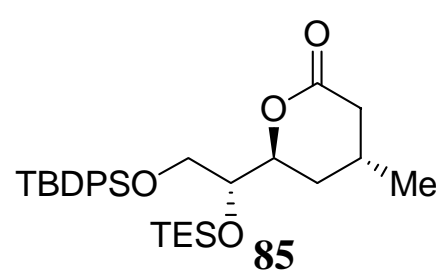
to $0{ }^{\circ} \mathrm{C}$ over $1 \mathrm{~h}$ and was then re-cooled to $-78{ }^{\circ} \mathrm{C}$. A pre-cooled $\left(-78{ }^{\circ} \mathrm{C}\right)$ solution of bis-silyl ether 84 (0.73 g, 1.43 mmol, 1.0 equiv) in $\mathrm{Et}_{2} \mathrm{O}(10 \mathrm{~mL})$ was then added dropwise. After 20 min the mixture was quenched with a 10:1 mixture of saturated aqueous $\mathrm{NH}_{4} \mathrm{Cl}: 25 \% \mathrm{NH}_{4} \mathrm{OH}(50 \mathrm{~mL})$, and the resulting biphasic mixture was stirred for an additional $2.5 \mathrm{~h}$. The mixture was then extracted with $\mathrm{Et}_{2} \mathrm{O}$ $(3 \times 30 \mathrm{~mL})$ and the combined organic extracts were dried $\left(\mathrm{Na}_{2} \mathrm{SO}_{4}\right)$, concentrated, and then purified by flash column chromatography (silica gel, EtOAc:hexanes 1:1) to give a single isomer of methylated lactone 85 (0.73 g, $1.38 \mathrm{mmol}, 97 \%)$ as a colorless oil. 85: $R_{\mathrm{f}}=0.60$ (silica gel, EtOAc:hexanes 1:4); $[\alpha]_{\mathrm{D}}{ }^{25}=-3.4\left(\mathrm{CHCl}_{3}, c=5.6\right) ;$ IR (film) $v_{\max }=2955,1744,1463,1235,1082,1008 \mathrm{~cm}^{-1} ;{ }^{1} \mathrm{H}$ NMR (500 MHz, $\left.\mathrm{CDCl}_{3}\right): \delta=7.70-7.60$ (m, $\left.4 \mathrm{H}\right)$, 7.47-7.38 (m, $\left.6 \mathrm{H}\right), 4.76-4.70(\mathrm{~m}, 1 \mathrm{H}), 4.08-4.01$ (m, 1 H), 3.63 (dd, $J=10.3,5.2 \mathrm{~Hz}, 1 \mathrm{H}), 3.49$ (dd, $J=10.3,8.6 \mathrm{~Hz}, 1 \mathrm{H}), 2.52$ (dd, $J=16.3$, $5.3 \mathrm{~Hz}, 1 \mathrm{H}$ ), 
2.29-2.20 (m, 1 H), 2.18 (dd, $J=16.3,7.3$ Hz, 1 H), 2.05 (ddd, $J$ = 14.5, 9.1, 5.7 Hz, 1 H), 1.45 (dt, $J=$ 14.5, $5.5 \mathrm{~Hz}, 1 \mathrm{H}), 1.07$ (d, $J=6.5 \mathrm{~Hz}, 3 \mathrm{H}), 1.04$ (s, $9 \mathrm{H}$ ), 0.85 (t, $J=8.0 \mathrm{~Hz}, 9 \mathrm{H}$ ), 0.49 (q, $J=8.0 \mathrm{~Hz}$, $6 \mathrm{H}$ ); ${ }^{13} \mathrm{C}$ NMR (150 MHz, $\mathrm{CDCl}_{3}$ ): $\delta=172.1,135.5,135.1,133.1,132.9,129.87,129.82,127.78$, 127.76, 77.3, 73.3, 64.3, 37.7, 27.5, 26.8, 24.0, 20.7, 19.1, 6.7, 4.7; HR-MS [Matrix-assisted laser desorption / ionization (MALDI)]: calcd for $\mathrm{C}_{30} \mathrm{H}_{46} \mathrm{O}_{4} \mathrm{Si}_{2} \mathrm{Na}^{+}\left[\mathrm{M}+\mathrm{Na}^{+}\right]$: 549.2827, found 549.2813.

Bicycle 87. To a solution of (benzyloxymethyl)-tri-n-butylstannane ( $0.284 \mathrm{~g}, 0.68 \mathrm{mmol}, 1.8$ equiv) in THF ( $4 \mathrm{~mL})$ at $-78{ }^{\circ} \mathrm{C}$ was added $n$-BuLi $(0.46 \mathrm{~mL}, 1.6 \mathrm{M}$ in hexanes, $0.65 \mathrm{mmol}, 1.7$ equiv). After 15 OBn $\mathrm{min}$ a solution of methylated lactone 85 (200 mg, $0.38 \mathrm{mmol}, 1.0$ equiv) in
TBDP $(3 \mathrm{~mL})$ was added dropwise. The reaction mixture was allowed to warm $\mathrm{NH}_{4} \mathrm{Cl}(30 \mathrm{~mL})$. The biphasic mixture was extracted with EtOAc $(3 \times 20 \mathrm{~mL})$ and the combined organic extracts were dried $\left(\mathrm{Na}_{2} \mathrm{SO}_{4}\right)$ and concentrated to afford crude lactol 86, which was used in the next step without any further purification. To a solution of crude lactol 86 in $\mathrm{CH}_{2} \mathrm{Cl}_{2}(1.5 \mathrm{~mL})$ at $25{ }^{\circ} \mathrm{C}$ was added p-TsOH (9 mg, $0.05 \mathrm{mmol}, 0.1$ equiv). The reaction mixture was allowed to stir for $1 \mathrm{~h}$ and then quenched with saturated aqueous $\mathrm{NaHCO}_{3}(10 \mathrm{~mL})$. The biphasic mixture was extracted with $\mathrm{Et}_{2} \mathrm{O}(3 \times$ $10 \mathrm{~mL})$ and the combined organic extracts were dried $\left(\mathrm{Na}_{2} \mathrm{SO}_{4}\right)$, concentrated, and then purified by flash column chromatography (silica gel, EtOAc:hexanes 1:19) to afford a single isomer of bicycle 87 (87 mg, $0.17 \mathrm{mmol}, 45 \%$ over two steps) as a colorless oil. 87: $R_{\mathrm{f}}=0.59$ (silica gel, EtOAc:hexanes 1:4); $[\alpha]_{\mathrm{D}}{ }^{25}$ $=-1.8\left(\mathrm{CHCl}_{3}, c=3.5\right)$; IR (film) $v_{\max }=2948,2932,2857,1517,1448,1223,1112,1058 \mathrm{~cm}^{-1} ;{ }^{1} \mathrm{H}$ NMR (500 MHz, $\left.\mathrm{CDCl}_{3}\right): \delta=7.70-7.60(\mathrm{~m}, 4 \mathrm{H}), 7.45-7.26$ (m, $\left.6 \mathrm{H}\right), 4.65$ (d, $\left.J=12.2 \mathrm{~Hz}, 1 \mathrm{H}\right), 4.60$ (d, $J=12.2 \mathrm{~Hz}, 1 \mathrm{H}$ ), 4.51-4.48 (m, 1 H), 4.26-4.20 (m, 1 H), 4.05 (dd, $J=10.6,5.8$ Hz, 1 H), 3.82 (dd, $J=10.6,8.3 \mathrm{~Hz}, 1 \mathrm{H}), 3.55$ (d, $J=12.0 \mathrm{~Hz}, 1 \mathrm{H}), 3.51$ (d, $J=12.0 \mathrm{~Hz}, 1 \mathrm{H}), 1.98-1.91$ (m, $2 \mathrm{H}), 1.76$ (dd, $J=13.1,5.7 \mathrm{~Hz}, 1 \mathrm{H}), 1.65-1.60$ (m, 2 H), 1.48-1.42 (m, 4 H), 1.34 (dd, $J=13.2,11.0 \mathrm{~Hz}, 1 \mathrm{H}$ ), 1.06 (s, $9 \mathrm{H}$ ), 0.84 (d, $J=6.5 \mathrm{~Hz}, 3 \mathrm{H}) ;{ }^{13} \mathrm{C}$ NMR $\left(125 \mathrm{MHz}, \mathrm{CDCl}_{3}\right): \delta=138.1,135.61,135.59,135.58$, 135.56, 133.4, 133.1, 129.8, 128.3, 127.7, 127.63, 127.5, 107.4, 79.7, 76.3, 73.6, 73.2, 61.8, 39.3, 33.0, 
26.8, 24.0, 21.9, 19.2; HR-MS [Matrix-assisted laser desorption / ionization (MALDI)]: calcd for $\mathrm{C}_{32} \mathrm{H}_{40} \mathrm{O}_{4} \mathrm{SiNa}^{+}\left[\mathrm{M}+\mathrm{Na}^{+}\right]:$539.2594, found 539.2609.

Primary Alcohol 88. To a solution of bicycle 87 (56.0 mg, $0.108 \mathrm{mmol}, 1.0$ equiv) in THF (1.0 mL) at $25{ }^{\circ} \mathrm{C}$ was added TBAF $(0.163 \mathrm{~mL}, 0.163 \mathrm{mmol}, 1.5$ equiv). The reaction mixture was stirred for $30 \mathrm{~min}$ $-\mathrm{OBn}$ and then quenched with saturated aqueous $\mathrm{NH}_{4} \mathrm{Cl}(5 \mathrm{~mL})$. The biphasic mixture was extracted with EtOAc $(3 \times 5 \mathrm{~mL})$ and the combined organic extracts were dried $\left(\mathrm{Na}_{2} \mathrm{SO}_{4}\right)$, concentrated, and then purified by flash column chromatography (silica gel, EtOAc:hexanes 1:1) to afford primary alcohol 88 (28.1 mg, $0.95 \mathrm{mmol}, 93 \%)$ as a colorless oil. 88: $R_{\mathrm{f}}=0.32$ (silica gel, EtOAc:hexanes 1:1); $[\alpha]_{\mathrm{D}}{ }^{25}=-53.3\left(\mathrm{CHCl}_{3}, c=1.8\right)$; IR (film) $v_{\max }=$ 3440, 2954, 1455, 1108, 1026, $744 \mathrm{~cm}^{-1} ;{ }^{1} \mathrm{H}$ NMR (500 MHz, $\mathrm{CDCl}_{3}$ ): $\delta=7.37-7.26$ (m, $\left.5 \mathrm{H}\right), 4.65$ (d, $J=12.0 \mathrm{~Hz}, 1 \mathrm{H}), 4.61$ (d, $J=12.0 \mathrm{~Hz}, 1 \mathrm{H}), 4.38-4.31$ (m, $1 \mathrm{H}), 4.04$ (q, $J=5.9 \mathrm{~Hz}, 1 \mathrm{H}), 3.88$ (dd, $J=$ 11.0, $6.0 \mathrm{~Hz}, 1 \mathrm{H}$ ), 3.71 (dd, $J=11.0,6.8 \mathrm{~Hz}, 1 \mathrm{H}), 3.53$ (d, $J=12.0 \mathrm{~Hz}, 1 \mathrm{H}), 3.50$ (d, $J=12.0 \mathrm{~Hz}, 1$ H), 2.15-2.11 (m, 1 H), 1.80 (t, $J=6.3 \mathrm{~Hz}, 1 \mathrm{H}), 1.77$ (t, $J=6.9 \mathrm{~Hz}, 1 \mathrm{H}), 1.40-1.35$ (m, $1 \mathrm{H}), 1.31$ (dd, $J=13.2,11.0 \mathrm{~Hz}, 1 \mathrm{H}), 0.91$ (d, $J=6.5 \mathrm{~Hz}, 3 \mathrm{H}) ;{ }^{13} \mathrm{C}$ NMR $\left(125 \mathrm{MHz}, \mathrm{CDCl}_{3}\right): \delta=139.7,129.0,128.3$, 128.2, 108.1, 81.6, 76.6, 73.8, 67.8, 60.7, 40.1, 34.0, 24.9, 22.4; HR-MS [Matrix-assisted laser desorption / ionization (MALDI)]: calcd for $\mathrm{C}_{16} \mathrm{H}_{22} \mathrm{O}_{4} \mathrm{Na}^{+}\left[\mathrm{M}+\mathrm{Na}^{+}\right]$: 301.1416, found 301.1419.

Weinreb Amide 90. To a solution of N,O-dimethylamine hydrochloride (1.93 g, 1.98 mmol, 5.0 equiv) in THF (12 mL) at $0{ }^{\circ} \mathrm{C}$ was slowly added $\mathrm{AlMe}_{3}(9.9 \mathrm{~mL}, 2.0 \mathrm{M}$ in toluene, $1.98 \mathrm{mmol}, 5.0$ equiv) $\underbrace{\substack{N^{-} \\ \mathbf{9 0}}}_{\substack{\overline{\bar{M}} \\ \mathrm{Me}}} \begin{aligned} & \text { THF }(12 \mathrm{~mL}) \text {. The reaction mixture was allowed to stir for } 4 \mathrm{~h} \text { at } 0{ }^{\circ} \mathrm{C} \text {, at which } \\ & \text { time hexanes }(30 \mathrm{~mL}), \mathrm{CH}_{2} \mathrm{Cl}_{2}(10 \mathrm{~mL}) \text {, and tartaric acid }\left(1 \mathrm{M} \text { in } \mathrm{H}_{2} \mathrm{O}, 25 \mathrm{~mL}\right)\end{aligned}$ were added. Vigorous stirring was continued for $1 \mathrm{~h}$ and then the biphasic mixture was extracted with $\mathrm{CH}_{2} \mathrm{Cl}_{2}(3 \times 40 \mathrm{~mL})$. The combined organic extracts were dried $\left(\mathrm{Na}_{2} \mathrm{SO}_{4}\right)$, concentrated, and then purified by flash column chromatography (silica gel, EtOAc:hexanes 1:1) to afford Weinreb amide 90 (0.718 g, $3.80 \mathrm{mmol}, 96 \%)$ as a colorless oil. 90: $R_{\mathrm{f}}=0.72$ (silica gel, EtOAc:hexanes 1:1); IR (film) 
$v_{\max }=3420,2930,2887,1729,1452,1234,1119 \mathrm{~cm}^{-1} ;{ }^{1} \mathrm{H}$ NMR $\left(500 \mathrm{MHz}, \mathrm{CDCl}_{3}\right): \delta=3.69(\mathrm{~s}, 3 \mathrm{H})$, 3.44-3.35 (m, 2 H), 3.16 (s, 3 H), 3.01-2.90 (m, 1 H), 2.26-2.20 (m, 1 H), 1.92-1.85 (m, 1 H), 1.56-1.48 (m, $1 \mathrm{H}), 1.12-1.08$ (m, $1 \mathrm{H}), 1.11$ (d, $J=6.5 \mathrm{~Hz}, 3 \mathrm{H}), 0.91$ (d, $J=6.5 \mathrm{~Hz}, 3 \mathrm{H}) ;{ }^{13} \mathrm{C}$ NMR $\left(125 \mathrm{MHz}, \mathrm{CDCl}_{3}\right): \delta=173.7,67.2,61.4,60.5,36.9,34.4,33.4,18.6,17.4$; HR-MS [Matrix-assisted laser desorption / ionization (MALDI)]: calcd for $\mathrm{C}_{9} \mathrm{H}_{19} \mathrm{NO}_{3} \mathrm{Na}^{+}\left[\mathrm{M}+\mathrm{Na}^{+}\right]$: 212.1257, found 212.1257.

Tosylate 91. To a solution of Weinreb amide 90 (0.718 g, $3.80 \mathrm{mmol}, 1.0$ equiv) in $\mathrm{CH}_{2} \mathrm{Cl}_{2}(20 \mathrm{~mL})$ at $25{ }^{\circ} \mathrm{C}$ was added $\mathrm{Et}_{3} \mathrm{~N}$ (3.0 mL, $19.0 \mathrm{mmol}, 5.5$ equiv), followed by $\mathrm{p}$-TsCl (1.13 g, $5.70 \mathrm{mmol}, 1.5$ OTs $\quad \mathrm{O}$ equiv). The reaction mixture was allowed to stir at $25^{\circ} \mathrm{C}$ for $18 \mathrm{~h}$ and was then

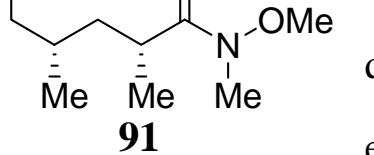
quenched with saturated aqueous $\mathrm{NaHCO}_{3}(30 \mathrm{~mL})$. The biphasic mixture was extracted with $\mathrm{CH}_{2} \mathrm{Cl}_{2}(3 \times 30 \mathrm{~mL})$ and the combined organic extracts were dried ( $\left.\mathrm{Na}_{2} \mathrm{SO}_{4}\right)$, concentrated, and then purified by flash column chromatography (silica gel, EtOAc:hexanes 1:1) to afford tosylate 91 (1.25 g, $3.70 \mathrm{mmol}, 96 \%$ ) as a colorless oil. 91: $R_{\mathrm{f}}=0.50$ (silica gel, ethyl acetate: hexanes 1:3); IR (film) $v_{\max }=2931,2879,1729,1654,1412 \mathrm{~cm}^{-1} ;{ }^{1} \mathrm{H} \mathrm{NMR} \quad(500 \mathrm{MHz}$, $\left.\mathrm{CDCl}_{3}\right): \delta=7.75$ (d, $\left.J=8.0 \mathrm{~Hz}, 2 \mathrm{H}\right), 7.32$ (d, $\left.J=8.0 \mathrm{~Hz}, 2 \mathrm{H}\right), 3.85$ (dd, $\left.J=9.5,5.0 \mathrm{~Hz}, 1 \mathrm{H}\right), 3.76$ (dd, $J=9.5,6.5 \mathrm{~Hz}, 1 \mathrm{H}), 3.66$ (s, $3 \mathrm{H}), 3.13$ (s, $3 \mathrm{H}), 2.96$ (br s, $1 \mathrm{H}), 2.42$ (s, $3 \mathrm{H}), 1.77-1.70$ (m, $1 \mathrm{H})$, 1.71-1.64 (m, $1 \mathrm{H}), 1.17-1.11$ (m, $1 \mathrm{H}), 1.06$ (d, $J=6.5 \mathrm{~Hz}, 3 \mathrm{H}), 0.89$ (d, $J=6.5 \mathrm{~Hz}, 3 \mathrm{H}) ;{ }^{13} \mathrm{C}$ NMR (125 $\mathrm{MHz}_{\mathrm{CDCl}}$ ): $\delta=174.2,144.6,132.8,129.7,127.8,75.0,61.5,36.8,32.3,32.0,30.7,21.5,18.0$, 16.7; HR-MS [Matrix-assisted laser desorption / ionization (MALDI)]: calcd for $\mathrm{C}_{16} \mathrm{H}_{25} \mathrm{NO}_{5} \mathrm{SH}^{+}[\mathrm{M}+$ $\left.\mathrm{H}^{+}\right]$: 344.1526, found 344.1526.

Azide 92. To a solution of tosylate 91 (0.508 g, $1.48 \mathrm{mmol}, 1.0$ equiv) in DMF (8 mL) at $25^{\circ} \mathrm{C}$ was added $\mathrm{NaN}_{3}(0.193 \mathrm{~g}, 2.96 \mathrm{mmol}, 2.0$ equiv). The reaction mixture was allowed to stir for $96 \mathrm{~h}$, at which

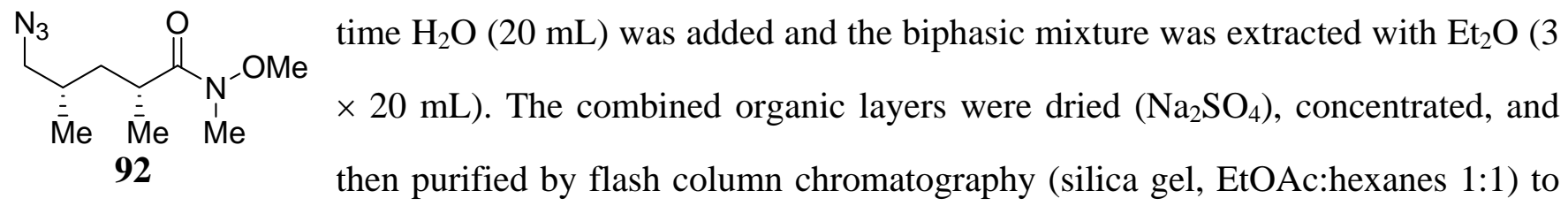
afford azide 92 (0.304 g, 1.42 mmol, 96\%) as a colorless oil. 92: $R_{\mathrm{f}}=0.66$ (silica gel, EtOAc:hexanes 
1:1); IR (film) $v_{\max }=2934,2865,1728,1612,1454,1211 \mathrm{~cm}^{-1} ;{ }^{1} \mathrm{H}$ NMR $\left(500 \mathrm{MHz}, \mathrm{CDCl}_{3}\right): \delta=3.69$ (s, 3 H), 3.24 (dd, $J=12.0,5.0$ Hz, 1 H), 3.17 (s, 3 H), 3.08 (dd, $J=12.0,7.0 \mathrm{~Hz}, 1 \mathrm{H}$ ), 3.03-2.97 (m, 1 H), 1.80 (ddd, $J=14.0,9.0,5.0 \mathrm{~Hz}, 1 \mathrm{H}), 1.70-1.63$ (m, $1 \mathrm{H}), 1.16$ (ddd, $J=14.0,8.0,5.0 \mathrm{~Hz}, 1 \mathrm{H}$ ), $1.13(\mathrm{~d}, J=6.5 \mathrm{~Hz}, 3 \mathrm{H}), 0.96$ (d, $J=6.5 \mathrm{~Hz}, 3 \mathrm{H}) ;{ }^{13} \mathrm{C} \mathrm{NMR}\left(125 \mathrm{MHz}, \mathrm{CDCl}_{3}\right): \delta=173.5,64.0,61.4$, 57.8, 38.3, 32.6, 31.6, 18.2, 17.8; HR-MS [Matrix-assisted laser desorption / ionization (MALDI)]: calcd for $\mathrm{C}_{9} \mathrm{H}_{18} \mathrm{~N}_{4} \mathrm{O}_{2} \mathrm{Na}^{+}\left[\mathrm{M}+\mathrm{Na}^{+}\right]$: 237.1322, found 237.1327.

Methyl Ketone 23. To a solution of azide 92 (1.19 g, $5.61 \mathrm{mmol}, 1.0$ equiv) in THF (32 mL) at $-78{ }^{\circ} \mathrm{C}$ was added MeLi (3.7 mL, 1.6 $\mathrm{M}$ in $\mathrm{Et}_{2} \mathrm{O}, 5.89 \mathrm{mmol}, 1.05$ equiv). The reaction mixture was stirred at -<smiles>CC(=O)[C@H](C)C[C@@H](C)CN</smiles$78{ }^{\circ} \mathrm{C}$ for $1 \mathrm{~h}$ and then quenched with a $1 \mathrm{M}$ solution of $\mathrm{NaH}_{2} \mathrm{PO}_{4}(10 \mathrm{~mL})$. The biphasic mixture was extracted with EtOAc $(3 \times 30 \mathrm{~mL})$ and the combined organic extracts were dried $\left(\mathrm{Na}_{2} \mathrm{SO}_{4}\right)$, concentrated, and then purified by flash column chromatography (silica gel, EtOAc:hexanes 1:3) to afford methyl ketone 23 (0.777g, 4.60 mmol, 82\%) as a colorless oil. 23: $R_{\mathrm{f}}=0.50$ (silica gel, EtOAc:hexanes 1:4); IR (film) $v_{\max }=2929,2856,1729$, 1412, $1112 \mathrm{~cm}^{-1}$; ${ }^{1} \mathrm{H}$ NMR (500 MHz, $\mathrm{CDCl}_{3}$ ): $\delta=3.20$ (dd, $J=12.0,6.0 \mathrm{~Hz}, 1 \mathrm{H}$ ), 3.13 (dd, $J=12.0$, 6.5 Hz, 1 H), 2.64-2.57 (m, 1 H), 2.14 (s, 3 H), 1.80 (ddd, J = 14.0, 8.5, 5.5 Hz, 1 H), 1.71-1.64 (m, 1 H), 1.15-1.09 (m, $1 \mathrm{H}), 1.10$ (d, $J=6.5 \mathrm{~Hz}, 3 \mathrm{H}), 0.95$ (d, $J=6.5 \mathrm{~Hz}, 3 \mathrm{H}) ;{ }^{13} \mathrm{C}$ NMR $(125 \mathrm{MHz}$, $\left.\mathrm{CDCl}_{3}\right): \delta=212.2,57.6,44.7,37.2,31.5,27.9,17.8,17.3$; HR-MS [Matrix-assisted laser desorption / ionization (MALDI)]: calcd for $\mathrm{C}_{8} \mathrm{H}_{15} \mathrm{~N}_{3} \mathrm{ONa}^{+}\left[\mathrm{M}+\mathrm{Na}^{+}\right]$: 192.1107, found 192.1110.

Aldol Product 22. To a solution of methyl ketone 23 (8.5 mg, $0.050 \mathrm{mmol}, 1.0$ equiv) in THF (2 mL) at $-78{ }^{\circ} \mathrm{C}$ was added lithium diisopropylamide $(0.75 \mathrm{~mL}, 1.0 \mathrm{M}$ in THF, $0.075 \mathrm{mmol}, 1.5$ equiv) followed<smiles>C[C@H](CN)C[C@H](C)C(=O)C[C@@H](O)[C@H]1O[C@]2(COc3ccccc3)C[C@H](C)C[C@H]1O2</smiles>
by TESCl $(0.25 \mathrm{~mL}, 0.15 \mathrm{mmol}, 3.0$ equiv). The reaction mixture was stirred for $30 \mathrm{~min}$ at $-78{ }^{\circ} \mathrm{C}$, and then quenched with saturated aqueous $\mathrm{NaHCO}_{3}(3 \mathrm{~mL})$. The biphasic mixture was extracted with EtOAc $(3 \times 3$ $\mathrm{mL})$ and the combined organic extracts were dried $\left(\mathrm{Na}_{2} \mathrm{SO}_{4}\right)$ and concentrated, affording silyl enol ether 93 (11.0 mg, $0.043 \mathrm{mmol}$, 86\%), which was used directly in the 
aldol reaction without further purification. To a solution of aldehyde 24 (12.0 mg, $0.043 \mathrm{mmol}, 1.0$ equiv) in $\mathrm{CH}_{2} \mathrm{Cl}_{2}(1.0 \mathrm{~mL})$ at $-78{ }^{\circ} \mathrm{C}$ was added $\mathrm{SnCl}_{4}(0.043 \mathrm{~mL}, 1.0 \mathrm{M}$ in toluene, $0.043 \mathrm{mmol}, 1.0$ equiv). The solution was stirred for $5 \mathrm{~min}$ and then a solution of silyl enol ether 93 (11.0 mg, 0.043 mmol, 1.0 equiv) in $\mathrm{CH}_{2} \mathrm{Cl}_{2}(1.0 \mathrm{~mL})$ was added and the resulting mixture was allowed to stir at $-78{ }^{\circ} \mathrm{C}$ for $1 \mathrm{~h}$. The reaction mixture was then quenched with saturated aqueous $\mathrm{NaHCO}_{3}(5 \mathrm{~mL})$ and extracted with $\mathrm{CH}_{2} \mathrm{Cl}_{2}(3 \times 5 \mathrm{~mL})$. The combined organic extracts were dried $\left(\mathrm{Na}_{2} \mathrm{SO}_{4}\right)$, concentrated, and then purified by flash column chromatography (silica gel, EtOAc:hexanes 1:3) to afford a single isomer of aldol product 22 (9.6 mg, $0.022 \mathrm{mmol}, 50 \%$ ) as a colorless oil. 22: $R_{\mathrm{f}}=0.19$ (silica gel, EtOAc:hexanes $1: 4) ;[\alpha]_{\mathrm{D}}^{25}=-13.8\left(c=0.10, \mathrm{CHCl}_{3}\right)$; IR (film) $v_{\max }=2923,2097,1702,1455,1102 \mathrm{~cm}^{-1} ;{ }^{1} \mathrm{H} \mathrm{NMR}$ (600 MHz, CDCl $): \delta=7.37-7.26$ (m, 5 H), 4.65 (d, $J=12.0 \mathrm{~Hz}, 1 \mathrm{H}$ ), 4.61 (d, $J=12.0 \mathrm{~Hz}, 1 \mathrm{H}$ ), 4.52 (t, $J=4.0 \mathrm{~Hz}, 1 \mathrm{H}$ ), 3.81 (dd, $J=$ 9.9, $4.0 \mathrm{~Hz}, 1 \mathrm{H}$ ), 3.50 (d, $J=12.0 \mathrm{~Hz}, 1 \mathrm{H}$ ), 3.48 (d, $J=12.0 \mathrm{~Hz}, 1$ H), 3.37 (d, $J=4.0 \mathrm{~Hz}, 1 \mathrm{H}$ ), 3.18 (dd, $J=12.0,5.7 \mathrm{~Hz}, 1 \mathrm{H}$ ), 3.14 (dd, $J=12.0,6.2 \mathrm{~Hz}, 1 \mathrm{H}$ ), 3.07 (dd, $J=18.4,2.4 \mathrm{~Hz}, 1 \mathrm{H}), 2.70-2.62$ (m, 1 H), 2.62 (dd, $J=13.6,6.8 \mathrm{~Hz}, 1 \mathrm{H}), 2.10-2.03$ (m, 1 H), 1.93 (dd, $J=14.0,5.7 \mathrm{~Hz}, 1 \mathrm{H}), 1.84$ (ddd, $J=14.0,8.8,6.1 \mathrm{~Hz}, 1 \mathrm{H}$ ), 1.80 (dd, $J=13.6,6.1 \mathrm{~Hz}, 1 \mathrm{H}$ ), 1.551.50 (m, 2 H), 1.38 (dd, $J=13.6,11.4 \mathrm{~Hz}, 1 \mathrm{H}$ ), 1.14 (ddd, $J=13.6,8.3,6.1 \mathrm{~Hz}, 1 \mathrm{H}$ ), 1.13 (d, $J=7.0$ $\mathrm{Hz}, 3 \mathrm{H}), 0.96$ (d, $J=6.6 \mathrm{~Hz}, 3 \mathrm{H}), 0.94(\mathrm{~d}, J=6.5 \mathrm{~Hz}, 3 \mathrm{H}) ;{ }^{13} \mathrm{C}$ NMR $\left(150 \mathrm{MHz}, \mathrm{CDCl}_{3}\right): \delta=216.4$, 138.0, 128.4, 127.8, 127.6, 107.7, 81.0, 76.5, 73.7, 73.3, 66.5, 57.5, 45.5, 44.5, 39.3, 37.2, 32.9, 31.4, 24.2, 22.0, 17.9, 17.2; HR-MS [Matrix-assisted laser desorption / ionization (MALDI)]: calcd for $\mathrm{C}_{24} \mathrm{H}_{35} \mathrm{~N}_{3} \mathrm{O}_{5} \mathrm{Na}^{+}\left[\mathrm{M}+\mathrm{Na}^{+}\right]$: 468.2469, found 468.2486.

TBDPS Ether 106. To a solution of known diol $\mathbf{1 0 5}^{6}$ (26.9 g, $112 \mathrm{mmol}, 1.0$ equiv) in THF (350 mL) at $-78{ }^{\circ} \mathrm{C}$ was added $n$-BuLi (76.9 mL, 1.6 M in THF, $123 \mathrm{mmol}$, 1.1 equiv). The solution was allowed to OTBDPS
added to the reaction mixture which was allowed to stir at $25{ }^{\circ} \mathrm{C}$ for $18 \mathrm{~h}$. The
$\mathbf{1 0 6}$ dried $\left(\mathrm{Na}_{2} \mathrm{SO}_{4}\right)$, concentrated, and then purified by flash column chromatography (silica gel, 
EtOAc:hexanes 1:1) to afford TBDPS ether 106 (46.6 g, $97.4 \mathrm{mmol}, 87 \%)$ as a colorless oil. 106: $R_{\mathrm{f}}=$ 0.20 (silica gel, EtOAc:hexanes 1:4); IR (film) $v_{\max }=3454,3060,2931,2849,1614,1429,1109 \mathrm{~cm}^{-1}$; ${ }^{1} \mathrm{H}$ NMR (500 MHz, $\mathrm{CDCl}_{3}$ ): $\delta=7.76-7.69$ (m, $4 \mathrm{H}$ ), 7.49-7.38 (m, $\left.8 \mathrm{H}\right), 6.94-6.90$ (m, $2 \mathrm{H}$ ), 5.98 (s, $1 \mathrm{H}), 4.36-4.32$ (m, $1 \mathrm{H}), 4.21-4.16$ (m, $1 \mathrm{H}), 3.99-3.82$ (m, $3 \mathrm{H}), 3.82$ (s, $3 \mathrm{H}), 3.81-3.71(\mathrm{~m}, 1 \mathrm{H})$, 2.32-2.22 (m, $1 \mathrm{H}), 1.13$ (s, $9 \mathrm{H}) ;{ }^{13} \mathrm{C}$ NMR (125 MHz, $\mathrm{CDCl}_{3}$ ): $\delta=160.5,135.6,135.6,132.9,129.9$, 129.8, 129.6, 129.4, 128.1, 127.8, 127.7, 113.8, 104.0, 79.8, 78.6, 64.1, 62.9, 55.3, 26.8, 19.2; HR-MS [Matrix-assisted laser desorption / ionization (MALDI)]: calcd for $\mathrm{C}_{28} \mathrm{H}_{34} \mathrm{O}_{5} \mathrm{SiNa}^{+}\left[\mathrm{M}+\mathrm{Na}^{+}\right.$]: 501.2068, found 501.2059.

Diol 107. $\mathrm{BH}_{3} \cdot \mathrm{THF}$ (664 mL, $1.0 \mathrm{M}$ in THF, $664 \mathrm{mmol}, 4.0$ equiv) was added to a solution of TBDPS ether 106 (79.4 g, $166 \mathrm{mmol}, 1.0$ equiv) in THF $(125 \mathrm{~mL})$ at $0{ }^{\circ} \mathrm{C}$ over $20 \mathrm{~min}$. The reaction was then TBDPSO $\quad \mathrm{OH} \quad$ heated to reflux for $2.5 \mathrm{~h}$. The solvent was removed, the residue was dissolved in $\mathrm{CH}_{2} \mathrm{Cl}_{2}(400 \mathrm{~mL})$, and the mixture was quenched by the slow addition of water ŌPMB 107 $(600 \mathrm{~mL})$ at $0{ }^{\circ} \mathrm{C}$. The $\mathrm{pH}$ was adjusted to 8.5 by the addition of $1 \mathrm{M} \mathrm{KOH}$, and the biphasic mixture was extracted with $\mathrm{CH}_{2} \mathrm{Cl}_{2}(3 \times 200 \mathrm{~mL})$ and EtOAc $(3 \times$ $150 \mathrm{~mL})$. The combined organic layers were dried $\left(\mathrm{Na}_{2} \mathrm{SO}_{4}\right)$, concentrated, and then purified by flash column chromatography (silica gel, EtOAc:hexanes 2:1) to provide diol 107 (60.8 g, $126 \mathrm{mmol}$, 76\%) as a colorless oil. 107: $R_{\mathrm{f}}=0.29$ (silica gel, EtOAc:hexanes 1:1); $[\alpha]_{\mathrm{D}}{ }^{25}=-33.2\left(c=0.90, \mathrm{CHCl}_{3}\right)$; IR (film) $v_{\max }=3421,3060,2933,2860,1612,1428,1110 \mathrm{~cm}^{-1} ;{ }^{1} \mathrm{H} \mathrm{NMR} \quad\left(500 \mathrm{MHz}, \mathrm{CDCl}_{3}\right): \delta=$ 7.73-7.64 (m, 4 H), 7.49-7.36 (m, 6 H), 7.20-7.14 (m, 2 H), 6.90-6.80 (m, 2 H), 4.57 (d, J = 11.2 Hz, 1 H), 4.33 (d, $J=11.2 \mathrm{~Hz}, 1 \mathrm{H}), 3.80$ (s, $3 \mathrm{H}), 3.89-3.78$ (m, $3 \mathrm{H}), 3.67$ (d, $J=5.0 \mathrm{~Hz}, 2 \mathrm{H}), 3.53$ (q, $J=$ $5.0 \mathrm{~Hz}, 1 \mathrm{H}), 2.88$ (d, $J=5.5 \mathrm{~Hz}, 1 \mathrm{H}), 2.54$ (t, $J=6.2 \mathrm{~Hz}, 1 \mathrm{H}), 1.08$ (s, $9 \mathrm{H}) ;{ }^{13} \mathrm{C} \mathrm{NMR}(125 \mathrm{MHz}$, $\left.\mathrm{CDCl}_{3}\right): \delta=159.4,135.6,135.6,133.0,132.8,129.9,129.9,129.6,129.6,127.8,127.8,113.9,79.1$, 72.3, 71.7, 63.8, 63.0, 55.3, 26.8, 19.1; HR-MS [Matrix-assisted laser desorption / ionization (MALDI)]: calcd for $\mathrm{C}_{28} \mathrm{H}_{36} \mathrm{O}_{5} \mathrm{SiNa}^{+}\left[\mathrm{M}+\mathrm{Na}^{+}\right]$: 503.2224, found 503.2214. 
Aldehyde 108. To a solution of diol 107 (60.6 g, $126 \mathrm{mmol}, 1.0$ equiv) in THF (960 mL) and water (640 $\mathrm{mL}$ ) at $0{ }^{\circ} \mathrm{C}$ was added $\mathrm{NaIO}_{4}(108 \mathrm{~g}, 505 \mathrm{mmol}, 4.0$ equiv). The reaction mixture was allowed to warm TBDPSO O to $25^{\circ} \mathrm{C}$ over $4 \mathrm{~h}$, and was then quenched by the addition of saturated aqueous $\mathrm{Na}_{2} \mathrm{~S}_{2} \mathrm{O}_{3}(1.0 \mathrm{~L})$. The biphasic mixture was extracted with $\mathrm{CH}_{2} \mathrm{Cl}_{2}(4 \times 300 \mathrm{~mL})$ and ÖPMB 108 ethyl acetate $(1 \times 150 \mathrm{~mL})$ and the combined organic layers were dried $\left(\mathrm{Na}_{2} \mathrm{SO}_{4}\right)$, concentrated, and then purfied by flash column chromatography (silica gel, EtOAc:hexanes 1:1) to provide aldehyde 108 (49.6 g, $111 \mathrm{mmol}, 88 \%$ ) as a colorless oil. 108: $R_{\mathrm{f}}=0.74$ (silica gel, EtOAc:hexanes 1:1); $[\alpha]_{\mathrm{D}}^{25}=+6.7\left(\mathrm{c}=0.89, \mathrm{CHCl}_{3}\right)$; IR (film) $v_{\max }=3060,2931,2861,1735,1612$, 1249, $1110 \mathrm{~cm}^{-1}$; ${ }^{1} \mathrm{H}$ NMR (500 MHz, $\left.\mathrm{CDCl}_{3}\right): \delta=9.73(\mathrm{~d}, J=1.5 \mathrm{~Hz}, 1 \mathrm{H}$ ), 7.70-7.64 (m, $4 \mathrm{H}$ ), 7.47-7.35 (m, 6 H), 7.30-7.22 (m, 2 H), 6.90-6.85 (m, 2 H), 4.61 (d, $J=12.0$ Hz, 1 H), 4.60 (d, $J=$ $12.0 \mathrm{~Hz}, 1 \mathrm{H}$ ), 4.00-3.93 (m, $2 \mathrm{H}$ ), 3.88 (dt, $J=5.0,2.0 \mathrm{~Hz}, 1 \mathrm{H}$ ), 3.81 (s, $3 \mathrm{H}), 1.06$ (s, $9 \mathrm{H}) ;{ }^{13} \mathrm{C} \mathrm{NMR}$ (125 MHz, $\left.\mathrm{CDCl}_{3}\right): \delta=202.6,159.5,135.6,135.6,132.9,132.9,129.8,129.6,129.6,129.4,127.7$, 127.7, 113.9, 83.6, 72.2, 63.3, 55.3, 26.7, 19.2; HR-MS [Matrix-assisted laser desorption / ionization (MALDI)]: calcd for $\mathrm{C}_{27} \mathrm{H}_{32} \mathrm{O}_{4} \mathrm{SiNa}^{+}\left[\mathrm{M}+\mathrm{Na}^{+}\right]$: 471.1962, found 471.1957 .

Allyl alcohol 109. To a solution of (+)-(Ipc) ${ }_{2}$ Ballyl $^{7}$ (30.9 g, $95.4 \mathrm{mmol}$, 2.0 equiv) in $\mathrm{Et}_{2} \mathrm{O}$ (200 mL) at $-100{ }^{\circ} \mathrm{C}$ was added a pre-cooled $\left(-78{ }^{\circ} \mathrm{C}\right)$ solution of aldehyde $108(21.4 \mathrm{~g}, 47.7 \mathrm{mmol}, 1.0$ equiv) in TBDPSO $\mathrm{OH} \quad \mathrm{Et}_{2} \mathrm{O}(200 \mathrm{~mL})$. The solution was stirred at $-100{ }^{\circ} \mathrm{C}$ for $2 \mathrm{~h}$ and then quenched

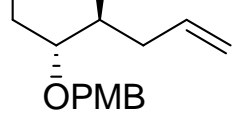

109 with EtOH (16 mL), $1 \mathrm{M} \mathrm{KOH} \mathrm{(200} \mathrm{mL),} \mathrm{and} \mathrm{30 \%} \mathrm{aqueous} \mathrm{H}_{2} \mathrm{O}_{2}(200 \mathrm{~mL})$, and allowed to warm to $25{ }^{\circ} \mathrm{C}$ over $14 \mathrm{~h}$. The biphasic mixture was extracted with $\mathrm{Et}_{2} \mathrm{O}(4 \times 200 \mathrm{~mL})$ and the combined organic extracts were dried $\left(\mathrm{Na}_{2} \mathrm{SO}_{4}\right)$, concentrated, and then purified by flash column chromatography (silica gel, EtOAc:hexanes $1: 8 \rightarrow 1: 6$ ) to afford a single isomer of allyl alcohol 109 (46.8 g, $95.4 \mathrm{mmol}, 100 \%$ ) as a colorless oil. 109: $R_{\mathrm{f}}=0.44$ (silica gel, EtOAc:hexanes 1:4); $[\alpha]_{\mathrm{D}}^{25}=-21.1\left(c=1.92, \mathrm{CHCl}_{3}\right)$; IR (film) $v_{\max }=3468,3070,2932,2852,1612$, 1428, $1109 \mathrm{~cm}^{-1}$; ${ }^{1} \mathrm{H}$ NMR (500 MHz, $\mathrm{CDCl}_{3}$ ): $\delta=7.73-7.68$ (m, 4 H), 7.48-7.36 (m, $6 \mathrm{H}$ ), 7.22-7.18 (m, 2 H), 6.90-6.86 (m, 2 H), 5.91-5.81 (m, 1 H), 5.15-5.08 (m, 2 H), 4.59 (d, J = 11.5 Hz, 1 H), 4.46 (d, $J=11.5$ Hz, 1 H), 3.93-3.84 (m, 3 H), 3.81 (s, 3 H), 3.48 (q, $J=5.5$ Hz, 1 H), 2.55 (d, $J=4.5$ Hz, 1 
H), 2.43-2.35 (m, $1 \mathrm{H}), 2.30-2.21(\mathrm{~m}, 1 \mathrm{H}), 1.09$ (s, $9 \mathrm{H}) ;{ }^{13} \mathrm{C}$ NMR (125 MHz, $\left.\mathrm{CDCl}_{3}\right): \delta=159.2$, 135.6, 135.6, 135.1, 133.1, 133.0, 130.5, 129.8, 129.4, 129.4, 127.8, 127.7, 117.4, 113.8, 80.9, 72.3, 71.7, 63.9, 55.3, 37.4, 26.8, 19.1; HR-MS [Matrix-assisted laser desorption / ionization (MALDI)]: calcd for $\mathrm{C}_{30} \mathrm{H}_{38} \mathrm{O}_{4} \mathrm{SiNa}^{+}\left[\mathrm{M}+\mathrm{Na}^{+}\right]$: 513.2431, found 513.2426.

Acrylate Ester 110. 4-DMAP (256 mg, 2.12 mmol, 0.1 equiv) and DCC (13.1 g, 63.6 mmol, 3.0 equiv) were added to a solution of allyl alcohol 109 (10.4 g, 21.2 mmol, 1.0 equiv) and acrylic acid (4.36 mL, to reflux for $4 \mathrm{~h}$ and then filtered through celite (the urea was washed with a $110 \mathrm{NaHCO}_{3}(250 \mathrm{~mL})$ was added, and the mixture was extracted with $\mathrm{Et}_{2} \mathrm{O}(5 \times 200$ $\mathrm{mL})$. The combined organic extracts were dried $\left(\mathrm{Na}_{2} \mathrm{SO}_{4}\right)$, concentrated and then purified by flash column chromatography (silica gel, EtOAc:hexanes 1:6) to provide acrylate ester 110 (8.97 g, 16.5 mmol, 78\%) as a colorless oil. 110: $R_{\mathrm{f}}=0.49$ (silica gel, EtOAc:hexanes 1:4); $[\alpha]_{\mathrm{D}}{ }^{25}=-9.4(c=1.46$, $\mathrm{CHCl}_{3}$ ); IR (film) $v_{\max }=3070,2932,2861,1725,1404,1110 \mathrm{~cm}^{-1} ;{ }^{1} \mathrm{H}$ NMR (500 MHz, $\left.\mathrm{CDCl}_{3}\right): \delta=$ 7.70-7.61 (m, 4 H), 7.45-7.32 (m, 6 H), 7.24-7.20 (m, 2 H), 6.86-6.82 (m, 2 H), 6.34 (dd, J = 17.5, 1.5 Hz, 1 H), 6.06 (dd, $J=17.5,10.5$ Hz, 1 H), 5.79 (dd, $J=10.5,1.5$ Hz, 1 H), 5.78-5.67 (m, 1 H), 5.28 (q, $J=5.5 \mathrm{~Hz}, 1 \mathrm{H}), 5.07-4.98$ (m, $2 \mathrm{H}), 4.58$ (d, $J=11.5 \mathrm{~Hz}, 1 \mathrm{H}), 4.52$ (d, $J=11.5 \mathrm{~Hz}, 1 \mathrm{H}), 3.80$ (s, 3 H), 3.75 (d, $J=5.0 \mathrm{~Hz}, 2 \mathrm{H}), 3.68$ (q, $J=5.0 \mathrm{~Hz}, 1 \mathrm{H}), 2.45$ (t, $J=7.0 \mathrm{~Hz}, 2 \mathrm{H}), 1.06$ (s, $9 \mathrm{H}) ;{ }^{13} \mathrm{C}$ NMR (125 MHz, $\left.\mathrm{CDCl}_{3}\right): \delta=165.3,159.2,135.6,135.6,133.9,133.2,130.5,130.5,129.7,129.6,129.3$, 128.6, 127.7, 127.7, 117.6, 113.7, 79.6, 72.8, 72.4, 63.2, 55.2, 34.13, 26.8, 19.1; HR-MS [Matrixassisted laser desorption / ionization (MALDI)]: calcd for $\mathrm{C}_{33} \mathrm{H}_{40} \mathrm{O}_{5} \mathrm{SiNa}^{+}\left[\mathrm{M}+\mathrm{Na}^{+}\right]$: 567.2537, found 567.2546.

Lactone 111. To a solution of acrylate ester 110 (13.8 g, $25.3 \mathrm{mmol}, 1.0$ equiv) in $\mathrm{CH}_{2} \mathrm{Cl}_{2}(2.60 \mathrm{~L})$ at 25 ${ }^{\circ} \mathrm{C}$ was added a solution of Grubbs' first generation catalyst 80 (1.9 g, 2.5 mmol, 0.1 equiv) in $\mathrm{CH}_{2} \mathrm{Cl}_{2}$ (200 mL). The reaction mixture was heated to reflux for $4 \mathrm{~h}$, then the solvent was removed, and the 
resulting residue was purified by flash column chromatography (silica gel, EtOAc:hexanes 1:4) to afford lactone 111 (11.9 g, $23.0 \mathrm{mmol}, 91 \%$ ) as a colorless oil. 111: $R_{\mathrm{f}}=0.31$ (silica gel, EtOAc:hexanes 1:4);

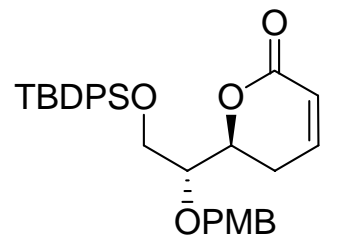
$[\alpha]_{\mathrm{D}}^{25}=-72.2\left(c=1.02, \mathrm{CHCl}_{3}\right) ;$ IR (film) $v_{\max }=2931,2857,1731,1512,1110$ $\mathrm{cm}^{-1} ;{ }^{1} \mathrm{H}$ NMR (500 MHz, $\left.\mathrm{CDCl}_{3}\right): \delta=7.68-7.62(\mathrm{~m}, 4 \mathrm{H}), 7.45-7.33(\mathrm{~m}, 6 \mathrm{H})$, 7.23-7.17 (m, 2 H), 6.86 (dd, $J=10.0,2.5 \mathrm{~Hz}, 1 \mathrm{H}$ ), 6.00 (ddd, $J=10.0,2.5,1.0$ 111 Hz, $1 \mathrm{H}), 4.69$ (dt, $J=12.0,4.0 \mathrm{~Hz}, 1 \mathrm{H}), 4.59$ (d, $J=11.5 \mathrm{~Hz}, 1 \mathrm{H}), 4.53$ (d, $J=$ 11.5 Hz, 1 H), 3.85-3.78 (m, 1 H), 3.80 (s, 3 H), 3.69 (dd, $J=12.0,7.5$ Hz, 1 H), 3.48 (dd, $J=11.0,7.5$ Hz, 1 H), 2.53 (ddt, $J=18.5,12.0,2.5 \mathrm{~Hz}, 1 \mathrm{H}), 2.07$ (ddd, $J=18.5,6.0,5.0 \mathrm{~Hz}, 1 \mathrm{H}), 1.05(\mathrm{~s}, 9 \mathrm{H}) ;{ }^{13} \mathrm{C}$ NMR (125 MHz, $\left.\mathrm{CDCl}_{3}\right): \delta=164.1,159.3,145.6,135.59,135.56,133.1,133.0,130.2,129.8,129.5$, 129.5, 127.8, 127.8, 121.0, 113.8, 79.1, 77.7, 72.9, 62.3, 55.3, 26.8, 24.2, 19.2; HR-MS [Matrix-assisted laser desorption / ionization (MALDI)]: calcd for $\mathrm{C}_{31} \mathrm{H}_{36} \mathrm{O}_{5} \mathrm{SiNa}^{+}\left[\mathrm{M}+\mathrm{Na}^{+}\right.$]: 539.2224, found 539.2209.

Methylated lactone 112. To a suspension of CuCN (192 mg, $2.15 \mathrm{mmol}, 1.5$ equiv) in $\mathrm{Et}_{2} \mathrm{O}(10 \mathrm{~mL})$ at $-78{ }^{\circ} \mathrm{C}$ was added MeLi (3.4 mL, 1.5 $\mathrm{M}$ in $\mathrm{Et}_{2} \mathrm{O}, 4.3 \mathrm{mmol}, 3.0$ equiv). The mixture was allowed to TBDPSO warm to $0{ }^{\circ} \mathrm{C}$ over $1 \mathrm{~h}$ and was then re-cooled to $-78^{\circ} \mathrm{C}$. A pre-cooled $\left(-78^{\circ} \mathrm{C}\right)$ solution of lactone 111 (0.73 g, $1.43 \mathrm{mmol}, 1.0$ equiv) in $\mathrm{Et}_{2} \mathrm{O}$ (10 mL) was then added dropwise. After 20 min the mixture was quenched with a 10:1 mixture of saturated aqueous $\mathrm{NH}_{4} \mathrm{Cl}: 25 \% \mathrm{NH}_{4} \mathrm{OH}(50 \mathrm{~mL})$, and the resulting biphasic mixture was stirred for an additional 2.5 h. The mixture was then extracted with $\mathrm{Et}_{2} \mathrm{O}(3 \times 30$ $\mathrm{mL})$ and the combined organic extracts were dried $\left(\mathrm{Na}_{2} \mathrm{SO}_{4}\right)$, concentrated, and then purified by flash column chromatography (silica gel, EtOAc:hexanes 1:1) to give a single isomer of methylated lactone $112(0.73 \mathrm{~g}, 1.38 \mathrm{mmol}, 95 \%)$ as a colorless oil. 112: $R_{\mathrm{f}}=0.35$ (silica gel, EtOAc:hexanes $\left.1: 4\right) ;[\alpha]_{\mathrm{D}}{ }^{25}=$ $-3.0\left(c=1.04, \mathrm{CHCl}_{3}\right)$; IR (film) $v_{\max }=2955,2860,1741,1464,1109 \mathrm{~cm}^{-1} ;{ }^{1} \mathrm{H} \mathrm{NMR}(500 \mathrm{MHz}$, $\left.\mathrm{CDCl}_{3}\right): \delta=7.65-7.60$ (m, $\left.4 \mathrm{H}\right)$, 7.44-7.36 (m, $\left.6 \mathrm{H}\right), 7.20-7.15$ (m, $\left.2 \mathrm{H}\right), 6.85-6.81$ (m, 2 H), 4.70-4.65 (m, 1 H), 4.53 (d, $J=11.0$ Hz, 1 H), 4.49 (d, $J=11.0$ Hz, 1 H), 3.80 (s, 3 H), 3.82-3.74 (m, 2 H), 3.69 (m, $1 \mathrm{H}$ ), 2.56-2.51 (m, $1 \mathrm{H}), 2.13$ (dd, $J=17.0,9.0 \mathrm{~Hz}, 1 \mathrm{H}), 2.00$ (ddd, $J=14.0,9.0,6.0 \mathrm{~Hz}, 1 \mathrm{H}$ ), 1.53 (ddd, $J=14.0,7.0,5.0 \mathrm{~Hz}, 1 \mathrm{H}), 1.30-1.22$ (m, $1 \mathrm{H}), 1.05$ (s, $9 \mathrm{H}), 1.02$ (d, $J=6.5 \mathrm{~Hz}, 3 \mathrm{H}) ;{ }^{13} \mathrm{C}$ 
NMR (125 MHz, $\left.\mathrm{CDCl}_{3}\right): \delta=171.9,159.2,135.57,135.55,133.1,133.0,130.2,129.8,129.6,129.6$, 127.8, 127.8, 113.7, 79.8, 76.9, 73.1, 62.6, 55.2, 37.7, 29.3, 26.8, 23.9, 21.0, 19.2; HR-MS [Matrixassisted laser desorption / ionization (MALDI)]: calcd for $\mathrm{C}_{32} \mathrm{H}_{40} \mathrm{O}_{5} \mathrm{SiNa}^{+}\left[\mathrm{M}+\mathrm{Na}^{+}\right]$: 555.2537, found 555.2540.

Primary Alcohol 113. To a solution of methylated lactone 112 (21.2 g, 39.8 mmol, 1.0 equiv) in THF $(120 \mathrm{~mL})$ at $0{ }^{\circ} \mathrm{C}$ was added TBAF $(59.7 \mathrm{~mL}, 1.0 \mathrm{M}$ in THF, $59.7 \mathrm{mmol}, 1.5$ equiv). The solution was

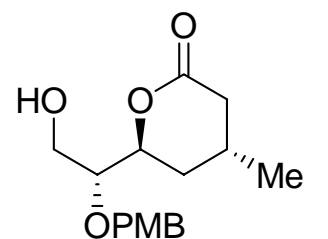

113 stirred at $25{ }^{\circ} \mathrm{C}$ for 30 min then quenched with saturated aqueous $\mathrm{NH}_{4} \mathrm{Cl}(800 \mathrm{~mL})$. The resulting biphasic mixture was extracted with EtOAc $(4 \times 200 \mathrm{~mL})$ and the combined organic extracts were dried $\left(\mathrm{Na}_{2} \mathrm{SO}_{4}\right)$, concentrated, and then purified by flash column chromatography (silica gel, EtOAc:hexanes 4:1) to afford primary alcohol 113 (10.2 g, $34.7 \mathrm{mmol}, 87 \%)$ as a colorless oil. 113: $R_{\mathrm{f}}=0.16$ (silica gel, EtOAc:hexanes 1:1); $[\alpha]_{\mathrm{D}}{ }^{25}=+5.2\left(c=1.16, \mathrm{CHCl}_{3}\right) ; \mathrm{IR}($ film $) v_{\max }=3442,2957,1733,1460,1079 \mathrm{~cm}^{-1} ;{ }^{1} \mathrm{H}$ NMR (500 $\left.\mathrm{MHz}, \mathrm{CDCl}_{3}\right): \delta=7.26(\mathrm{~d}, J=9.0 \mathrm{~Hz}, 2 \mathrm{H}), 6.89(\mathrm{~d}, J=9.0 \mathrm{~Hz}, 2 \mathrm{H}), 4.61$ (d, $\left.J=11.5 \mathrm{~Hz}, 1 \mathrm{H}\right), 4.57$ (d, $J=11.5 \mathrm{~Hz}, 1 \mathrm{H}), 4.52$ (ddd, $J=8.0,6.5,5.0 \mathrm{~Hz}, 1 \mathrm{H}), 3.81$ (s, $3 \mathrm{H}), 3.80-3.72$ (m, $2 \mathrm{H}), 3.62-3.58$ (m, 1 H), 2.58-2.52 (m, 1 H), 2.15 (dd, $J=17.0,9.0$ Hz, 1 H), 2.14-2.08 (m, 1 H), 1.97 (ddd, $J=14.0$, 8.5, $6.0 \mathrm{~Hz}, 1 \mathrm{H}), 1.82$ (t, $J=6.5 \mathrm{~Hz}, 1 \mathrm{H}), 1.63$ (ddd, $J=14.0,6.0,5.0 \mathrm{~Hz}, 1 \mathrm{H}), 1.05$ (d, $J=7.0 \mathrm{~Hz}, 3$ $\mathrm{H}) ;{ }^{13} \mathrm{C}$ NMR (125 MHz, $\left.\mathrm{CDCl}_{3}\right): \delta=171.2,159.5,129.8,129.7,114.0,79.5,76.3,72.7,60.7,55.3$, 37.7, 30.7, 23.8, 21.1; HR-MS [Matrix-assisted laser desorption / ionization (MALDI)]: calcd for $\mathrm{C}_{16} \mathrm{H}_{22} \mathrm{O}_{5} \mathrm{Na}^{+}\left[\mathrm{M}+\mathrm{Na}^{+}\right]$: 317.1359, found 317.1359.

Aldehyde 114. To a solution of primary alcohol 113 (10.2 g, $34.7 \mathrm{mmol}, 1.0$ equiv) in $\mathrm{CH}_{2} \mathrm{Cl}_{2}(150 \mathrm{~mL})$ at $25^{\circ} \mathrm{C}$ was added pyridine (28.0 mL, $346 \mathrm{mmol}, 10$ equiv), and Dess-Martin periodinane (20.6 g, 48.6<smiles>CCCCC(C)OC1CC(C)CC(=O)O1</smiles>

114 mmol, 1.5 equiv). The reaction was stirred at $25{ }^{\circ} \mathrm{C}$ for $2 \mathrm{~h}$ and then quenched with saturated aqueous $\mathrm{Na}_{2} \mathrm{~S}_{2} \mathrm{O}_{3}(200 \mathrm{~mL})$ and saturated aqueous $\mathrm{NaHCO}_{3}(200 \mathrm{~mL})$. The biphasic mixture was extracted with $\mathrm{CH}_{2} \mathrm{Cl}_{2}(4 \times 200 \mathrm{~mL})$ and the combined organic extracts were dried $\left(\mathrm{Na}_{2} \mathrm{SO}_{4}\right)$, concentrated, and then purified by flash 
column chromatography (silica gel, EtOAc:hexanes 2:1) to afford aldehyde 114 (9.33 g, 31.9 mmol, 92\%) as a colorless oil. 114: $R_{\mathrm{f}}=0.37$ (silica gel, EtOAc:hexanes $\left.1: 1\right) ;[\alpha]_{\mathrm{D}}{ }^{25}=-44.5(c=0.28$, $\mathrm{CHCl}_{3}$ ); IR (film) $v_{\max }=2958,1734,1612,1249,1079 \mathrm{~cm}^{-1} ;{ }^{1} \mathrm{H}$ NMR $\left(500 \mathrm{MHz}, \mathrm{CDCl}_{3}\right): \delta=9.64(\mathrm{~d}$, $J=1.1 \mathrm{~Hz}, 1 \mathrm{H}), 7.28$ (d, $J=8.5 \mathrm{~Hz}, 2 \mathrm{H}), 6.89$ (d, $J=8.5 \mathrm{~Hz}, 2 \mathrm{H}), 4.77(\mathrm{dt}, J=9.0,5.0 \mathrm{~Hz}, 1 \mathrm{H}), 4.66$ (s, 2 H), 4.05 (dd, $J=4.5,1.1$ Hz, 1 H), 3.81 (s, 3 H), 2.62-2.57 (m, 1 H), 2.24-2.15 (m, 2 H), 2.04 (ddd, $J=14.5,9.0,6.0 \mathrm{~Hz}, 1 \mathrm{H}), 1.48$ (ddd, $J=14.5,6.0,5.0 \mathrm{~Hz}, 1 \mathrm{H}), 1.05$ (d, $J=6.5 \mathrm{~Hz}, 3 \mathrm{H}) ;{ }^{13} \mathrm{C}$ NMR (125 MHz, $\left.\mathrm{CDCl}_{3}\right): \delta=201.2,171.0,159.7,130.1,128.6,114.0,83.5,76.6,73.7,55.3,37.5$, 29.7, 23.8, 20.8; HR-MS [Matrix-assisted laser desorption / ionization (MALDI)]: calcd for $\mathrm{C}_{16} \mathrm{H}_{20} \mathrm{O}_{5} \mathrm{Na}^{+}\left[\mathrm{M}+\mathrm{Na}^{+}\right]$: 315.1203, found 315.1196.

Aldol Product 115. To a solution of methyl ketone 23 (1.33 g, 7.86 mmol, 1.1 equiv) in $\mathrm{CH}_{2} \mathrm{Cl}_{2}(30$ $\mathrm{mL}$ ) at $0{ }^{\circ} \mathrm{C}$ was added chlorodicyclohexylborane (2.04 mL, $9.3 \mathrm{mmol}, 1.3$ equiv), followed by $N, N-$

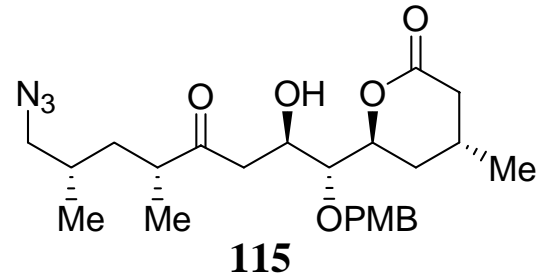

115 diisopropylethylamine (1.86 mL, $10.7 \mathrm{mmol}, 1.5$ equiv). After $2 \mathrm{~h}$ at 0 ${ }^{\circ} \mathrm{C}$, the mixture was cooled to $-78{ }^{\circ} \mathrm{C}$ and a pre-cooled $\left(-78{ }^{\circ} \mathrm{C}\right)$ solution of aldehyde 114 in $\mathrm{CH}_{2} \mathrm{Cl}_{2}(30 \mathrm{~mL})$ was added via cannula. After $3.5 \mathrm{~h}$ at $-78{ }^{\circ} \mathrm{C}$ the reaction mixture was quenched with $\mathrm{MeOH}$ (5 $\mathrm{mL}$ ), a pH 7 buffered saline solution $(40 \mathrm{~mL})$, and $30 \%$ aqueous $\mathrm{H}_{2} \mathrm{O}_{2}(4 \mathrm{~mL})$. The mixture was stirred vigorously and allowed to slowly warm to $25{ }^{\circ} \mathrm{C}$ over a period of $12 \mathrm{~h}$. The resulting biphasic mixture was extracted with $\mathrm{CH}_{2} \mathrm{Cl}_{2}(4 \times 100 \mathrm{~mL})$ and the combined organic layers were dried $\left(\mathrm{MgSO}_{4}\right)$, concentrated, and then purified by flash column chromatography (silica gel, EtOAc:hexanes 1:4 $\rightarrow$ 1:1) to afford a single isomer of aldol product 115 (3.14 g, 93\%) as a colorless oil. 115: $R_{\mathrm{f}}=0.47$ (silica gel, EtOAc:hexanes 1:1); $[\alpha]_{\mathrm{D}}^{25}=+36.8\left(c=1.05, \mathrm{CHCl}_{3}\right)$; IR (film) $v_{\max }=3458,2960,2930,2097,1732$, 1708, 1459, $1087 \mathrm{~cm}^{-1}$; ${ }^{1} \mathrm{H}$ NMR (500 MHz, $\left.\mathrm{CDCl}_{3}\right): \delta=7.25$ (d, $\left.J=8.5 \mathrm{~Hz}, 2 \mathrm{H}\right), 6.88(\mathrm{~d}, J=8.5 \mathrm{~Hz}$, $2 \mathrm{H}), 4.75$ (dt, $J=9.0,5.0 \mathrm{~Hz}, 1 \mathrm{H}), 4.70$ (d, $J=11.4$ Hz, $1 \mathrm{H}), 4.55$ (d, $J=11.4 \mathrm{~Hz}, 1 \mathrm{H}), 4.05-4.00$ (m, $1 \mathrm{H}), 3.78$ (s, $3 \mathrm{H}), 3.66$ (dd, $J=7.5,4.0 \mathrm{~Hz}, 1 \mathrm{H}), 3.39-3.31$ (m, $1 \mathrm{H}), 3.15$ (dd, $J=12.0,6.0 \mathrm{~Hz}, 1 \mathrm{H}$ ), 3.12 (dd, $J=12.0,6.0 \mathrm{~Hz}, 1 \mathrm{H}), 2.94$ (dd, $J=18.0,2.0 \mathrm{~Hz}, 1 \mathrm{H}), 2.68-2.59$ (m, $2 \mathrm{H}), 2.50$ (dd, $J=18.0$, 9.0 Hz, 1 H), 2.28-2.22 (m, 2 H), 2.15 (ddd, $J=14.0,9.0,5.5$ Hz, 1 H), 1.85 (ddd, $J=13.5,8.5,6.0$ Hz, 
$1 \mathrm{H}), 1.74-1.63$ (m, $2 \mathrm{H}), 1.18$ (ddd, $J=13.5,8.0,6.0 \mathrm{~Hz}, 1 \mathrm{H}), 1.15$ (d, $J=7.0 \mathrm{~Hz}, 3 \mathrm{H}), 1.14$ (d, $J=$ $7.0 \mathrm{~Hz}, 3 \mathrm{H}), 1.01$ (d, $J=7.0 \mathrm{~Hz}, 3 \mathrm{H}) ;{ }^{13} \mathrm{C} \mathrm{NMR}\left(125 \mathrm{MHz}, \mathrm{CDCl}_{3}\right): \delta=215.8,172.1,159.4,130.0$, 129.9, 113.8, 80.9, 77.3, 74.2, 67.4, 57.4, 55.2, 44.4, 43.3, 37.6, 37.1, 31.4, 28.9, 23.9, 21.0, 17.9, 17.1; HR-MS [Matrix-assisted laser desorption / ionization (MALDI)]: calcd for $\mathrm{C}_{24} \mathrm{H}_{35} \mathrm{~N}_{3} \mathrm{O}_{6} \mathrm{Na}^{+}\left[\mathrm{M}+\mathrm{Na}^{+}\right]$: 484.2418, found 484.2416.

Benzoate 116. To a solution of aldol product 115 (120.3 mg, $0.261 \mathrm{mmol}, 1.0$ equiv) in pyridine (1.6 $\mathrm{mL}$ ) at $0{ }^{\circ} \mathrm{C}$ was added benzoyl chloride (110 mg, $0.782 \mathrm{mmol}, 3.0$ equiv). The solution was stirred for 4

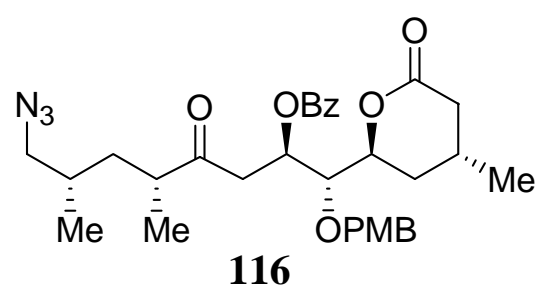
h at $0{ }^{\circ} \mathrm{C}$ and then quenched with saturated aqueous $\mathrm{NaHCO}_{3}(10 \mathrm{~mL})$. The biphasic mixture was extracted with $\mathrm{CH}_{2} \mathrm{Cl}_{2}(3 \times 20 \mathrm{~mL})$ and the combined organic layers were dried $\left(\mathrm{Na}_{2} \mathrm{SO}_{4}\right)$, concentrated, and then purified by flash column chromatography (silica gel, EtOAc:hexanes 1:2) to afford benzoate 116 (129 mg, 88\%) as a white crystalline solid. 116: $R_{\mathrm{f}}=0.61$ (silica gel, EtOAc:hexanes 1:1); $[\alpha]_{\mathrm{D}}{ }^{25}=+9.1\left(\mathrm{c}=0.35, \mathrm{CHCl}_{3}\right)$; mp $=94-95{ }^{\circ} \mathrm{C}$; IR (film) $v_{\max }=2959,2931$, 2097, 1719, 1707, 1458, $1097 \mathrm{~cm}^{-1}$; ${ }^{1} \mathrm{H}$ NMR (500 MHz, $\mathrm{CDCl}_{3}$ ): $\delta=7.98-7.96$ (m, $2 \mathrm{H}$ ), 7.60-7.55 (m, 1 H), 7.47-7.40 (m, 2 H), 7.25 (d, $J=8.6$ Hz, 2 H), 6.85 (d, $J=8.6$ Hz, 2 H), 5.77 (dt, $J=7.4,4.8$ Hz, 1 H), 4.69 (d, $J=11.2 \mathrm{~Hz}, 1 \mathrm{H}), 4.65$ (d, $J=11.2 \mathrm{~Hz}, 1 \mathrm{H}), 4.59$ (dt, $J=7.3,4.8 \mathrm{~Hz}, 1 \mathrm{H}), 3.92$ (t, $J$ $=4.8 \mathrm{~Hz}, 1 \mathrm{H}), 3.79$ (s, $3 \mathrm{H}), 3.15$ (dd, $J=12.1,5.2 \mathrm{H}, 1 \mathrm{H}), 3.13-3.08$ (m, $1 \mathrm{H}), 3.07$ (dd, $J=12.1,6.6$ Hz, 1 H), 2.95 (dd, $J=17.4,7.7$ Hz, 1 H), 2.64-2.51 (m, 2 H), 2.15-2.03 (m, 3 H), 1.76 (ddd, $J=13.9$, 8.1, $6.1 \mathrm{~Hz}, 1 \mathrm{H}), 1.69-1.60$ (m, $2 \mathrm{H}), 1.08$ (ddd, $J=13.9,8.1,6.3 \mathrm{~Hz}, 1 \mathrm{H}), 1.07$ (d, J = 7.0 Hz, $3 \mathrm{H}$ ), 1.00 (d, $J=7.0 \mathrm{~Hz}, 3 \mathrm{H}), 0.91$ (d, $J=7.0 \mathrm{~Hz}, 3 \mathrm{H}) ;{ }^{13} \mathrm{C}$ NMR $\left(125 \mathrm{MHz}, \mathrm{CDCl}_{3}\right): \delta=210.6,171.2$, 165.4, 159.5, 133.3, 130.0, 130.0, 129.6, 129.5, 128.4, 113.8, 79.4, 76.7, 73.4, 64.5, 57.4, 55.2, 44.1, 41.6, 37.6, 37.1, 31.4, 30.0, 23.7, 20.9, 18.0, 16.9; HR-MS [Matrix-assisted laser desorption / ionization (MALDI)]: calcd for $\mathrm{C}_{31} \mathrm{H}_{39} \mathrm{~N}_{3} \mathrm{O}_{7} \mathrm{Na}^{+}\left[\mathrm{M}+\mathrm{Na}^{+}\right]$: 588.2680, found 588.2672.

Alcohol 102. To a solution of benzoate 116 (141 mg, $0.249 \mathrm{mmol}, 1.0$ equiv) in $\mathrm{CH}_{2} \mathrm{Cl}_{2}(3 \mathrm{~mL})$ at $0{ }^{\circ} \mathrm{C}$ was added water $(0.2 \mathrm{~mL})$ and DDQ (85.0 mg, $0.374 \mathrm{mmol}, 1.5$ equiv). The heterogeneous mixture was 
stirred vigorously for $3 \mathrm{~h}$ and then quenched with $1 \mathrm{M} \mathrm{NaOH}(30 \mathrm{~mL})$. The resulting biphasic mixture was extracted with $\mathrm{CH}_{2} \mathrm{Cl}_{2}(3 \times 20 \mathrm{~mL})$ and the combined organic layers were dried $\left(\mathrm{Na}_{2} \mathrm{SO}_{4}\right)$,

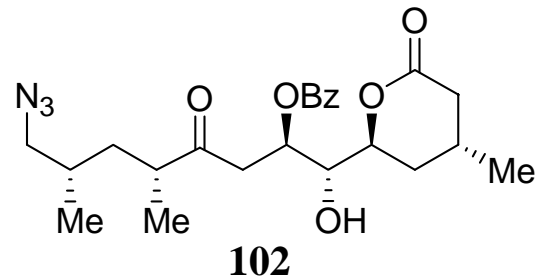
concentrated, and then purified by flash column chromatography (silica gel, EtOAc:hexanes 1:2) to afford alcohol 102 (106.3 mg, $0.243 \mathrm{mmol}$, 96\%) as a colorless oil. 102: $R_{\mathrm{f}}=0.41$ (silica gel, EtOAc:hexanes 1:1); $[\alpha]_{\mathrm{D}}^{25}=+14.1\left(c=0.52, \mathrm{CHCl}_{3}\right) ; \mathrm{IR}($ film $) v_{\max }=3448,2961,2931$, 2097, 1734, 1710, 1458, $1112 \mathrm{~cm}^{-1}$; ${ }^{1} \mathrm{H}$ NMR (500 MHz, $\mathrm{CDCl}_{3}$ ): $\delta=8.05-8.00$ (m, $2 \mathrm{H}$ ), 7.63-7.57 (m, 1 H), 7.50-7.40 (m, 2 H), 5.55 (d, $J=5.9$ Hz, 1 H), 4.45 (m, 1 H), 4.42 (dd, $J=4.1,1.9$ Hz, 1 H), 3.18-3.09 (m, 2 H), 3.05 (dd, J = 17.6, 6.6 Hz, 1 H), 2.77 (br s, 1 H), 2.67 (m, 1 H), 2.65-2.54 (m, 2 H), 2.36 (dd, $J$ = 14.3, 6.6 Hz, 1 H), 2.30-2.11 (m, 3 H), 1.91-1.60 (m, 3 H), 1.12 (d, $J=6.6$ Hz, 3 H), 1.06 (d, $J=6.6 \mathrm{~Hz}, 3 \mathrm{H}), 1.01$ (d, $J=6.6 \mathrm{~Hz}, 3 \mathrm{H}) ;{ }^{13} \mathrm{C} \mathrm{NMR}\left(125 \mathrm{MHz}, \mathrm{CDCl}_{3}\right): \delta=208.5,172.5,165.9$, 133.3, 130.0, 129.6, 128.4, 79.4, 73.9, 65.2, 57.4, 53.8, 44.1, 42.8, 37.9, 37.1, 31.4, 31.1, 23.7, 20.1, 18.0, 16.7; HR-MS [Matrix-assisted laser desorption / ionization (MALDI)]: calcd for $\mathrm{C}_{23} \mathrm{H}_{31} \mathrm{~N}_{3} \mathrm{O}_{6} \mathrm{Na}^{+}$ $\left[\mathrm{M}+\mathrm{Na}^{+}\right]:$468.2105, found 468.2113 .

Methyl Acetal 117. To a solution of alcohol 102 (109 mg, $0.245 \mathrm{mmol}, 1.0$ equiv) in $\mathrm{MeOH}$ (4 mL) at $25{ }^{\circ} \mathrm{C}$ was added PPTS (12 mg, $0.049 \mathrm{mmol}, 0.2$ equiv). The resulting clear solution was stirred for $2 \mathrm{~h}$

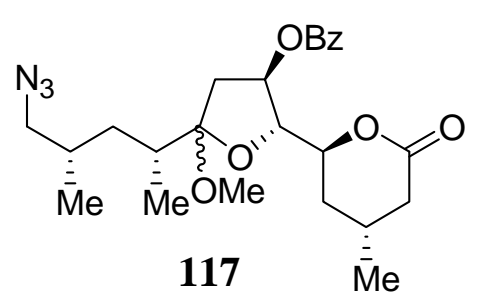
at $25{ }^{\circ} \mathrm{C}$ and then quenched with saturated aqueous $\mathrm{NaHCO}_{3}(30 \mathrm{~mL})$ and extracted with $\mathrm{CH}_{2} \mathrm{Cl}_{2}(3 \times 25 \mathrm{~mL})$. The combined organic extracts were dried $\left(\mathrm{Na}_{2} \mathrm{SO}_{4}\right)$, concentrated, and then purified by flash column chromatography (silica gel, EtOAc:hexanes 1:4) to afford a 2:1 ratio of acetals 117 (105 mg, $0.228 \mathrm{mmol}, 93 \%$ ) as a colorless oil. 117 (mixture of epimers ca 2:1, major epimer listed): $R_{\mathrm{f}}=0.55$ (silica gel, EtOAc:hexanes 1:1); IR (film) $v_{\max }=2960,2929,2097,1734$, 1710, 1458, $1112 \mathrm{~cm}^{-1} ;{ }^{1} \mathrm{H}$ NMR (500 MHz, $\left.\mathrm{CDCl}_{3}\right): \delta=8.05-8.00$ (m, $2 \mathrm{H}$ ), 7.60-7.55 (m, $1 \mathrm{H}$ ), 7.47-7.42 (m, 2 H), 5.38 (ddd, $J=8.7,4.0,2.2 \mathrm{~Hz}, 1 \mathrm{H}$ ), 4.61 (dt, $J=9.9,4.0 \mathrm{~Hz}, 1 \mathrm{H}$ ), 4.28 (t, $J=4.0$ Hz, 1 H), 3.30 (dd, $J=12.1,4.8$ Hz, 1 H), 3.27 (s, 3 H), 3.28-3.24 (m, 1 H), 3.14 (dd, $J=12.1,6.6$ Hz, 1 H), 2.61 (dd, $J=16.2,5.2 \mathrm{~Hz}, 1$ H), 2.41 (dd, $J=14.7,8.4 \mathrm{~Hz}, 1$ H), 2.41-2.01 (m, 3 H), 1.99 (dd, $J=$ 
14.7, $2.2 \mathrm{~Hz}, 1 \mathrm{H}), 1.84-1.72$ (m, 2 H), 1.66-1.59 (m, 2 H), 1.13 (d, $J=6.6 \mathrm{~Hz}, 3 \mathrm{H}), 1.00$ (d, $J=6.6$ $\mathrm{Hz}, 3 \mathrm{H}), 0.97$ (d, $J=6.6 \mathrm{~Hz}, 3 \mathrm{H}) ;{ }^{13} \mathrm{C}$ NMR $\left(125 \mathrm{MHz}, \mathrm{CDCl}_{3}\right): \delta=172.8,166.6,133.7,130.1,130.0$, 128.9, 112.8, 84.6, 77.0, 74.6, 57.1, 48.3, 38.8, 37.8, 36.8, 33.5, 31.4, 30.8, 24.3, 21.6, 20.0, 15.4; HRMS [Matrix-assisted laser desorption / ionization (MALDI)]: calcd for $\mathrm{C}_{24} \mathrm{H}_{33} \mathrm{~N}_{3} \mathrm{O}_{6} \mathrm{Na}^{+}\left[\mathrm{M}+\mathrm{Na}^{+}\right]$: 482.2262, found 482.2281 .

Teoc-Protected Amine 101. To a solution of methyl acetal 117 (2.01 g, $4.37 \mathrm{mmol}, 1.0$ equiv) in EtOAc $(150 \mathrm{~mL})$ at $25^{\circ} \mathrm{C}$ was added $\mathrm{Pd} / \mathrm{C}(0.50 \mathrm{~g}, 25 \% \mathrm{w} / \mathrm{w})$. The flask was then purged with $\mathrm{H}_{2}$ three

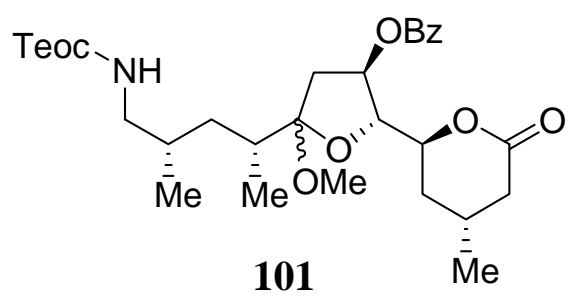
times and the reaction mixture was stirred at $25{ }^{\circ} \mathrm{C}$ under a $\mathrm{H}_{2}$ atmosphere for $7 \mathrm{~h}$. The reaction mixture was then filtered through a pad of celite and washed with EtOAc $(150 \mathrm{~mL})$ and the obtained primary amine 118 was used in the next step without any further purification. To the obtained primary amine 118 in EtOAc $(300 \mathrm{~mL})$ at $25^{\circ} \mathrm{C}$ was added $\mathrm{Et}_{3} \mathrm{~N}(3.05 \mathrm{~mL}$, $21.9 \mathrm{mmol}, 5.0$ equiv) and Teoc-O-( $\left.p-\mathrm{NO}_{2} \mathrm{Ph}\right)^{8}$ (4.96 g, $17.5 \mathrm{mmol}, 4.0$ equiv) and the obtained solution was stirred at $25^{\circ} \mathrm{C}$ for $15 \mathrm{~h}$. When complete the reaction mixture was concentrated and then purified by flash column chromatography (silica gel, EtOAc:hexanes 1:2) to provide a 2:1 ratio of Teoc-protected amines 101 (2.03 g, 3.54 mmol, 80\% over two steps) as a colorless oil. 101 (mixture of epimers ca 2:1, major epimer listed): $R_{\mathrm{f}}=0.55$ (silica gel, EtOAc:hexanes 1:1); IR (film) $v_{\max }=3383,2956,1742$, 1712, 1522, 1451, 1279, $1249 \mathrm{~cm}^{-1}$; ${ }^{1} \mathrm{H}$ NMR (600 MHz, $\mathrm{CDCl}_{3}$ ): $\delta=8.01-7.98$ (m, $2 \mathrm{H}$ ), 7.55-7.53 (m, 1 H), 7.43-7.40 (m, 2 H), 5.38-5.36 (m, 1 H), 4.77-4.73 (m, 1 H), 4.60-4.56 (m, 1 H), 4.14-4.08 (m, 2 H), 3.23 (s, 3 H), 2.90-2.85 (m, 1 H), 2.63-2.57 (m, 1 H), 2.41-2.37 (m, 1 H), 2.26-1.94 (m, 8 H), 1.77-1.72 (m, 1 H), 1.61-1.59 (m, 1 H), 1.53-1.49 (m, 1 H), 1.10 (d, $J=7.0$ Hz, 3 H), 0.96 (d, $J=7.0$ $\mathrm{Hz}, 3 \mathrm{H}), 0.90$ (d, $J=7.0 \mathrm{~Hz}, 3 \mathrm{H}), 0.90-0.86(\mathrm{~m}, 2 \mathrm{H}), 0.01$ (s, $9 \mathrm{H}) ;{ }^{13} \mathrm{C}$ NMR $\left(150 \mathrm{MHz}, \mathrm{CDCl}_{3}\right): \delta=$ 171.4, 166.1, 156.9, 133.2, 129.7, 129.6, 128.3, 112.3, 84.3, 84.0, 76.4, 75.7, 74.2, 62.8, 47.9, 38.3, 37.3, 36.5, 33.2, 30.9, 30.3, 23.7, 21.1, 17.7, 15.0, -1.6; HR-MS [Matrix-assisted laser desorption / ionization (MALDI)]: calcd for $\mathrm{C}_{30} \mathrm{H}_{47} \mathrm{NO}_{8} \mathrm{SiNa}^{+}\left[\mathrm{M}+\mathrm{Na}^{+}\right]:$600.2963, found 600.2966 . 
Tricyclic Aminal 119. To a solution of Teoc-protected amine 101 (300 mg, $0.52 \mathrm{mmol}, 1.0$ equiv) in $\mathrm{CH}_{3} \mathrm{CN}(45 \mathrm{~mL})$ at $25^{\circ} \mathrm{C}$ was added $\mathrm{Nd}(\mathrm{OTf})_{3}$ (30 $\mathrm{mg}, 0.05 \mathrm{mmol}, 0.1$ equiv). The reaction mixture was

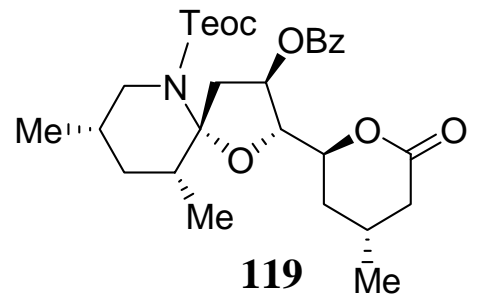
allowed to stir for $15 \mathrm{~min}$ and then quenched with saturated aqueous $\mathrm{NaHCO}_{3}(50 \mathrm{~mL})$. The resulting biphasic mixture was extracted with EtOAc $(3 \times 50 \mathrm{~mL})$ and the combined organic extracts were dried $\left(\mathrm{Na}_{2} \mathrm{SO}_{4}\right)$, concentrated, and then purified by flash column chromatography (silica gel, EtOAc:hexanes 2:3) to afford a single isomer of tricyclic aminal 119 (240 mg, 0.42 mmol, 81\%) as a colorless oil. 119: $R_{\mathrm{f}}=0.70$ (silica gel, EtOAc:hexanes 1:1); $[\alpha]_{\mathrm{D}}{ }^{25}=-13.1(c=1.14$, $\mathrm{CHCl}_{3}$ ); IR (film) $v_{\max }=3383,2956,1742,1712,1522,1451,1279,1249 \mathrm{~cm}^{-1} ;{ }^{1} \mathrm{H} \mathrm{NMR}(600 \mathrm{MHz}$, $\left.\mathrm{CDCl}_{3}\right): \delta=8.00-7.99$ (m, $\left.2 \mathrm{H}\right), 7.56-7.53$ (m, $\left.1 \mathrm{H}\right), 7.43-7.40$ (m, $\left.2 \mathrm{H}\right)$, 5.66-5.64 (m, $\left.1 \mathrm{H}\right), 4.46$ (dt, $J$ = 9.0, 4.8 Hz, $1 \mathrm{H}), 4.27$ (dd, $J=7.8,1.8 \mathrm{~Hz}, 1 \mathrm{H}), 4.15$ (t, $J=9.6 \mathrm{~Hz}, 2 \mathrm{H}), 3.77-3.75$ (m, $1 \mathrm{H}), 3.38$ (dd, $J=16.2,6.8 \mathrm{~Hz}, 1 \mathrm{H}), 2.96$ (t, $J=12.0 \mathrm{~Hz}, 1 \mathrm{H}), 2.61$ (dd, $J=16.8,6.0 \mathrm{~Hz}, 1 \mathrm{H}), 2.56$ (dd, $J=$ 16.8, 2.4 Hz, 1 H), 2.26-2.22 (m, 1 H), 2.15 (dd, $J=16.8,9.0$ Hz, 1 H), 2.03-1.97 (m, 2 H), 1.73-1.69 (m, 1 H), 1.63-1.55 (m, 2 H), 1.10 (d, J = 7.0 Hz, 3 H), 1.05-0.98 (m, 3 H), 0.97 (d, J = 7.0 Hz, 3 H), 0.84 (d, $J=7.0 \mathrm{~Hz}, 3 \mathrm{H}), 0.01$ (s, $9 \mathrm{H}) ;{ }^{13} \mathrm{C} \mathrm{NMR}\left(150 \mathrm{MHz}, \mathrm{CDCl}_{3}\right): \delta=171.0,165.7,156.4,133.0$, 129.8, 129.6, 128.3, 99.3, 85.7, 76.5, 75.8, 63.5, 60.3, 50.2, 40.6, 37.8, 37.6, 31.7, 30.9, 23.4, 21.1, 18.7, 17.1, 14.2, -1.5; HR-MS [Matrix-assisted laser desorption / ionization (MALDI)]: calcd for $\mathrm{C}_{29} \mathrm{H}_{43} \mathrm{NO}_{7} \mathrm{SiNa}^{+}\left[\mathrm{M}+\mathrm{Na}^{+}\right]:$568.2701, found 568.2709.

Lactone Alcohol 121. To a solution of tricyclic aminal 119 (4.02 g, 7.37 mmol, 1.0 equiv) in toluene (95 mL) at $-78{ }^{\circ} \mathrm{C}$ was added DIBAL-H (29.5 mL, $1.00 \mathrm{M}$ in $\mathrm{CH}_{2} \mathrm{Cl}_{2}, 29.5 \mathrm{mmol}, 4.0$ equiv). After 30

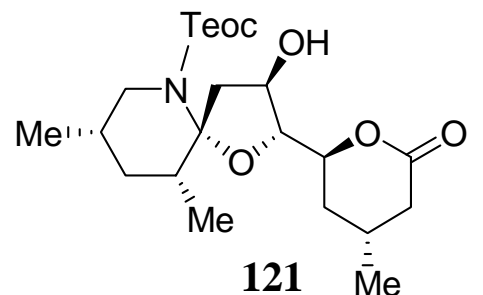
min the reaction mixture was quenched with $\mathrm{MeOH}(7 \mathrm{~mL})$, saturated aqueous sodium and potassium tartrate $(200 \mathrm{~mL})$, and water $(100 \mathrm{~mL})$ and allowed to warm to $25{ }^{\circ} \mathrm{C}$ over $3 \mathrm{~h}$. EtOAc $(250 \mathrm{~mL})$ was then added, and stirring was continued at $25{ }^{\circ} \mathrm{C}$ for another $30 \mathrm{~min}$. The biphasic mixture was extracted with EtOAc $(4 \times 100 \mathrm{~mL})$ and the combined organic layers were dried $\left(\mathrm{Na}_{2} \mathrm{SO}_{4}\right)$ and concentrated, and the obtained lactol 120 was used in the next step without further purification. To a 
solution of the crude lactol 120 (3.42 g, $7.37 \mathrm{mmol}, 1.0$ equiv) in $\mathrm{CH}_{2} \mathrm{Cl}_{2}(120 \mathrm{~mL})$ at $25^{\circ} \mathrm{C}$ was added tetra-n-butylammonium iodide (5.44 g, $14.7 \mathrm{mmol}, 2.0$ equiv) and $N$-iodosuccinimide (16.6 g, 73.8 mmol, 10 equiv). The resulting dark solution was stirred at $25^{\circ} \mathrm{C}$ for $1 \mathrm{~h}$ and then quenched with saturated aqueous $\mathrm{Na}_{2} \mathrm{~S}_{2} \mathrm{O}_{3}(600 \mathrm{~mL})$. The biphasic mixture was extracted with $\mathrm{CH}_{2} \mathrm{Cl}_{2}(4 \times 100 \mathrm{~mL})$ and the combined organic layers were dried $\left(\mathrm{Na}_{2} \mathrm{SO}_{4}\right)$, concentrated, and then purified by flash column chromatography (silica gel, EtOAc:hexanes 1:1) to afford lactone alcohol 121 (2.28 g, 5.16 mmol, 70\% over two steps) as a colorless oil. 121: $R_{\mathrm{f}}=0.49$ (silica gel, EtOAc:hexanes $\left.1: 1\right) ;[\alpha]_{\mathrm{D}}{ }^{25}=+4.6(c=0.76$, $\mathrm{CHCl}_{3}$ ); IR (film) $v_{\max }=3443,2955,2918,1739,1697,1170 \mathrm{~cm}^{-1} ;{ }^{1} \mathrm{H}$ NMR (500 MHz, $\left.\mathrm{CDCl}_{3}\right): \delta=$ 4.67-4.61 (m, 1 H), 4.30 (ddd, $J=12.1,7.3,4.8$ Hz, 1 H), 4.12 (t, $J=8.4$ Hz, 2 H), 3.75 (dd, $J=9.2,4.8$ Hz, 1 H), 3.75-3.70 (m, 1 H), 3.51 (dd, $J=13.8,7.2$ Hz, 1 H), 2.81 (dd, $J=12.8,11.4$ Hz, 1 H), 2.60 (dd, $J=16.5,4.8 \mathrm{~Hz}, 2 \mathrm{H}), 2.21$ (dd, $J=13.8,7.5 \mathrm{~Hz}, 1 \mathrm{H}), 2.27-2.18$ (m, $1 \mathrm{H}), 2.13$ (dd, $J=16.7,9.4$ Hz, 1 H), 2.02-1.88 (m, 2 H), 1.71 (ddd, $J=14.3,7.3,4.8$ Hz, 1 H), 1.60-1.52 (m, 2 H), 1.23 (q, $J=$ 12.4 Hz, 1 H), 1.09 (d, $J=6.6 \mathrm{~Hz}, 3 \mathrm{H}), 1.02-0.97$ (m, $2 \mathrm{H}), 0.97$ (d, $J=6.6 \mathrm{~Hz}, 3 \mathrm{H}), 0.82$ (d, $J=6.6$ Hz, $3 \mathrm{H}), 0.03$ (s, $9 \mathrm{H}) ;{ }^{13} \mathrm{C}$ NMR (125 $\left.\mathrm{MHz} \mathrm{CDCl}_{3}\right): \delta=171.4$, 155.6, 98.0, 86.5, 78.4, 74.1, 63.3, 49.2, 40.9, 38.2, 37.8, 37.6, 32.5, 30.9, 23.3, 21.2, 18.8, 17.6, 16.9, -1.5; HR-MS [Matrix-assisted laser desorption / ionization (MALDI)]: calcd for $\mathrm{C}_{22} \mathrm{H}_{39} \mathrm{NO}_{6} \mathrm{SiNa}^{+}\left[\mathrm{M}+\mathrm{Na}^{+}\right]$: 464.2439, found 464.2442.

Ketone 122. To a solution of lactone alcohol 121 (940 mg, $2.13 \mathrm{mmol}, 1.0$ equiv) in $\mathrm{CH}_{2} \mathrm{Cl}_{2}(25 \mathrm{~mL})$ at $0{ }^{\circ} \mathrm{C}$ was added pyridine (2.16 mL, $26.7 \mathrm{mmol}, 12$ equiv) and Dess-Martin periodinane (1.36 g, 3.21

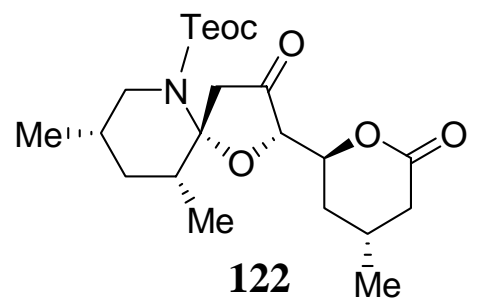
mmol, 1.5 equiv). The reaction mixture was allowed to warm to $25^{\circ} \mathrm{C}$ over a period of $2 \mathrm{~h}$, and then quenched with saturated aqueous $\mathrm{Na}_{2} \mathrm{~S}_{2} \mathrm{O}_{3}$ (40 $\mathrm{mL})$ and saturated aqueous $\mathrm{NaHCO}_{3}(40 \mathrm{~mL})$. The resulting biphasic mixture was then extracted with $\mathrm{CH}_{2} \mathrm{Cl}_{2}(4 \times 20 \mathrm{~mL})$ and the combined organic extracts were dried $\left(\mathrm{Na}_{2} \mathrm{SO}_{4}\right)$, concentrated, and then purified by flash column chromatography (silica gel, EtOAc:hexanes 1:2) to provide ketone 122 (776 mg, $1.77 \mathrm{mmol}$, 83\%) as a colorless solid. 122: $R_{\mathrm{f}}=0.66$ (silica gel, EtOAc:hexanes 1:1); $\mathrm{mp}=72-74{ }^{\circ} \mathrm{C} ;[\alpha]_{\mathrm{D}}{ }^{25}=-55.8\left(c=1.12, \mathrm{CHCl}_{3}\right)$; IR (film) $v_{\max }=2954,2923,1756,1695,1460,1166 \mathrm{~cm}^{-1} ;{ }^{1} \mathrm{H}$ NMR $\left(500 \mathrm{MHz}, \mathrm{CDCl}_{3}\right): \delta=4.64(\mathrm{ddd}, J=$ 
10.3, 4.4, $1.8 \mathrm{~Hz}, 1 \mathrm{H}$ ), 4.31 (d, $J=2.9 \mathrm{~Hz}, 1 \mathrm{H}), 4.14$ (t, $J=8.6 \mathrm{~Hz}, 2 \mathrm{H}), 3.81$ (ddd, $J=13.2,4.4,1.8$ Hz, 1 H), 3.02 (dd, $J=12.8,11.4$ Hz, 1 H), 3.36 (d, $J=19.4$ Hz, 1 H), 2.98 (d, $J=19.4$ Hz, 1 H), 2.57 (dd, $J=15.8,5.1 \mathrm{~Hz}, 1 \mathrm{H}), 2.30-2.17$ (m, 2 H), 2.07 (ddd, $J=16.5$, 9.9, $6.2 \mathrm{~Hz}, 1 \mathrm{H}), 2.02-1.96$ (m, 1 H), 1.79-1.58 (m, 2 H), 1.47-1.35 (m, 2 H), 1.07 (d, $J=6.6$ Hz, 3 H), 1.03-0.97 (m, 2 H), 0.90 (d, $J=$ $6.6 \mathrm{~Hz}, 3 \mathrm{H}), 0.89$ (d, $J=6.2 \mathrm{~Hz}, 3 \mathrm{H}), 0.04$ (s, $9 \mathrm{H}) ;{ }^{13} \mathrm{C}$ NMR $\left(125 \mathrm{MHz}, \mathrm{CDCl}_{3}\right): \delta=210.4,171.4$, 156.5, 94.0, 83.3, 76.9, 63.9, 49.2, 47.0, 40.0, 37.3, 37.2, 30.5, 29.1, 23.8, 20.8, 18.7, 17.7, 16.1, -1.5; HR-MS [Matrix-assisted laser desorption / ionization (MALDI)]: calcd for $\mathrm{C}_{22} \mathrm{H}_{37} \mathrm{NO}_{6} \mathrm{SiNa}^{+}\left[\mathrm{M}+\mathrm{Na}^{+}\right]$: 462.2282, found 462.2268 .

(S)-Alcohol 123. To a solution of ketone 122 (766 mg, $1.74 \mathrm{mmol}, 1.0$ equiv) in THF (20 mL) at $-78{ }^{\circ} \mathrm{C}$ was added lithium tri-sec-butylborohydride (3.13 mL, 1.0 M in THF, 3.13 mmol, 1.8 equiv) over 2 min. Me' The reaction mixture was stirred for $15 \mathrm{~min}$ at $-78{ }^{\circ} \mathrm{C}$ and then quenched with saturated aqueous $\mathrm{NH}_{4} \mathrm{Cl}(80 \mathrm{~mL})$ and water $(80 \mathrm{~mL})$. The biphasic mixture was extracted with $\mathrm{CH}_{2} \mathrm{Cl}_{2}(4 \times 25 \mathrm{~mL})$ and the combined organic layers were dried $\left(\mathrm{Na}_{2} \mathrm{SO}_{4}\right)$, concentrated, and then purified by flash column chromatography (silica gel, EtOAc:hexanes 1:2) to yield a single isomer of (S)-alcohol 123 (609 mg, 1.38 mmol, 79\%) as a colorless oil. 123: $R_{\mathrm{f}}=0.50$ (silica gel, EtOAc:hexanes 1:1); $[\alpha]_{\mathrm{D}}^{25}=+8.7(c$ $\left.=0.85, \mathrm{CHCl}_{3}\right)$; IR (film) $v_{\max }=3432,2951,2921,1738,1695,1459,1173 \mathrm{~cm}^{-1} ;{ }^{1} \mathrm{H} \mathrm{NMR}(500 \mathrm{MHz}$, $\left.\mathrm{CDCl}_{3}\right): \delta=4.76(\mathrm{dt}, J=8.0,4.0 \mathrm{~Hz}, 1 \mathrm{H}), 4.55-4.49(\mathrm{~m}, 1 \mathrm{H}), 4.13(\mathrm{t}, J=8.4 \mathrm{~Hz}, 2 \mathrm{H}), 3.85(\mathrm{dd}, J=$ 8.0, 4.0 Hz, 1 H), 3.81 (d, $J=6.6$ Hz, 1 H), 3.75 (ddd, $J=13.0$, 4.0, $2.0 \mathrm{~Hz}, 1 \mathrm{H}), 3.40$ (dd, $J=15.0,2.0$ Hz, 1 H), 2.94 (dd, $J=13.0,11.0$ Hz, 1 H), 2.63-2.57 (m, 1 H), 2.38 (dd, $J=15.0,6.0$ Hz, 1 H), 2.262.15 (m, 2 H), 2.05-2.00 (m, 1 H), 1.91 (ddd, $J=12.0$, 6.5, 4.0 Hz, 1 H), 1.77-1.73 (m, 1 H), 1.67-1.55 (m, 2 H), 1.26-1.21 (m, 1 H), 1.11 (d, $J=6.5$ Hz, 3 H), 1.02-0.97 (m, 2 H), 0.87 (d, J = 6.5 Hz, 3 H), 0.85 (d, $J=6.5 \mathrm{~Hz}, 3 \mathrm{H}), 0.03$ (s, $9 \mathrm{H}) ;{ }^{13} \mathrm{C}$ NMR (125 MHz, $\left.\mathrm{CDCl}_{3}\right): \delta=171.7,156.6,97.3,85.11$, 74.8, 71.4, 63.7, 49.3, 39.8, 38.4, 38.3, 37.7, 32.3, 30.5, 23.8, 21.4, 18.9, 17.6, 16.9, -1.5; HR-MS [Matrix-assisted laser desorption / ionization (MALDI)]: calcd for $\mathrm{C}_{22} \mathrm{H}_{39} \mathrm{NO}_{6} \mathrm{SiNa}^{+}\left[\mathrm{M}+\mathrm{Na}^{+}\right]$: 464.2439, found 464.2427. 
TES Ether 124. To a solution of (S)-alcohol 123 (608 mg, 1.38 mmol, 1.0 equiv) and 2,6-lutidine (241 $\mu \mathrm{L}, 2.07 \mathrm{mmol}, 1.6$ equiv) in $\mathrm{CH}_{2} \mathrm{Cl}_{2}(15 \mathrm{~mL})$ at $-78{ }^{\circ} \mathrm{C}$ was added TESOTf (343 $\mu \mathrm{L}, 1.52 \mathrm{mmol}, 1.1$

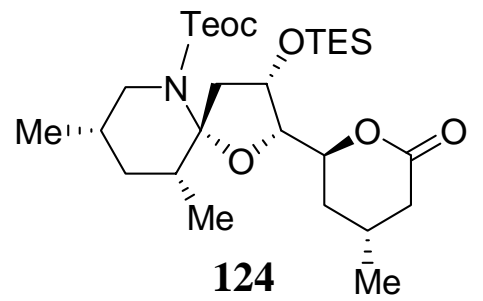
equiv). After 10 min the reaction mixture was quenched with saturated aqueous $\mathrm{NH}_{4} \mathrm{Cl}(50 \mathrm{~mL})$ and water $(50 \mathrm{~mL})$. The biphasic mixture was then extracted with $\mathrm{CH}_{2} \mathrm{Cl}_{2}(4 \times 15 \mathrm{~mL})$ and the combined organic extracts were dried $\left(\mathrm{Na}_{2} \mathrm{SO}_{4}\right)$, concentrated, and then purified by flash column chromatography (silica gel, EtOAc:hexanes 1:3) to give TES ether 124 (717 mg, 1.29 mmol, 93\%) as a colorless oil. 124: $R_{\mathrm{f}}=0.45$ (silica gel, EtOAc:hexanes 1:1); $[\alpha]_{\mathrm{D}}{ }^{25}=-26.5\left(c=0.85, \mathrm{CHCl}_{3}\right)$; IR (film) $v_{\max }=2955,2914,1745,1697,1460,1170 \mathrm{~cm}^{-1} ;{ }^{1} \mathrm{H}$ NMR (500 MHz, $\left.\mathrm{CDCl}_{3}\right): \delta=4.60-4.53(\mathrm{~m}, 2 \mathrm{H})$, 4.20 (dd, $J=7.0,4.5$ Hz, 1 H), 4.18-4.08 (m, 2 H), 3.69 (ddd, $J=13.0,4.0,1.5$ Hz, 1 H), 3.03 (dd, $J=$ 13.0, 12.0 Hz, 1 H), 2.79 (dd, $J=14.5,7.5$ Hz, 1 H), 2.58-2.53 (m, 2 H), 2.28-2.15 (m, 2 H), 2.05 (ddd, $J=16.5,10.5,6.0 \mathrm{~Hz}, 1 \mathrm{H}), 1.91-1.88$ (m, $1 \mathrm{H}), 1.68$ (dt, $J=14.0,4.5 \mathrm{~Hz}, 1 \mathrm{H}), 1.58-1.42$ (m, $2 \mathrm{H})$, 1.29 (q, $J=12.5 \mathrm{~Hz}, 1 \mathrm{H}), 1.09$ (d, $J=6.5 \mathrm{~Hz}, 3 \mathrm{H}), 1.02-0.95$ (m, $2 \mathrm{H}), 0.94$ (t, $J=8.0 \mathrm{~Hz}, 9 \mathrm{H}), 0.80$ (d, $J=6.5 \mathrm{~Hz}, 3 \mathrm{H}), 0.79$ (d, $J=6.5 \mathrm{~Hz}, 3 \mathrm{H}), 0.59$ (q, $J=8.0 \mathrm{~Hz}, 6 \mathrm{H}), 0.04$ (s, $9 \mathrm{H}) ;{ }^{13} \mathrm{C}$ NMR $(125$ $\left.\mathrm{MHz}, \mathrm{CDCl}_{3}\right): \delta=172.3,156.2,96.6,82.8,76.3,71.8,63.1,49.2,43.6,37.9,37.7,37.5,31.1,30.9$, 24.0, 21.0, 18.6, 17.7, 16.7, 6.8, 4.6, -1.5; HR-MS [Matrix-assisted laser desorption / ionization (MALDI)]: calcd for $\mathrm{C}_{28} \mathrm{H}_{53} \mathrm{NO}_{6} \mathrm{Si}_{2} \mathrm{Na}^{+}\left[\mathrm{M}+\mathrm{Na}^{+}\right.$]: 578.3303, found 578.3303.

Ketene Acetal Triflate 125. To a solution of TES ether 125 (25 mg, $45 \mu \mathrm{mol}, 1.0$ equiv) and Comin's reagent 55 (88.4 mg, $225 \mu \mathrm{mol}, 5.0$ equiv) in THF $(0.6 \mathrm{~mL})$ at $-78{ }^{\circ} \mathrm{C}$ was added $\mathrm{KHMDS}(0.405 \mathrm{~mL}$, Me'. $0.5 \mathrm{M}$ in toluene, $203 \mu \mathrm{mol}, 4.0$ equiv). Stirring was continued at $-78{ }^{\circ} \mathrm{C}$ for $45 \mathrm{~min}$ and then the reaction was quenched with saturated aqueous $\mathrm{NaHCO}_{3}(10 \mathrm{~mL})$. The biphasic mixture was extracted with $\mathrm{CH}_{2} \mathrm{Cl}_{2}(4 \times$ $10 \mathrm{~mL})$ and the combined organic layers were dried $\left(\mathrm{Na}_{2} \mathrm{SO}_{4}\right)$, concentrated, and then purified by flash column chromatography (silica gel, EtOAc:hexanes:Et ${ }_{3} \mathrm{~N}$ 1:100:0.5) to afford ketene acetal triflate $125(27.6 \mathrm{mg}, 40.1 \mu \mathrm{mol}, 89 \%)$ as a colorless oil. 125: $R_{\mathrm{f}}=$ 
0.80 (silica gel, EtOAc:hexanes 1:4); $[\alpha]_{\mathrm{D}}{ }^{25}=+4.5\left(c=0.84, \mathrm{CHCl}_{3}\right)$; IR (film) $v_{\max }=2957,2917$, 1697, 1427, $1141 \mathrm{~cm}^{-1} ;{ }^{1} \mathrm{H}$ NMR (500 MHz, $\mathrm{C}_{6} \mathrm{D}_{6}$ ): $\delta=4.54$ (ddd, $J=9.0,5.5,2.5 \mathrm{~Hz}, 1 \mathrm{H}$ ), 4.49 (q, $J$ = $6.5 \mathrm{~Hz}, 1 \mathrm{H}), 4.44$ (d, $J=4.0 \mathrm{~Hz}, 1 \mathrm{H}), 4.29-4.24(\mathrm{~m}, 2 \mathrm{H}), 4.16$ (t, $J=6.0 \mathrm{~Hz}, 1 \mathrm{H}), 3.97$ (dd, $J=$ 13.0, $4.0 \mathrm{~Hz}, 1 \mathrm{H}$ ), 3.37 (dd, $J=$ 14.7, $6.2 \mathrm{~Hz}, 1 \mathrm{H}$ ), 3.24 (dd, $J=13.0,12.0 \mathrm{~Hz}, 1 \mathrm{H}$ ), 2.56 (dd, $J=14.5$, 6.5 Hz, 1 H), 2.18-1.96 (m, 3 H), 1.65 (dt, $J=14.0,3.0$ Hz, 1 H), 1.63-1.59 (m, 1 H), 1.39-1.29 (m, 2 H), 1.00 (t, $J=8.0 \mathrm{~Hz}, 9 \mathrm{H}), 1.05-0.90$ (m, $2 \mathrm{H}), 0.80$ (d, $J=7.0 \mathrm{~Hz}, 3 \mathrm{H}), 0.75$ (d, $J=6.5 \mathrm{~Hz}, 3 \mathrm{H})$, 0.73 (d, $J=6.5 \mathrm{~Hz}, 3 \mathrm{H}), 0.62$ (q, $J=8.0 \mathrm{~Hz}, 6 \mathrm{H}),-0.07$ (s, $9 \mathrm{H}) ;{ }^{13} \mathrm{C}$ NMR $\left(125 \mathrm{MHz}, \mathrm{C}_{6} \mathrm{D}_{6}\right): \delta=$ 156.3, 149.9, 119.3, 97.2, 93.9, 82.4, 76.3, 72.4, 63.2, 49.5, 43.2, 38.6, 37.7, 31.6, 30.9, 25.0, 21.9, 18.8, 18.0, 16.9, 7.05, 4.95, -0.07; HR-MS [Matrix-assisted laser desorption / ionization (MALDI)]: calcd for $\mathrm{C}_{29} \mathrm{H}_{52} \mathrm{~F}_{3} \mathrm{NO}_{8} \mathrm{SSi}_{2} \mathrm{Na}^{+}\left[\mathrm{M}+\mathrm{Na}^{+}\right]:$710.2796, found 710.2814.

Stannane 98. To a solution of ketene acetal triflate 125 (300 mg, 0.44 mmol, 1.0 equiv) in THF (15 mL) at $25{ }^{\circ} \mathrm{C}$ was added tri-2-furylphosphine ( $51 \mathrm{mg}, 0.22 \mathrm{mmol}, 0.5$ equiv), $\mathrm{LiCl}$ ( $55 \mathrm{mg}, 1.32 \mathrm{mmol}, 3.0$ Me'. equiv), a solution of $\mathrm{Sn}_{2} \mathrm{Me}_{6}$ (0.8 g, $2.2 \mathrm{mmol}, 5.0$ equiv) in THF (0.5 $\mathrm{mL}$ ), and $\mathrm{Pd}_{2} \mathrm{dba}_{3}$ (40 mg, $0.044 \mathrm{mmol}, 0.1$ equiv). The reaction mixture was allowed to stir for $1 \mathrm{~h}$, and was then quenched with saturated aqueous $\mathrm{NaHCO}_{3}(20 \mathrm{~mL})$. The biphasic mixture was extracted with EtOAc $(3 \times 15 \mathrm{~mL})$ and the combined organic extracts were dried $\left(\mathrm{Na}_{2} \mathrm{SO}_{4}\right)$, concentrated, and then purified by flash column chromatography (silica gel, EtOAc:hexanes: $\mathrm{Et}_{3} \mathrm{~N}$ 1:100:0.5) to afford stannane 98 (303 mg, $0.43 \mathrm{mmol}, 98 \%$ ) as a colorless oil. 98: $R_{\mathrm{f}}=0.53$ (silica gel, EtOAc:hexanes 1:5); $[\alpha]_{\mathrm{D}}^{25}=-24.9\left(c=0.99, \mathrm{CHCl}_{3}\right)$; IR (film) $v_{\max }=2952,2913,2874,1698,1466$, 1392, 1249, $1166 \mathrm{~cm}^{-1}$; ${ }^{1} \mathrm{H}$ NMR (500 MHz, $\left.\mathrm{C}_{6} \mathrm{D}_{6}\right): \delta=5.09$ (d, $\left.J=4.0 \mathrm{~Hz}, 1 \mathrm{H}\right), 4.72(\mathrm{q}, J=10.5,5.5$ Hz, 1 H), 4.66 (ddd, $J=9.0,6.5,3.0 \mathrm{~Hz}, 1 \mathrm{H}), 4.46$ (t, $J=8.5 \mathrm{~Hz}, 2 \mathrm{H}), 4.42$ (t, $J=6.0 \mathrm{~Hz}, 1 \mathrm{H}$ ), 4.15 (dd, $J=14.5,3.0 \mathrm{~Hz}, 1 \mathrm{H}$ ), 3.81 (dd, $J=14.5,5.0 \mathrm{~Hz}, 1 \mathrm{H}$ ), 3.53 (dd, $J=13.0,12.0 \mathrm{~Hz}, 1 \mathrm{H}$ ), 2.65 (dd, $J$ = 15.0, $6.0 \mathrm{~Hz}, 1 \mathrm{H}), 2.54-2.50$ (m, $1 \mathrm{H}), 2.44-2.30$ (m, $1 \mathrm{H}), 2.29-2.25$ (m, $1 \mathrm{H}), 2.10-2.05(\mathrm{~m}, 1 \mathrm{H})$, 1.83-1.75 (m, 1 H), 1.27 (d, $J=7.0$ Hz, 3 H), 1.23 (t, $J=8.0$ Hz, 9 H), 0.95 (d, $J=7.0$ Hz, 3 H), 0.93 (d, $J=7.0 \mathrm{~Hz}, 3 \mathrm{H}), 0.85$ (q, $J=8.0 \mathrm{~Hz}, 6 \mathrm{H}), 0.41(\mathrm{~s}, 9 \mathrm{H}), 0.11(\mathrm{~s}, 9 \mathrm{H}) ;{ }^{13} \mathrm{C}$ NMR $\left(125 \mathrm{MHz}, \mathrm{C}_{6} \mathrm{D}_{6}\right): \delta=$ 
156.5, 118.7, 97.2, 84.3, 72.8, 71.5, 63.1, 49.6, 42.6, 39.2, 37.9, 32.6, 31.9, 25.5, 23.2, 19.1, 18.2, 17.2, 16.5, 7.4, 5.4, -9.4; HR-MS [Matrix-assisted laser desorption / ionization (MALDI)]: calcd for $\mathrm{C}_{31} \mathrm{H}_{61} \mathrm{NO}_{5} \mathrm{Si}_{2} \mathrm{SnNa}^{+}\left[\mathrm{M}+\mathrm{Na}^{+}\right]:$704.3183, found 704.3171.

Allylated Product 126. To a solution of ketene acetal triflate 125 (218 mg, $317 \mu \mathrm{mol}, 1.0$ equiv) in THF ( $4 \mathrm{~mL}$ ) at $25^{\circ} \mathrm{C}$ was added lithium chloride (40.3 mg, $951 \mu \mathrm{mol}, 3.0$ equiv), $\mathrm{Pd}_{2} \mathrm{dba}_{3} \cdot \mathrm{CHCl}_{3}(16.4$

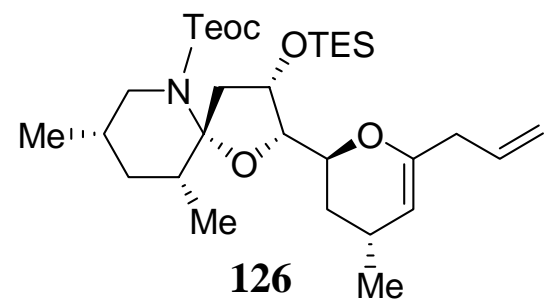
mg, $15.8 \mu \mathrm{mol}$, 0.05 equiv), and tri-2-furylphosphine (31.6 mg, 136 $\mu$ mol, 0.4 equiv). After stirring for $10 \mathrm{~min}$, allyl-tri- $n$-butyltin (547 mg, 1.58 mmol, 5.0 equiv) was added, and the mixture was stirred at $25{ }^{\circ} \mathrm{C}$ for $15 \mathrm{~h}$. The reaction was then quenched with saturated aqueous $\mathrm{NaHCO}_{3}(50 \mathrm{~mL})$, and the resulting biphasic mixture was extracted with $\mathrm{Et}_{2} \mathrm{O}(4 \times 20 \mathrm{~mL})$. The combined organic extracts were dried $\left(\mathrm{Na}_{2} \mathrm{SO}_{4}\right)$, concentrated, and then purified by flash column chromatography (silica gel, EtOAc:hexanes 1:9) to afford allylated product 126 (161 mg, $271 \mu$ mol, 85\%) as a colorless oil. 126: $R_{\mathrm{f}}=0.24$ (silica gel, hexanes:Et $\left.2 \mathrm{O} 19: 1\right) ;[\alpha]_{\mathrm{D}}{ }^{25}=+17.2\left(c=0.39, \mathrm{CHCl}_{3}\right)$; IR (film) $v_{\max }=2954,2908,2872,1697,1459,1254,1171 \mathrm{~cm}^{-1} ;{ }^{1} \mathrm{H}$ NMR $\left(500 \mathrm{MHz}, \mathrm{CDCl}_{3}\right): \delta=$ 5.85-5.81 (m, 1 H), 5.06 (d, $J=17.0$ Hz, 1 H), 5.01 (d, $J=10.0$ Hz, 1 H), 4.51-4.47 (m, 1 H), 4.45 (br s, $1 \mathrm{H}), 4.12-4.08$ (m, $2 \mathrm{H}), 4.05-4.03(\mathrm{~m}, 1 \mathrm{H}), 3.68$ (d, $J=12.0 \mathrm{~Hz}, 1 \mathrm{H}), 3.07$ (t, $J=12.0 \mathrm{~Hz}, 1 \mathrm{H})$, 2.99 (dd, $J=14.5,6.1 \mathrm{~Hz}, 1 \mathrm{H}$ ), 2.74 (d, $J=6.1 \mathrm{~Hz}, 2 \mathrm{H}), 2.42$ (dd, $J=14.5,6.6 \mathrm{~Hz}, 1 \mathrm{H}$ ), 2.24 (br s, 1 H), 1.93-1.87 (m, 1 H), 1.85-1.79 (m, 1 H), 1.63-1.51 (m, 2 H), 1.47-1.43 (m, 1 H), 1.34-1.28 (m, 1 H), 0.98 (d, $J=6.5 \mathrm{~Hz}, 3 \mathrm{H}), 0.96$ (d, $J=6.5 \mathrm{~Hz}, 3 \mathrm{H}), 0.93$ (t, $J=8.0 \mathrm{~Hz}, 9 \mathrm{H}), 0.79$ (d, $J=6.5 \mathrm{~Hz}, 6$ H), 0.58 (q, $J=8.0 \mathrm{~Hz}, 6 \mathrm{H}), 0.03$ (s, $9 \mathrm{H}) ;{ }^{13} \mathrm{C}$ NMR (125 MHz, $\left.\mathrm{CDCl}_{3}\right): \delta=157.2,152.6,136.0$, 117.1, 103.3, 97.2, 83.9, 73.0, 71.8, 63.8, 50.0, 43.9, 39.6, 39.3, 38.6, 32.2, 32.1, 25.3, 23.7, 19.6, 18.8, 17.7, 7.8, 5.7, -1.2; HR-MS [Matrix-assisted laser desorption / ionization (MALDI)]: calcd for $\mathrm{C}_{31} \mathrm{H}_{57} \mathrm{NO}_{5} \mathrm{Si}_{2} \mathrm{H}^{+}\left[\mathrm{M}+\mathrm{H}^{+}\right]$: 580.3848, found 580.3848 . 
Alcohol 127. Allylated product 126 (161 mg, $271 \mu \mathrm{mol}, 1.0$ equiv) was dissolved in a mixture of pyridine:THF (1:1, $4 \mathrm{ml})$ and cooled to $0{ }^{\circ} \mathrm{C}$. HF•pyridine $(0.4 \mathrm{ml})$ was added dropwise and the reaction

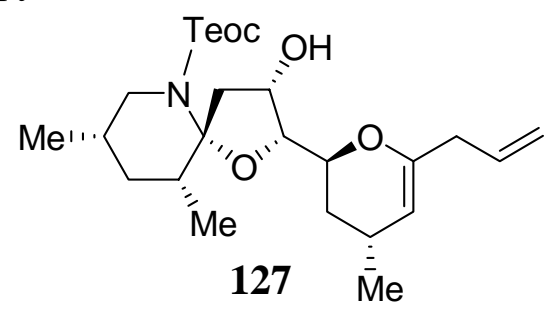
mixture was stirred at $0{ }^{\circ} \mathrm{C}$ for $5 \mathrm{~h}$ and then quenched by the slow addition of saturated aqueous $\mathrm{NaHCO}_{3}(10 \mathrm{ml})$. The biphasic mixture was extracted with EtOAc $(3 \times 7 \mathrm{ml})$, and the combined organic extracts were dried $\left(\mathrm{Na}_{2} \mathrm{SO}_{4}\right)$, concentrated, and then purified by flash column chromatography (silica gel, hexanes:EtOAc 4:1) to afford alcohol 127 (116 mg, 255 mol, 94\%) as a colorless oil. 127: $R_{\mathrm{f}}=0.28$ (silica gel, EtOAc:hexanes 1:6); $[\alpha]_{\mathrm{D}}{ }^{25}=+21.8\left(c=0.62, \mathrm{CHCl}_{3}\right)$; IR (film) $v_{\max }=3460,2955,2907,2872,1697,1672,1386,1173 \mathrm{~cm}^{-1}$; ${ }^{1} \mathrm{H}$ NMR $\left(500 \mathrm{MHz}, \mathrm{CDCl}_{3}\right): \delta=$ 5.80 (tq, $J=10.5,6.5 \mathrm{~Hz}, 1 \mathrm{H}$ ), 5.07 (dd, $J=17.0,1.5 \mathrm{~Hz}, 1 \mathrm{H}$ ), 5.03 (dd, $J=10.5,1.5 \mathrm{~Hz}, 1 \mathrm{H}$ ), 4.52 (d, $J=4.0 \mathrm{~Hz}, 1 \mathrm{H}$ ), 4.48-4.46 (m, 1 H), 4.21 (dt, $J=8.5,2.5 \mathrm{~Hz}, 1 \mathrm{H}$ ), 4.13 (t, $J=8.5 \mathrm{~Hz}, 2 \mathrm{H}$ ), 3.88 (dd, $J$ = 9.0, $5.0 \mathrm{~Hz}, 1 \mathrm{H}$ ), 3.72 (ddd, $J$ = 13.5, 4.0, $2.0 \mathrm{~Hz}, 1 \mathrm{H}$ ), 3.37-3.32 (m, $2 \mathrm{H}$ ), 3.02 (dd, $J$ = 13.5, $8.5 \mathrm{~Hz}, 1 \mathrm{H}$ ), 2.74 (d, $J=6.5 \mathrm{~Hz}, 2 \mathrm{H}$ ), 2.40 (dd, $J=15.0,6.0 \mathrm{~Hz}, 1 \mathrm{H}), 2.30-2.26$ (m, $1 \mathrm{H}$ ), 1.97-1.92 (m, 1 H), 1.88-1.82 (m, 1 H), 1.71-1.67 (m, 2 H), 1.62-1.56 (m, 1 H), 1.55-1.51 (m, 1 H), 1.26 (q, $J=$ $12.5 \mathrm{~Hz}, 2 \mathrm{H}$ ), 1.02 (d, $J=6.5 \mathrm{~Hz}, 3 \mathrm{H}), 0.85$ (d, $J=6.5 \mathrm{~Hz}, 3 \mathrm{H}$ ), 0.83 (d, $J=6.5 \mathrm{~Hz}, 3 \mathrm{H}$ ), 0.03 (s, 9 $\mathrm{H}) ;{ }^{13} \mathrm{C}$ NMR $\left(125 \mathrm{MHz}, \mathrm{CDCl}_{3}\right): \delta=156.6,150.6,134.8,116.7,103.1,97.1,82.6,72.5,70.8,63.3$, 49.3, 40.3, 38.5, 38.4, 37.4, 32.2, 30.9, 23.9, 22.4, 18.8, 17.6, 16.7, -1.5; HR-MS [Matrix-assisted laser desorption / ionization (MALDI)]: calcd for $\mathrm{C}_{25} \mathrm{H}_{43} \mathrm{NO}_{5} \mathrm{SiNa}^{+}\left[\mathrm{M}+\mathrm{Na}^{+}\right]$: 488.2803, found 488.2799.

Tetracyclic Iodide 129. To a solution of alcohol 127 (30 mg, $0.05 \mathrm{mmol}, 1.0$ equiv) in THF (5 mL) at 0 ${ }^{\circ} \mathrm{C}$ was added $\mathrm{NaHCO}_{3}(125 \mathrm{mg}, 1.50 \mathrm{mmol}, 30$ equiv) and $\mathrm{N}$-iodosuccinimide (113 mg, $0.50 \mathrm{mmol}, 10$

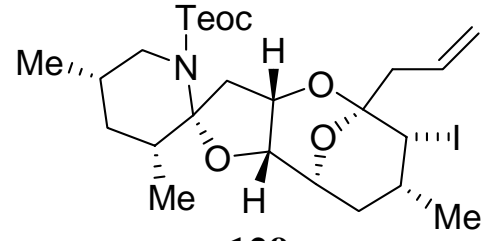

129 equiv). The reaction mixture was stirred at $0{ }^{\circ} \mathrm{C}$ for $12 \mathrm{~h}$, then quenched with saturated aqueous $\mathrm{Na}_{2} \mathrm{~S}_{2} \mathrm{O}_{3}(10 \mathrm{~mL})$. The resulting biphasic mixture was extracted with EtOAc $(3 \times 10 \mathrm{~mL})$, and the combined organic extracts were dried $\left(\mathrm{Na}_{2} \mathrm{SO}_{4}\right)$, concentrated, and then purified by flash column chromatography (silica gel, EtOAc:hexanes 1:1) to provide a single isomer of tetracyclic iodide 129 (25 mg, $0.039 \mathrm{mmol}, 77 \%)$ as a colorless oil. The sample was then crystallized from a 1:1 solution of 
$\mathrm{Et}_{2} \mathrm{O}$ :pentane $(1 \mathrm{~mL})$ to obtain colorless crystals to be used for X-ray analysis. 129: $R_{\mathrm{f}}=0.40$ (silica gel, EtOAc:hexanes 1:6); $\mathrm{mp}=130-136^{\circ} \mathrm{C} ;[\alpha]_{\mathrm{D}}{ }^{25}=+12.1\left(c=0.85, \mathrm{CHCl}_{3}\right) ; \mathrm{IR}(\mathrm{film}) v_{\max }=2955,1695$, 1459, 1351, 1255, $1172 \mathrm{~cm}^{-1}$; ${ }^{1} \mathrm{H}$ NMR (600 MHz, $\left.\mathrm{CDCl}_{3}\right): \delta=5.76-5.69$ (m, $\left.1 \mathrm{H}\right), 5.24$ (d, $J=17.0$ Hz, $1 \mathrm{H}), 5.13$ (d, $J=10.5 \mathrm{~Hz}, 1 \mathrm{H}), 4.74-4.72$ (m, $1 \mathrm{H}), 4.25$ (d, $J=5.3 \mathrm{~Hz}, 1 \mathrm{H}), 4.12-4.04$ (m, $3 \mathrm{H})$, 3.86 (d, $J=6.2 \mathrm{~Hz}, 1 \mathrm{H}$ ), 3.74-3.71 (m, 2 H), 3.18 (t, $J=12.2 \mathrm{~Hz}, 1 \mathrm{H}), 2.98$ (dd, $J=14.0,9.2 \mathrm{~Hz}, 1 \mathrm{H}$ ), 2.49 (dd, $J=14.0,6.0 \mathrm{~Hz}, 1 \mathrm{H}$ ), 2.27 (dd, $J=14.5,6.0 \mathrm{~Hz}, 1 \mathrm{H}), 2.03-1.97$ (m, $1 \mathrm{H}), 1.90-1.84$ (m, 1 H), 1.57-1.50 (m, 2 H), 1.34-1.31 (m, 2 H), 1.29-1.22 (m, 1 H), 0.95 (d, J = 6.6 Hz, 3 H), 0.96-0.92 (m, $2 \mathrm{H}), 0.79$ (d, $J=6.6 \mathrm{~Hz}, 3 \mathrm{H}), 0.78$ (d, $J=6.6 \mathrm{~Hz}, 3 \mathrm{H}), 0.02$ (s, $9 \mathrm{H}) ;{ }^{13} \mathrm{C}$ NMR (125 MHz, $\left.\mathrm{CDCl}_{3}\right): \delta$ $=157.1,132.0,120.5,98.1,97.9,79.6,75.0,72.9$, 63.8, 50.0, 49.8, 47.4, 41.0, 39.7, 37.5, 32.2, 31.8, 29.2, 25.7, 19.6, 18.8, 17.3, -0.5; HR-MS [Matrix-assisted laser desorption / ionization (MALDI)]: calcd for $\mathrm{C}_{25} \mathrm{H}_{42} \mathrm{INO}_{5} \mathrm{SiNa}^{+}\left[\mathrm{M}+\mathrm{Na}^{+}\right]$: 614.1769, found 614.1785.

Des-iodo product 130. To a solution of tetracyclic iodide 129 (25 mg, 0.039 mmol, 1.0 equiv) in $n$ $\mathrm{Bu}_{3} \mathrm{SnH}$ :toluene $(1: 2,2 \mathrm{~mL})$ at $0{ }^{\circ} \mathrm{C}$ was added $\mathrm{Et}_{3} \mathrm{~B}(0.12 \mathrm{~mL}, 1.0 \mathrm{M}$ in hexanes, $0.117 \mathrm{mmol}, 3.0$

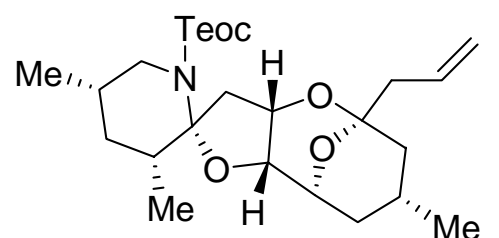

130 equiv), and the reaction mixture was stirred for $5 \mathrm{~min}$. When complete the reaction purified directly by flash column chromatography (silica gel, EtOAc:hexanes 1:5) to afford des-iodo product 130 (17.1 mg, $0.037 \mathrm{mmol}$, 94\%) as a colorless oil. 130: $R_{\mathrm{f}}=0.39$ (silica gel, EtOAc:hexanes 1:6); $[\alpha]_{\mathrm{D}}{ }^{25}=+2.4\left(c=0.58, \mathrm{CHCl}_{3}\right)$; IR (film) $v_{\max }=2953,1695,1459,1394,1254,1171 \mathrm{~cm}^{-1} ;{ }^{1} \mathrm{H}$ NMR (500 MHz, $\left.\mathrm{CDCl}_{3}\right): \delta=5.91-5.85$ (m, $\left.1 \mathrm{H}\right), 5.10-5.06(\mathrm{~m}, 2 \mathrm{H}), 4.83-4.81(\mathrm{~m}, 1 \mathrm{H}), 4.40(\mathrm{~d}, J=4.5$ Hz, 1 H), 4.22-4.13 (m, 2 H), 3.97 (d, $J=9.0$ Hz, 1 H), 3.85-3.81 (m, 2 H), 3.29 (t, J = $12.0 \mathrm{~Hz}, 1 \mathrm{H})$, 2.32 (d, $J=7.0$ Hz, 2 H), 2.23-2.15 (m, 2 H), 2.12-2.07 (m, 1 H), 1.93-1.88 (m, 1 H), 1.76-1.72 (m, 1 H), 1.68-1.59 (m, 2 H), 1.58-1.50 (m, 1 H), 1.42-1.34 (m, 1 H), 1.32-1.27 (m, 1 H), 1.06-1.01 (m, 2 H), 0.96 (d, $J=6.5 \mathrm{~Hz}, 3 \mathrm{H}), 0.89$ (d, $J=6.5 \mathrm{~Hz}, 3 \mathrm{H}), 0.87$ (d, $J=6.5 \mathrm{~Hz}, 3 \mathrm{H}), 0.08(\mathrm{~s}, 9 \mathrm{H}) ;{ }^{13} \mathrm{C}$ NMR $\left(125 \mathrm{MHz}, \mathrm{CDCl}_{3}\right): \delta=156.3,133.9,117.5,97.5,96.4,78.6,74.1,71.6,63.1,48.8,47.1,42.5,39.5$, 36.9, 35.3, 32.9, 31.8, 25.8, 23.5, 19.2, 18.2, 16.9, -0.9; HR-MS [Matrix-assisted laser desorption / ionization (MALDI)]: calcd for $\mathrm{C}_{25} \mathrm{H}_{43} \mathrm{NO}_{5} \mathrm{SiNa}^{+}\left[\mathrm{M}+\mathrm{Na}^{+}\right]$: 488.2808, found 488.2842. 
Dithiane 132. To a solution of lactol 131 (1.83 g, $14.1 \mathrm{mmol}, 1.0$ equiv) and 1,3-propanedithiol (1.56 mL, 15.5 mmol, 1.1 equiv) in $\mathrm{CH}_{2} \mathrm{Cl}_{2}(80 \mathrm{~mL})$ at $0{ }^{\circ} \mathrm{C}$ was added $\mathrm{BF}_{3} \cdot \mathrm{OEt}_{2}(2.68 \mathrm{~mL}, 21.1 \mathrm{mmol}, 1.5$<smiles>CC(C[C@@H](C)CO)C1SCCCS1</smiles>

132 equiv) dropwise over $10 \mathrm{~min}$. Stirring was continued at $0{ }^{\circ} \mathrm{C}$ for $45 \mathrm{~min}$, and then the reaction was quenched with saturated aqueous $\mathrm{NaHCO}_{3}(150 \mathrm{~mL})$. The biphasic mixture was stirred for $30 \mathrm{~min}$ at $25^{\circ} \mathrm{C}$ and then extracted with $\mathrm{CH}_{2} \mathrm{Cl}_{2}(4 \times 50 \mathrm{~mL})$.

The combined organic extracts were dried $\left(\mathrm{Na}_{2} \mathrm{SO}_{4}\right)$, concentrated, and then purified by flash column chromatography (silica gel, EtOAc:hexanes 1:4 $\rightarrow$ 1:2) to give dithiane 132 (2.85 g, 12.9 mmol, 91\%) as a colorless oil. 132: $R_{\mathrm{f}}=0.17$ (silica gel, EtOAc:hexanes 1:4); $[\alpha]_{\mathrm{D}}{ }^{25}=+8.5\left(\mathrm{CHCl}_{3}, c=0.80\right)$; IR (film) $v_{\max }=3394,2954,2872,1457,1275,1185 \mathrm{~cm}^{-1} ;{ }^{1} \mathrm{H}$ NMR $\left(500 \mathrm{MHz}, \mathrm{CDCl}_{3}\right): \delta=4.15(\mathrm{~d}, J=$ $4.0 \mathrm{~Hz}, 1 \mathrm{H}$ ), 3.53 (dd, $J=10.5,5.0 \mathrm{~Hz}, 1 \mathrm{H}), 3.43$ (dd, $J=10.5,6.0 \mathrm{~Hz}, 1 \mathrm{H}), 2.92-2.82$ (m, $4 \mathrm{H})$, 2.15-2.08 (m, 1 H), 2.06-2.00 (m, 1 H), 1.89-1.68 (m, 3 H), 1.40 (s, 1 H), 1.10 (d, J = 6.5 Hz, 3 H), 1.16-1.05 (m, $1 \mathrm{H}), 0.96$ (d, $J=6.5 \mathrm{~Hz}, 3 \mathrm{H}) ;{ }^{13} \mathrm{C}$ NMR (125 MHz, $\left.\mathrm{CDCl}_{3}\right): \delta=67.9,55.1,37.7,36.0$, 33.3, 31.2, 30.8, 26.4, 17.7, 17.4; HR-MS [Matrix-assisted laser desorption / ionization (MALDI)]: calcd for $\mathrm{C}_{10} \mathrm{H}_{20} \mathrm{OS}_{2} \mathrm{Na}^{+}\left[\mathrm{M}+\mathrm{Na}^{+}\right]$: 221.1028, found 221.1036.

Aldehyde 12. To a solution of oxalyl chloride (1.35 mL, $15.4 \mathrm{mmol}, 1.2$ equiv) in $\mathrm{CH}_{2} \mathrm{Cl}_{2}(40 \mathrm{~mL})$ at $78^{\circ} \mathrm{C}$ was added DMSO (2.20 mL, $31.0 \mathrm{mmol}, 2.4$ equiv). After $15 \mathrm{~min}$, a solution of dithiane 132 (2.85<smiles>[Y12][C@@H](C)C[C@H](C)C=O</smiles>

12

g, 12.9 mmol, 1.0 equiv) in $\mathrm{CH}_{2} \mathrm{Cl}_{2}(40 \mathrm{~mL})$ was added dropwise. The reaction mixture was stirred at $-78{ }^{\circ} \mathrm{C}$ for $40 \mathrm{~min}$ and then triethylamine $(8.99 \mathrm{~mL}, 64.5$ mmol, 4.8 equiv) was added and the reaction mixture was stirred at $0{ }^{\circ} \mathrm{C}$ for $1 \mathrm{~h}$.

The reaction was then quenched with saturated aqueous $\mathrm{NaCl}(200 \mathrm{~mL})$ and extracted with $\mathrm{CH}_{2} \mathrm{Cl}_{2}(4 \times$ $50 \mathrm{~mL})$. The combined organic extracts were dried $\left(\mathrm{Na}_{2} \mathrm{SO}_{4}\right)$, concentrated and then purified by flash column chromatography (silica gel, EtOAc:hexanes 1:4) to afford aldehyde 12 (2.32 g, 10.6 mmol, 82\%) as a colorless oil. 12: $R_{\mathrm{f}}=0.47$ (silica gel, EtOAc:hexanes 1:4); $[\alpha]_{\mathrm{D}}{ }^{25}=+12.3\left(\mathrm{CHCl}_{3}, c=0.80\right)$; IR (film) $v_{\max }=2963,2931,2820,1723,1458,1186 \mathrm{~cm}^{-1} ;{ }^{1} \mathrm{H}$ NMR $\left(500 \mathrm{MHz}, \mathrm{CDCl}_{3}\right): \delta=9.58(\mathrm{~d}, J$ $=2.5 \mathrm{~Hz}, 1 \mathrm{H}), 4.12$ (d, $J=4.0 \mathrm{~Hz}, 1 \mathrm{H}), 2.95-2.83$ (m, $4 \mathrm{H}), 2.50-2.43$ (m, $1 \mathrm{H}), 2.15-2.05(\mathrm{~m}, 2 \mathrm{H})$, 
2.03-1.94 (m, 1 H), 1.89-1.76 (m, 1 H), 1.34 (ddd, $J=14.5,8.5,6.0$ Hz, 1 H), 1.12 (d, $J$ = 7.0 Hz, 3 H), 1.11 (d, $J=7.0 \mathrm{~Hz}, 3 \mathrm{H})$; ${ }^{13} \mathrm{C}$ NMR (125 $\left.\mathrm{MHz} \mathrm{CDCl}_{3}\right): \delta=204.7,54.9,44.2,36.1,35.1,31.1,30.7$, 26.3, 17.3, 14.2; HR-MS [Matrix-assisted laser desorption / ionization (MALDI)]: calcd for $\mathrm{C}_{10} \mathrm{H}_{16} \mathrm{~S}_{2} \mathrm{Na}^{+}\left[\mathrm{M}+\mathrm{Na}^{+}-\mathrm{H}_{2} \mathrm{O}\right]:$ 201.0772, found 201.0776.

NHK Coupling Product 135. A solution of aldehyde 12 (220 mg, 1.09 mmol, 1.0 equiv) and vinyl iodide 104 (0.816 g, $2.74 \mathrm{mmol}, 2.5$ equiv) in $\mathrm{DMF}$ (3 mL) was added to a solution of $\mathrm{NiCl}_{2}(2.8 \mathrm{mg}$,<smiles>C=C(COS(=O)(=O)c1ccccc1)[C@H](O)C(C)C[C@H](C)C1SCCCS1</smiles>

135

$0.02 \mathrm{mmol}, 0.02$ equiv) and $\mathrm{CrCl}_{2}$ (0.6572 g, $4.36 \mathrm{mmol}, 4.0$ equiv) in $\mathrm{DMF}$ $(3 \mathrm{~mL})$ at $0{ }^{\circ} \mathrm{C}$. The reaction mixture was then allowed to warm to $25{ }^{\circ} \mathrm{C}$ over $15 \mathrm{~h}$ and then quenched with saturated aqueous $\mathrm{NaHCO}_{3}(10 \mathrm{~mL})$. The resulting biphasic mixture was extracted with $\mathrm{Et}_{2} \mathrm{O}(3 \times 10 \mathrm{~mL})$ and the combined organic layers were dried $\left(\mathrm{Na}_{2} \mathrm{SO}_{4}\right)$, concentrated, and then purified by flash column chromatography (silica gel, EtOAc:hexanes 1:1) to afford a 3:2 ratio of NHK coupling products 135 (404 mg, 1.04 mmol, 95\%) as a colorless oil. 135 (mixture of epimers ca 3:2, major epimer listed): $R_{\mathrm{f}}=0.46$ (silica gel, EtOAc:hexanes 1:4); IR (film) $v_{\max }=3463,2929,2856,1422,1079 \mathrm{~cm}^{-1} ;{ }^{1} \mathrm{H} \mathrm{NMR}\left(500 \mathrm{MHz}, \mathrm{CDCl}_{3}\right)$ : $\delta=5.16-5.12(\mathrm{~m}, 1 \mathrm{H}), 5.06(\mathrm{~s}, 1 \mathrm{H}), 4.38-4.16(\mathrm{~m}, 3 \mathrm{H}), 3.93(\mathrm{t}, J=6.0 \mathrm{~Hz}, 1 \mathrm{H}), 2.98-2.90(\mathrm{~m}, 1 \mathrm{H})$, 2.89-2.80 (m, 3 H), 2.43 (d, $J=5.5$ Hz, 1 H), 2.15-1.63 (m, 5 H), 1.11 (d, $J=7.0$ Hz, 3 H), 1.08-1.01 (m, $1 \mathrm{H}), 0.92$ (s, $9 \mathrm{H}), 0.87$ (d, $J=6.5 \mathrm{~Hz}, 3 \mathrm{H}), 0.09$ (s, $3 \mathrm{H}), 0.08$ (s, $3 \mathrm{H}) ;{ }^{13} \mathrm{C}$ NMR $(125 \mathrm{MHz}$, $\left.\mathrm{CDCl}_{3}\right): \delta=148.4,112.7,77.7,64.9,54.5,37.0,35.7,34.5,31.3,30.7,26.5,25.9,18.3,17.9,14.6,-$ 5.42, -5.45; HR-MS [Matrix-assisted laser desorption / ionization (MALDI)]: calcd for $\mathrm{C}_{19} \mathrm{H}_{38} \mathrm{O}_{2} \mathrm{~S}_{2} \mathrm{SiNa}^{+}\left[\mathrm{M}+\mathrm{Na}^{+}\right]:$413.1975, found 413.1991. 
Enone 136. IBX (0.87 g, $3.08 \mathrm{mmol}, 2.0$ equiv) was dissolved in DMSO (3 mL) and heated to $50{ }^{\circ} \mathrm{C}$ until the mixture became clear, at which time the solution was cooled to $25^{\circ} \mathrm{C}$ and added dropwise to a

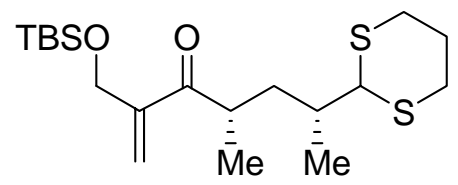

136 solution of NHK coupling partner 135 (0.581 g, 1.54 mmol, 1.0 equiv) in THF $(0.7 \mathrm{~mL})$ cooled to $0^{\circ} \mathrm{C}$. The reaction mixture was allowed to warm to $25{ }^{\circ} \mathrm{C}$ over $2 \mathrm{~h}$, then quenched with saturated aqueous $\mathrm{NaHCO}_{3}(10 \mathrm{~mL})$.

The resulting biphasic mixture was extracted with $\mathrm{Et}_{2} \mathrm{O}(3 \times 7 \mathrm{~mL})$ and the combined organic layers were dried $\left(\mathrm{Na}_{2} \mathrm{SO}_{4}\right)$, concentrated, and then purified by flash column chromatography (silica gel, EtOAc:hexanes 3:1) to give enone $136(0.78 \mathrm{~g}, 2.77 \mathrm{mmol}, 90 \%)$ as a colorless oil. 136: $R_{\mathrm{f}}=0.68$ (silica gel, EtOAc:hexanes 1:4); $[\alpha]_{\mathrm{D}}^{25}=+2.2\left(\mathrm{CHCl}_{3}, c=0.90\right)$; IR (film) $v_{\max }=3463,2929,2856,1422$, $1079 \mathrm{~cm}^{-1}$; ${ }^{1} \mathrm{H}$ NMR (500 MHz, $\left.\mathrm{CDCl}_{3}\right): \delta=6.22-6.18$ (m, $\left.1 \mathrm{H}\right), 6.12-6.08(\mathrm{~m}, 1 \mathrm{H}), 4.36(\mathrm{~m}, 2 \mathrm{H})$, 3.38-3.30 (m, 1 H), 2.96 (dq, $J=14.0,3.0$ Hz, 1 H), 2.89-2.83 (m, 4 H), 2.14-2.04 (m, 2 H), 1.89-1.79 (m, 2 H), 1.36-1.28 (m, 1 H), 1.10 (d, J = 7.0 Hz, 3 H), 1.06 (d, J = 7.0 Hz, 3 H), 0.92 (s, 9 H), 0.08 (s, $6 \mathrm{H}) ;{ }^{13} \mathrm{C}$ NMR (125 MHz, $\left.\mathrm{CDCl}_{3}\right): \delta=204.9,146.9,123.0,61.4,55.4,38.2,37.8,36.1,31.1,30.8$, 26.3, 25.9, 18.32, 18.28, 17.2, -5.43, -5.44; HR-MS [Matrix-assisted laser desorption / ionization (MALDI)]: calcd for $\mathrm{C}_{19} \mathrm{H}_{36} \mathrm{O}_{2} \mathrm{~S}_{2} \mathrm{SiNa}^{+}\left[\mathrm{M}+\mathrm{Na}^{+}\right]$: 411.1818, found 411.1820.

(S)-Allylic Alcohol 137. To a solution of enone 136 (146 mg, $376 \mu \mathrm{mol}, 1.0$ equiv) in toluene (8 mL) at $-78{ }^{\circ} \mathrm{C}$ was added Red-Al ${ }^{\circledR}(1.0 \mathrm{~mL}, 65 \%$ wt. in toluene, $940 \mu \mathrm{mol} 2.5$ equiv). Stirring was continued at

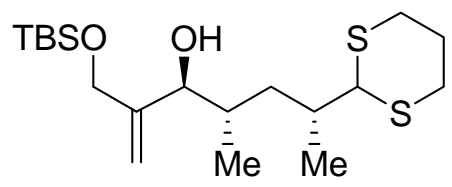

137 $-78{ }^{\circ} \mathrm{C}$ for $1 \mathrm{~h}$, then the reaction was quenched with saturated aqueous $\mathrm{NH}_{4} \mathrm{Cl}(50 \mathrm{~mL})$, saturated aqueous sodium, potassium tartrate $(50 \mathrm{~mL})$, and water $(50 \mathrm{~mL})$. The biphasic mixture was then extracted with $\mathrm{CH}_{2} \mathrm{Cl}_{2}(5 \times$ $35 \mathrm{~mL})$ and the combined organic extracts were dried $\left(\mathrm{Na}_{2} \mathrm{SO}_{4}\right)$, concentrated, and then purified by flash column chromatography (silica gel, EtOAc:hexanes 1:2) to afford a single isomer of (S)-allylic alcohol 137 (117 mg, $299 \mu \mathrm{mol}, 80 \%$ ) as a colorless oil. 137: $R_{\mathrm{f}}=0.46$ (silica gel, EtOAc:hexanes 1:4); $[\alpha]_{\mathrm{D}}{ }^{25}=$ -14.5 $\left(\mathrm{CHCl}_{3}, c=0.58\right)$; IR (film) $v_{\max }=3448,2927,2853,1420,1078 \mathrm{~cm}^{-1} ;{ }^{1} \mathrm{H}$ NMR $(500 \mathrm{MHz}$, $\left.\mathrm{CDCl}_{3}\right): \delta=5.14$ (d, $\left.J=1.5 \mathrm{~Hz}, 1 \mathrm{H}\right), 5.05$ (s, $\left.1 \mathrm{H}\right), 4.33$ (d, $\left.J=13.0 \mathrm{~Hz}, 1 \mathrm{H}\right), 4.19$ (d, $J=13.0 \mathrm{~Hz}, 1$ H), 4.18 (d, $J=3.5 \mathrm{~Hz}, 1 \mathrm{H}), 3.88$ (t, $J=6.5 \mathrm{~Hz}, 1 \mathrm{H}), 2.93$ (dt, $J=14.0,2.0 \mathrm{~Hz}, 1 \mathrm{H}), 2.87-2.81$ (m, 3 
H), 2.41 (d, $J=5.5 \mathrm{~Hz}, 1 \mathrm{H}), 2.14-1.98$ (m, $3 \mathrm{H}), 1.85-1.79$ (m, $2 \mathrm{H}), 1.12$ (d, J = $7.0 \mathrm{~Hz}, 3 \mathrm{H}), 1.04$ (ddd, $J=14.0,9.5,5.5 \mathrm{~Hz}, 1 \mathrm{H}), 0.92$ (s, $9 \mathrm{H}), 0.88$ (d, $J=7.0 \mathrm{~Hz}, 3 \mathrm{H}), 0.10(\mathrm{~s}, 6 \mathrm{H}) ;{ }^{13} \mathrm{C}$ NMR (125 $\left.\mathrm{MHz}, \mathrm{CDCl}_{3}\right): \delta=148.3,112.7,79.8,64.7,54.5,37.0,36.3,34.6,31.4,30.8,26.5,25.9,18.3,18.2$, 17.0, -5.40, -5.43; HR-MS [Matrix-assisted laser desorption / ionization (MALDI)]: calcd for $\mathrm{C}_{19} \mathrm{H}_{38} \mathrm{O}_{2} \mathrm{~S}_{2} \mathrm{SiNa}^{+}\left[\mathrm{M}+\mathrm{Na}^{+}\right]:$413.1975, found 413.1966.

Diol 138. To a solution of (S)-allylic alcohol 137 (30.0 mg, $0.077 \mathrm{mmol}, 1.0$ equiv) in THF (1.0 mL) at $0{ }^{\circ} \mathrm{C}$ was added TBAF (0.12 mL, $1.0 \mathrm{M}$ in THF, $0.12 \mathrm{mmol}, 1.5$ equiv). The reaction mixture was

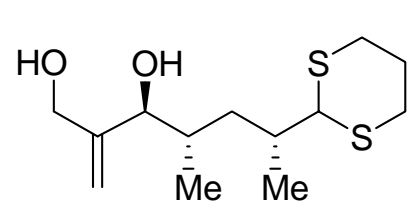

138 warmed to $25{ }^{\circ} \mathrm{C}$ over $1 \mathrm{~h}$ and then quenched with saturated aqueous $\mathrm{NH}_{4} \mathrm{Cl}(2$ $\mathrm{mL})$ and extracted with EtOAc $(3 \times 3 \mathrm{~mL})$. The combined organic extracts were dried $\left(\mathrm{Na}_{2} \mathrm{SO}_{4}\right)$, concentrated, and then purified by flash column chromatography (silica gel, EtOAc:hexanes 2:1) to afford diol 138 (21 mg, $0.076 \mathrm{mmol}, 99 \%)$ as a colorless oil. 138: $R_{f}=0.33$ (silica gel, EtOAc: hexanes $\left.1: 1\right) ;[\alpha]_{\mathrm{D}}{ }^{25}=+32.0(c=$ 2.90, $\left.\mathrm{CHCl}_{3}\right)$; IR (film): $v_{\max }=3416,2950,2928,1651,1455,1276,1025 \mathrm{~cm}^{-1} ;{ }^{1} \mathrm{H} \mathrm{NMR}(600 \mathrm{MHz}$, $\left.\mathrm{CDCl}_{3}\right): \delta=5.21(\mathrm{~s}, 1 \mathrm{H}), 5.12(\mathrm{~s}, 1 \mathrm{H}), 4.28(\mathrm{~d}, J=13.0 \mathrm{~Hz}, 1 \mathrm{H}), 4.20(\mathrm{~d}, J=3.5 \mathrm{~Hz}, 1 \mathrm{H}), 4.15(\mathrm{~d}, J=$ $13.0 \mathrm{~Hz}, 1 \mathrm{H}), 2.93$ (dt, $J=14.0,2.0 \mathrm{~Hz}, 1 \mathrm{H}), 2.84$ (dd, $J=8.3,3.1 \mathrm{~Hz}, 3 \mathrm{H}), 2.10$ (br s, $3 \mathrm{H}$ ), 2.08-2.03 (m, 1 H), 1.93 (ddd, $J=13.0,9.0,3.5$ Hz, 1 H), 1.87-1.80 (m, 2 H), 1.10 (d, J = 6.6 Hz, 3 H), 1.05 (ddd, $J=14.0,9.0,5.5 \mathrm{~Hz}, 1 \mathrm{H}), 0.90$ (d, $J=7.0 \mathrm{~Hz}, 3 \mathrm{H}) ;{ }^{13} \mathrm{C}$ NMR $\left(150 \mathrm{MHz}, \mathrm{CDCl}_{3}\right): \delta=148.6$, 113.9, 79.8, 64.3, 54.3, 36.4, 36.1, 34.3, 31.2, 30.7, 26.3, 18.3, 17.1; HR-MS [Matrix-assisted laser desorption / ionization (MALDI)]: calcd for $\mathrm{C}_{13} \mathrm{H}_{24} \mathrm{O}_{2} \mathrm{~S}_{2}\left[\mathrm{M}+\mathrm{Na}^{+}\right]$: 299.1110, found 299.1109.

Silylene 100. To a solution of diol $138\left(0.199 \mathrm{~g}, 0.721 \mathrm{mmol}, 1.0\right.$ equiv) in $\mathrm{CH}_{2} \mathrm{Cl}_{2}(5 \mathrm{~mL})$ at $-30{ }^{\circ} \mathrm{C}$ was added 2,6-lutidine (0.34 $\mathrm{mL}, 2.88 \mathrm{mmol}, 4.0$ equiv $)$ followed by di-tert-butylsilyl<smiles>C=C1CO[Si](C(C)(C)C)(C(C)(C)C)O[C@H]1[C@@H](C)C[C@H](C)C1SCCCS1</smiles>
bis(trifluoromethanesulfonate) $(0.25 \mathrm{~mL}, 1.15 \mathrm{mmol}, 1.6$ equiv). The reaction mixture was stirred at $-30{ }^{\circ} \mathrm{C}$ for $1 \mathrm{~h}$ and then quenched with saturated aqueous $\mathrm{NH}_{4} \mathrm{Cl}(5 \mathrm{~mL})$. The biphasic mixture was extracted with $\mathrm{CH}_{2} \mathrm{Cl}_{2}(3 \times$ $5 \mathrm{~mL})$ and the combined organic layers were dried $\left(\mathrm{Na}_{2} \mathrm{SO}_{4}\right)$, concentrated, 
and purified by flash column chromatography (silica gel, EtOAc:hexanes 1:9) to afford silylene 100 (0.251 g, $0.606 \mathrm{mmol}, 84 \%)$ as a clear solid. 100: $R_{f}=0.20\left(\right.$ EtOAc:hexanes 1:50); $[\alpha]_{\mathrm{D}}^{25}=+76.1(c=$ 1.4, $\mathrm{CHCl}_{3}$ ); IR (film): $v_{\max }=2930,2856,1632,1470,1085 \mathrm{~cm}^{-1} ;{ }^{1} \mathrm{H} \mathrm{NMR}\left(500 \mathrm{MHz}, \mathrm{CDCl}_{3}\right) \delta=5.10$ (s, 1 H), 4.91 (s, 1 H), 4.53 (d, $J=11.0 \mathrm{~Hz}, 1 \mathrm{H}$ ), 4.17 (d, $J=15.0 \mathrm{~Hz}, 1 \mathrm{H}), 4.16$ (d, $J=11.0 \mathrm{~Hz}, 1 \mathrm{H}$ ), 2.97-2.91 (m, 1 H), 2.86-2.80 (m, 3 H), 2.18 (ddd, $J=12.0,8.5,4.0$ Hz, 1 H), 2.12-2.05 (m, 2 H), 1.86-1.77 (m, 3 H), 1.13 (d, $J=7.0$ Hz, 3 H), 1.07 (ddd, $J=15.0,10.0,5.5$ Hz, 1 H), 1.04 (s, 9 H), 0.98 (s, $9 \mathrm{H}$ ), 0.84 (d, $J=7.0 \mathrm{~Hz}, 3 \mathrm{H}) ;{ }^{13} \mathrm{C}$ NMR $\left(125 \mathrm{MHz}, \mathrm{CDCl}_{3}\right) \delta=146.0,114.0,81.5$, 67.4, 54.5, 37.0, 36.5, 31.4, 30.6, 27.3, 26.9, 26.4, 21.4, 20.9, 18.2, 16.3; HR-MS [Matrix-assisted laser desorption / ionization (MALDI)]: calcd for $\mathrm{C}_{21} \mathrm{H}_{40} \mathrm{O}_{2} \mathrm{~S}_{2} \mathrm{SiNa}\left[\mathrm{M}+\mathrm{Na}^{+}\right]$: 439.2136, found 439.2155 .

\section{References:}

(1) Saito, S.; Ishikawa, T.; Kuroda, A.; Koga, K.; Moriwake, T. Tetrahedron 1992, 48, 4067.

(2) Schoning, K.-U.; Hayashi, R. K.; Powell, D. R.; Kirshning, A. Tetrahedron: Asymmetry 1999, 10, 817.

(3) Allingham, M. T.; Howard-Jones, A.; Murphy, P. J.; Thomas, D. A.; Caulkett, P. W. R. Tetrahedron Lett. 2003, 44, 8677.

(4) Ghosh, A. K.; Cappiello, J.; Shin, D. Tetrahedron Lett. 1998, 39, 4651.

(5) Collum, D. B.; McDonald, J. H.; Still, W. C. J. Am. Chem. Soc. 1980, 102, 2118.

(6) Somfai, P.; Olsson, R. Tetrahedron 1993, 49, 6645.

(7) Racherla, U. S.; Brown, H. C. J. Org. Chem. 1991, 56, 401.

(8) Rosowsky, A.; Wright, J. E. J. Org. Chem. 1983, 48, 1539. 


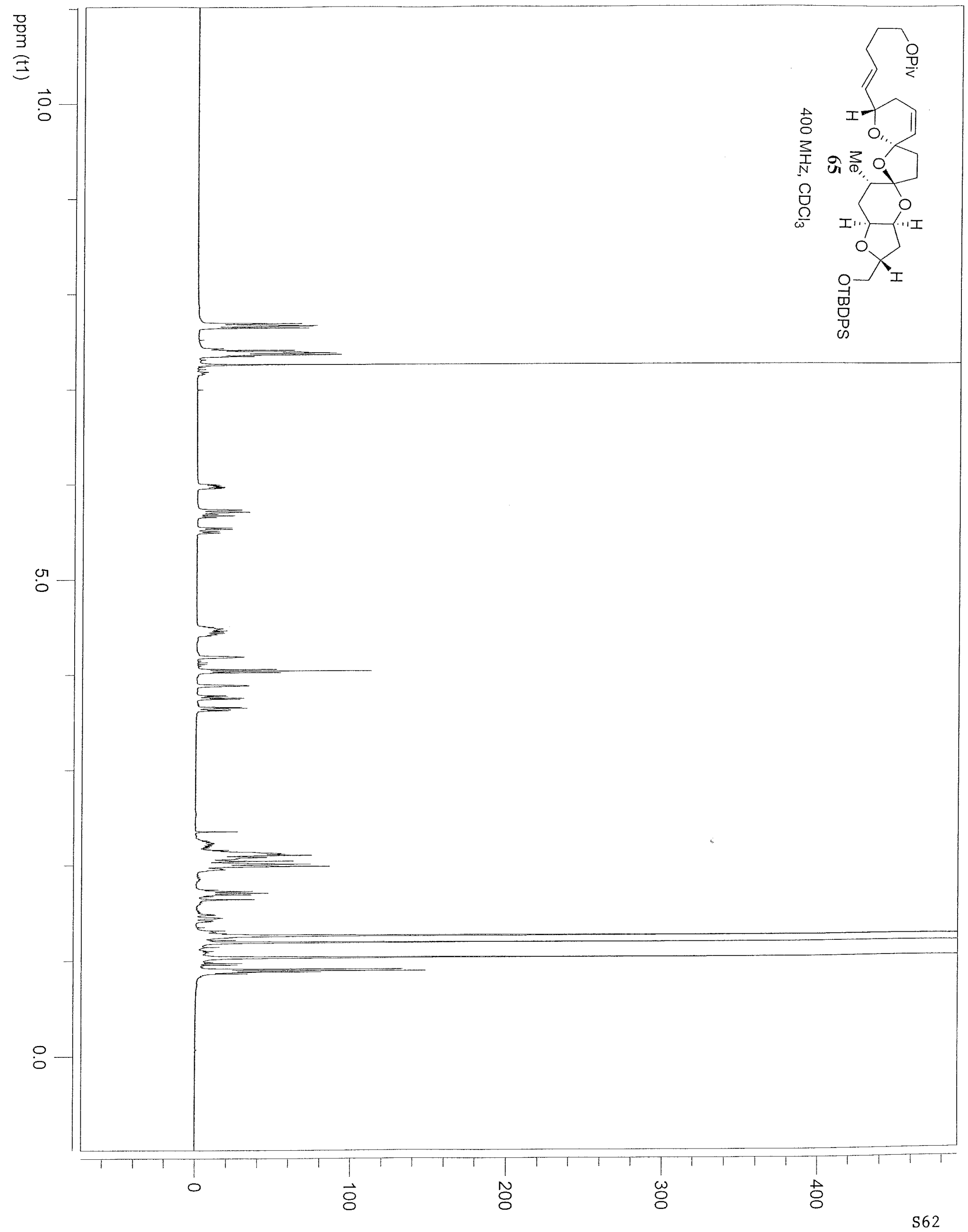




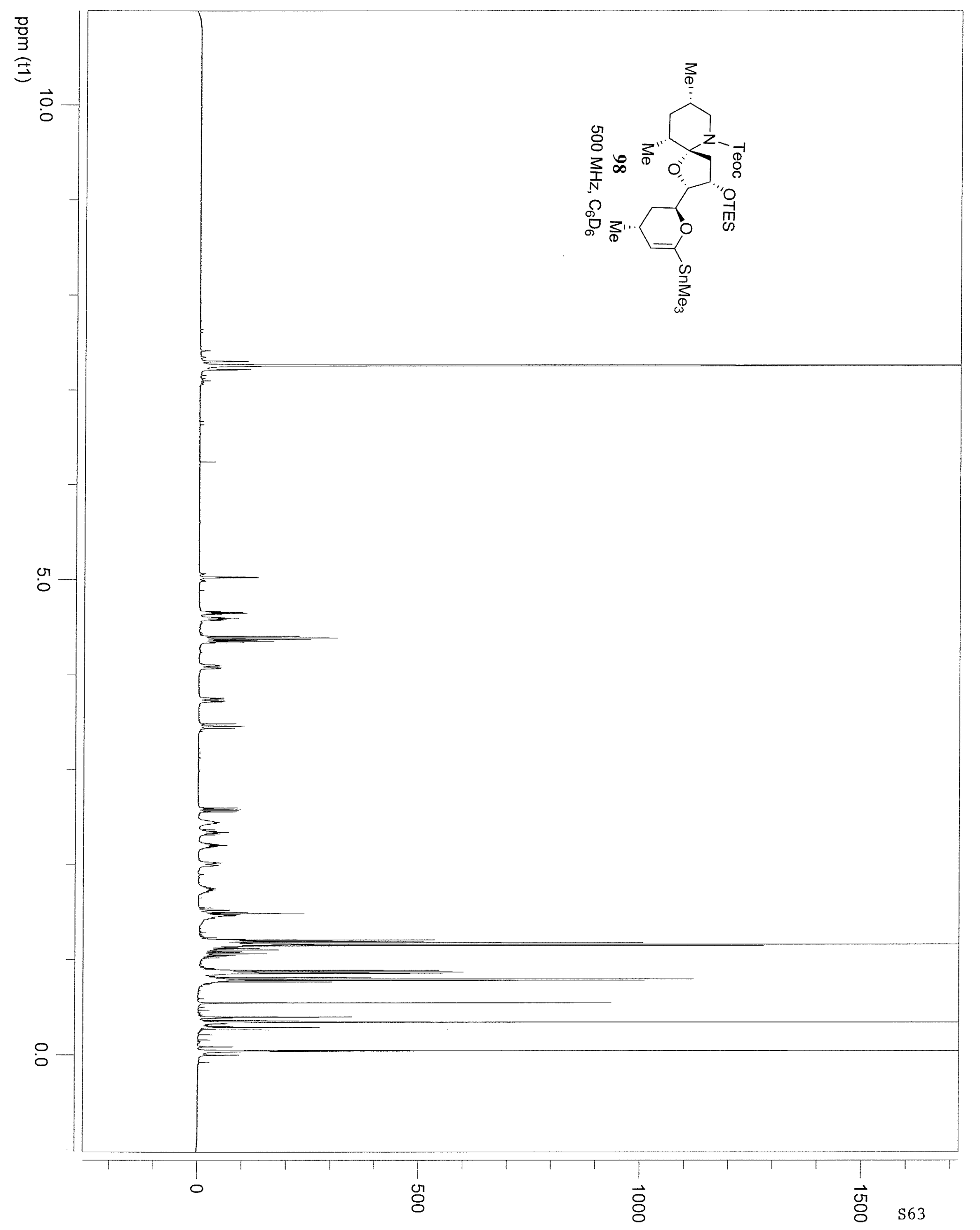




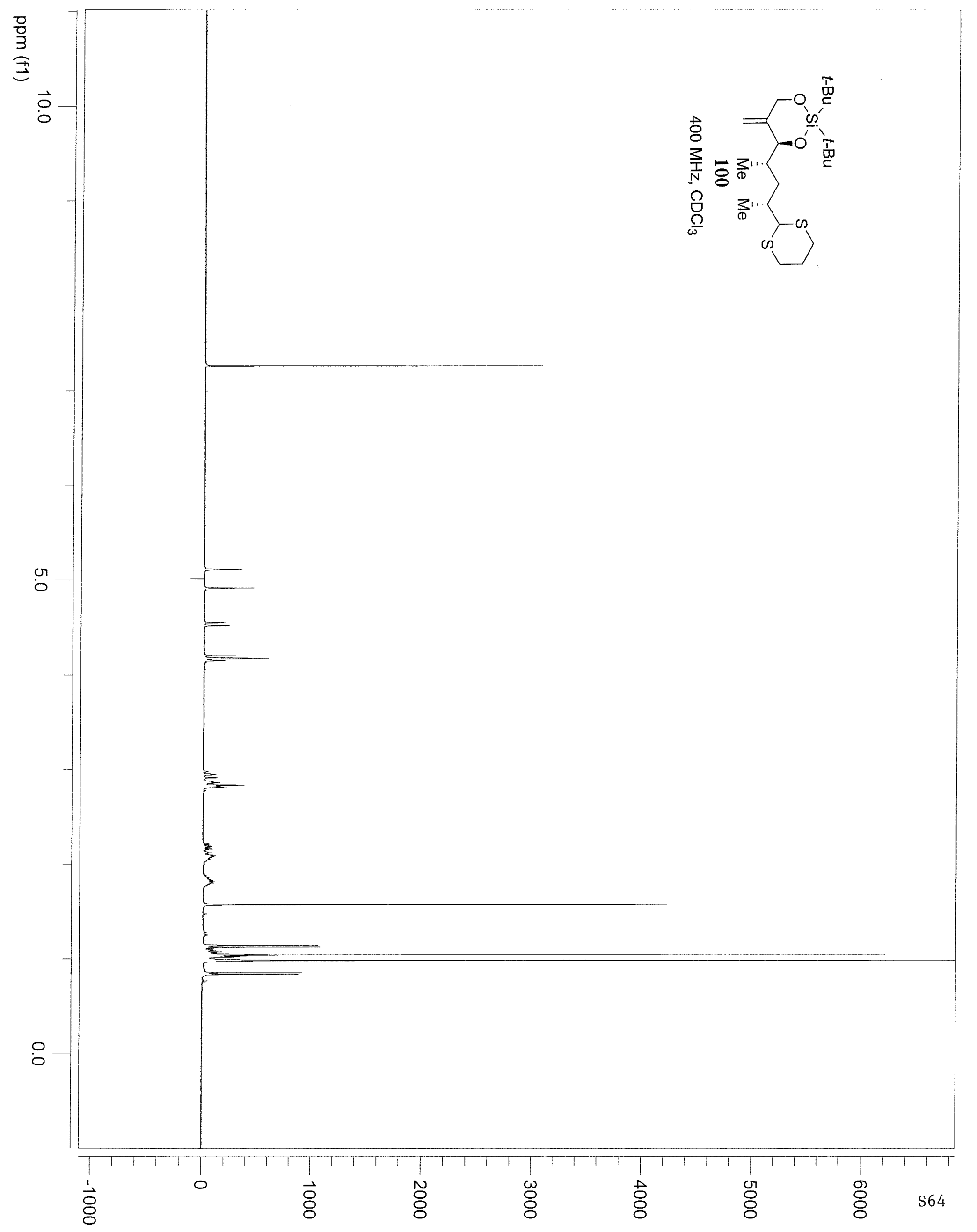

